



\title{
Análise preditiva de desempenho de workflows usando Teoria do Campo Médio
}

\author{
Waldir Edison Farfan Caro \\ DISSERTAÇÃO DE MESTRADO \\ INSTITUTO DE MATEMÁTICA E ESTATÍSTICA \\ DA \\ UNIVERSIDADE DE SÃo PAULO \\ Programa: Ciência da Computação \\ Orientador: Profa. Dra. Kelly Rosa Braghetto
}

Durante o desenvolvimento deste trabalho o autor recebeu auxílio financeiro da CAPES 


\section{Análise preditiva de desempenho de workflows usando Teoria do Campo Médio}

Esta versão da dissertação/tese contém as correções e alterações sugeridas pela Comissão Julgadora durante a defesa da versão original do trabalho, realizada em 17/04/2017. Uma cópia da versão original está disponível no Instituto de Matemática e Estatística da Universidade de São Paulo.

Comissão Julgadora:

- Prof $^{a}$. Dra ${ }^{a}$ Kelly Rosa Braghetto (orientadora) - IME-USP

- Prof. Dr. Marco Dimas Gubitoso - IME-USP

- Prof. Dr. Afonso Henrique Corrêa de Sales - PUC-RS 


\section{Agradecimentos}

Agradeço a Deus pela oportunidade de realizar este trabalho fora da minha cidade, e tudo o que aconteceu ao longo desse tempo no Brasil. Minha família que sempre me apoia e me impulsiona a melhorar, em especial minha mãe Margarita que sem dúvidas tem sido uma inspiração de superação para mim e meus irmãos Daniel, Esthefanny e Piero, aos meus tios e avós. Da mesma forma à minha família espiritual que me amparou em tudo momento.

Sou muito grato com a minha orientadora, a professora Kelly Rosa Braghetto, pela dedicação, orientação e a paciência dela no trabalho da pesquisa científica.

Agradeço aos professores Marco Gubitoso (IME-USP), Alfredo Goldman (IME-USP) e Afonso Corrêa de Sales (PUC-RS), membros da banca de qualificação e defesa, pelas observações e conselhos para fazer um melhor trabalho. Assim como ao professor Jean-Marc Vincent, do Laboratoire de Informatique de Grenoble (LIG), que auxiliou e forneceu conhecimento relevante para o desenvolvimento deste trabalho.

Finalmente, agradeço ao IME-USP pela oportunidade e experiência acadêmica, ao CAPES pelo apoio financeiro, e à UNSAAC, minha primeira universidade que me incentivou o gosto pelas ciências da computação. Por cada pessoa que conheci aqui, Hans, Grover, Miguel, Mariela, Edu, Lucy, Anderson, Fernando, Paulo, Sabrina, Juan e outros com os quais compartilhei experiências acadêmicas e cotidianas. 


\section{Resumo}

FARFAN CARO, WALDIR E. Análise preditiva de desempenho de workflows usando Teoria do Campo Médio. 2017. 98 p. Tese (Mestrado) - Instituto de Matemática e Estatística, Universidade de São Paulo, São Paulo, 2017.

Os processos de negócio desempenham um papel muito importante na indústria, principalmente pela evolução das tecnologias da informação. As plataformas de computação em nuvem, por exemplo, com a alocação de recursos computacionais sob demanda, possibilitam a execução de processos altamente requisitados. Para tanto, é necessário definir o ambiente de execução dos processos de tal modo que os recursos sejam utilizados de forma ótima e seja garantida a correta funcionalidade do processo. Nesse contexto, diferentes métodos já foram propostos para modelar os processos de negócio e analisar suas propriedades quantitativas e qualitativas. Há, contudo, vários desafios que podem restringir a aplicação desses métodos, especialmente para processos com alta demanda (como os workflows de numerosas instâncias) e que dependem de recursos limitados.

A análise de desempenho de workflows de numerosas instâncias via modelagem analítica é o objeto de estudo deste trabalho. Geralmente, para a realização desse tipo de análise usa-se modelos matemáticos baseados em técnicas Markovianas (sistemas estocásticos), que sofrem do problema da explosão do espaço de estados. Entretanto, a Teoria do Campo Médio indica que o comportamento de um sistema estocástico, sob certas condições, pode ser aproximado por o de um sistema determinístico, evitando a explosão do espaço de estados.

Neste trabalho usamos tal estratégia e, com base na definição formal de aproximação determinística e suas condições de existência, elaboramos um método para representar os workflows, e seus recursos, como equações diferenciais ordinárias, que descrevem um sistema determinístico. Uma vez definida a aproximação determinística, realizamos a análise de desempenho no modelo determinístico, verificando que os resultados obtidos são uma boa aproximação para a solução estocástica.

Palavras-chave: workflows de negócio, modelagem analítica, análise de desempenho, aproximação de campo médio. 


\section{Abstract}

FARFAN CARO, WALDIR E. Predictive performance analysis of workflows using Mean Field Theory. 2017. 98 p. Thesis (Master of Science) - Institute of Mathematic and Statistic, University of São Paulo, São Paulo, 2017.

Business processes play a very important role in the industry, especially by the evolution of information technologies. Cloud computing platforms, for example, with the allocation of on-demand computing resources enable the execution of highly requested processes.

Therefore, it is necessary to define the execution environment of the processes in such a way that the resources are used optimally and the correct functionality of the process is guaranteed.

In this context, different methods have already been proposed to model business processes and analyze their quantitative and qualitative properties. There are, however, a number of challenges that may restrict the application of these methods, especially for high-demanded processes (such as workflows of numerous instances) and that rely on resources that are limited.

The analysis of the performance of workflows of numerous instances through analytical modeling is the object of study of this work. Generally, for the accomplishment of this type of analysis, mathematical models based on Markovian techniques (stochastic systems) are used, which suffer the problem of the state space explosion. However, the Mean Field Theory, indicates that the behavior of a stochastic system, under certain conditions, can be approximated by that of a deterministic system, avoiding the explosion of the state space.

In this work we use such a strategy, based on the formal definition of deterministic approximation and its conditions of existence, we elaborate a method to represent the workflows, and their resources, as ordinary differential equations, which describe a deterministic system. Once the deterministic approximation has been defined, we perform the performance analysis in the deterministic model, verifying that the obtained results are a good approximation for the stochastic solution.

Keywords: business workflows, analytical modeling, performance analysis, mean field approximation. 


\section{Sumário}

$\begin{array}{ll}\text { Lista de Abreviaturas } & \text { ix }\end{array}$

Lista de Figuras $\quad$ xi

Lista de Tabelas $\quad$ xiii

1 Introdução $\quad 1$

1.1 Contexto e Motivação do Trabalho . . . . . . . . . . . . . . . . . . 1

1.2 Descrição do Problema . . . . . . . . . . . . . . . . . . . . 2

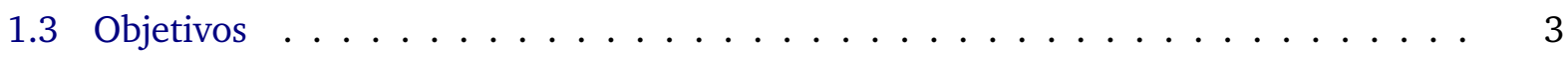

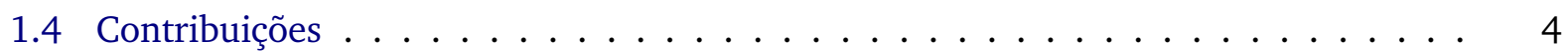

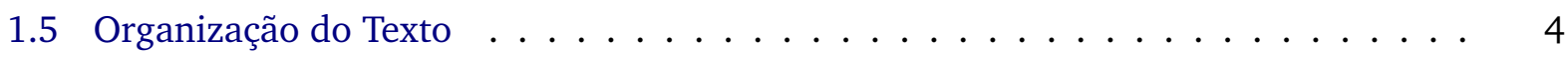

2 Conceitos $\quad 7$

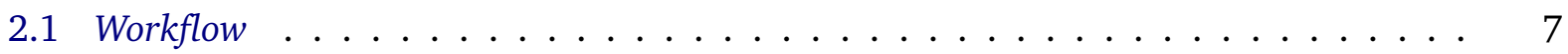

2.1.1 Fluxo de Controle $\times$ Fluxo de Dados . . . . . . . . . . . . . 8

2.1 .2 Modelagem de Workflows . . . . . . . . . . . . . . . 8

2.2 Business Process Model and Notation (BPMN) . . . . . . . . . . . . . . . . . . 10

2.3 Recursos no Workflow . . . . . . . . . . . . . . . . . . . . . . . 13

2.4 Análise de Sistemas Computacionais _ . . . . . . . . . . . . . . . . . 15

2.5 Seleção de Métricas de Desempenho . . . . . . . . . . . . . . . . . . . . 17

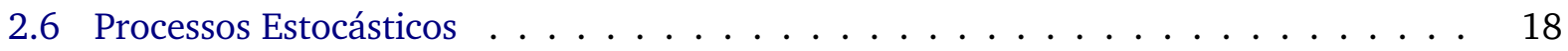

2.6.1 Cadeias de Markov . . . . . . . . . . . . . . . . . . . . . . . 19

2.6 .2 Análise de Regime Estacionário . . . . . . . . . . . . . . . . . 21

2.6 .3 Análise Transiente . . . . . . . . . . . . . . . . . . . . . . . . 21

2.7 Ferramentas para modelagem analítica . . . . . . . . . . . . . . . . . 21

2.7 .1 Redes de Petri . . . . . . . . . . . . . . . . . . . . . 22

3 Modelagem markoviana de workflows de negócio de numerosas instâncias $\quad 27$

3.1 Modelagem Markoviana de Estruturas Básicas de Workflows . . . . . . . . . . . . 27

3.1.1 Mapeamento de uma Atividade . . . . . . . . . . . . . . . . . . 27

3.1.2 Mapeamento de uma Sequência de Atividades . . . . . . . . . . . . . . . . . 29

3.1 .3 Mapeamento de uma Escolha . . . . . . . . . . . . . . . . . . . . . . 29

3.1 .4 Mapeamento do Paralelismo . . . . . . . . . . . . . . . . . . . 30

3.2 Análise de Desempenho de Modelos Markovianos . . . . . . . . . . . . . . . . . 32

3.3 Explosão do Espaço de Estados . . . . . . . . . . . . . . . . . . . . . . 39 
4 Geração de espaços de estados contínuos para modelos de workflows 41

4.1 Teoria do Campo Médio (Mean Field Theory) . . . . . . . . . . . . . . . . . . . . . 41

4.1 .1 Aproximação determinística de CMTDs síncronas . . . . . . . . . . . . . . 42

4.1 .2 Aproximação determinística de CMTCs . . . . . . . . . . . . . . . . . . . . . 44

4.2 Aproximação Determinística para Workflows . . . . . . . . . . . . . . . . . 48

4.2 .1 Modelo de CMTCPs para Workflows . . . . . . . . . . . . . . . . . . . 48

4.2 .2 SDTC do modelo de workflow . . . . . . . . . . . . . . . . 54

5 Extração de métricas de desempenho a partir de ODEs $\quad 57$

5.1 Índices de Desempenho . . . . . . . . . . . . . . . . . . . . . . 58

5.1 .1 Número de Unidades dos Recursos em Uso . . . . . . . . . . . . . . . . . . 59

5.1 .2 Tamanho da Fila dos Recursos . . . . . . . . . . . . . . . . . . . . . 59

5.1 .3 Rendimento de Recursos . . . . . . . . . . . . . . . . . . . . . . . . 59

5.1 .4 Rendimento de Atividades . . . . . . . . . . . . . . . . . . . . . . 59

5.1 .5 Rendimento do Sistema . . . . . . . . . . . . . . . . . . . 60

5.2 Alguns Resultados de Soluções Estocásticas e Determinísticas para Workflows . . . . 60

5.3 Um Exemplo Mais Complexo: Workflow de Controle de Pedidos . . . . . . . . . . 70

5.4 Considerações sobre os resultados . . . . . . . . . . . . . . . . . . 73

6 Conclusões $\quad 75$

6.1 Considerações Finais . . . . . . . . . . . . . . . . . . 75

6.2 Sugestões para Pesquisas Futuras . . . . . . . . . . . . . . . . . 76

$\begin{array}{ll}\text { Referências Bibliográficas } & 79\end{array}$ 


\section{Lista de Abreviaturas}

BPMN Modelo e Notação de Processos de Negócio (Business Process Model and Notation)

CMTC Cadeia de Markov de Tempo Contínuo (Continuous Time Markov Chain)

CMTD Cadeia de Markov de Tempo Discreto (Discrete Time Markov Chain)

CMTCP Cadeia de Markov de Tempo Contínuo de População (Population CTMC)

ODE Equação Diferencial Ordinária (Ordinary Differential Equation)

RPE $\quad$ Rede de Petri Estocástica (Stochastic Petri Net)

RPEG Rede de Petri Estocástica Generalizada (Generalized Stochastic Petri Net)

SDTC Sistema Determinístico de Tempo Contínuo (Continuous Time Deterministic System)

SDTD Sistema Determinístico de Tempo Discreto (Discrete Time Deterministic System)

TCM Teoria do Campo Médio (Mean Field Theory) 


\section{Lista de Figuras}

2.1 Modelo de workflow bem-definido em notação BPMN. . . . . . . . . . . . . . . . 13

2.2 Modelo simples de uma Rede de Petri, onde os lugares são P1, P2 e P3 e as transições são T1, T2, T3 e T4. A marca da Rede de Petri é $\{2,1,0\} \ldots \ldots$. . . . . . . . . . . . . . . . . . . . . . . . . . . . .

2.3 Rede de Petri reversível _. . . . . . . . . . . . . . . . . . . . 25

2.4 Grafo de alcançabilidade da Rede de Petri em $2.3 \ldots \ldots$. . . . . . . . . . . . 26

3.1 Mapeamento em RPGE de uma atividade $A$ com requisito $P_{A}=\left(\left(\left[R_{1}, a\right] \wedge\left[R_{2}, b\right]\right) \vee\right.$

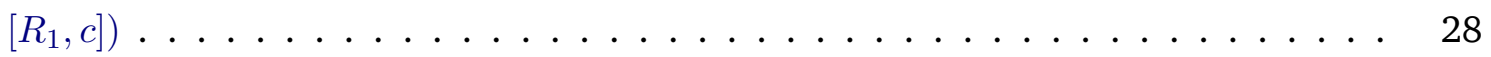

3.2 Modelo BPMN com estrutura sequencial de atividades. . . . . . . . . . . . . . . . . . . . . . . . . 29

3.3 RPEG da estrutura sequencial mostrada na Figura 3.2 . . . . . . . . . . . . . . . . . 29

3.4 Modelo BPMN com estrutura de escolha . . . . . . . . . . . . . . . . . . . . . . . . . . . . . . .

3.5 RPEG da estrutura de escolha mostrada na Figura $3.4 \ldots \ldots \ldots$

3.6 Modelo BPMN com estrutura paralela . . . . . . . . . . . . . . . . . 31

3.7 RPEG modelando a estrutura paralela mostrada na Figura 3.6 . . . . . . . . . . . 31

3.8 Modelo de workflow que apresenta a combinação das três estruturas básicas: sequên-

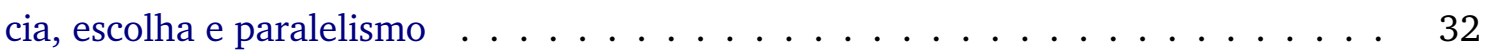

3.9 Modelagem por meio de RPEG do modelo de workflow mostrado na Figura 3.8 . . . 32

3.10 Grafo de alcançabilidade da RPEG mostrada na Figura 3.3 quando existe só uma ficha em $P_{0}$ (número de instâncias). . . . . . . . . . . . . . .

3.11 Evolução no tempo, das quantidades médias de fichas nos lugares da RPEG mostrada na Figura 3.3, calculados de acordo com o grafo de alcançabilidade da Figura 3.10.

3.12 Trajetórias dos valores médios nos lugares na RPEG da Figura 3.3, quando mudamos o número de fichas inicial para $I=3 \ldots \ldots \ldots \ldots$

3.13 Trajetórias das quantidades médias de fichas nos lugares da RPEG da Figura 3.5, quando $I=5$ e $N R_{1}=10 \ldots \ldots \ldots \ldots \ldots \ldots \ldots$

3.14 Trajetórias das quantidades médias de fichas nos lugares da RPEG da Figura 3.5, quando $I=10$ e $N R_{1}=10 \ldots \ldots \ldots \ldots \ldots \ldots \ldots \ldots \ldots \ldots \ldots \ldots \ldots \ldots$

3.15 Trajetórias dos valores médios nos lugares da RPEG da Figura 3.5, quando $I=15$ e $N R_{1}=10 \ldots \ldots \ldots \ldots \ldots \ldots \ldots \ldots \ldots \ldots \ldots \ldots \ldots \ldots \ldots \ldots \ldots \ldots$

3.16 Trajetórias dos valores médios nos lugares da RPEG da Figura 3.7, quando $I=5$ e $N R_{1}=10 \ldots \ldots \ldots \ldots \ldots \ldots \ldots \ldots \ldots \ldots \ldots \ldots \ldots \ldots \ldots \ldots \ldots \ldots$

3.17 Trajetórias dos valores médios nos lugares da RPEG da Figura 3.7, quando $I=10 \mathrm{e}$ $N R_{1}=10 \ldots \ldots \ldots \ldots \ldots \ldots \ldots \ldots \ldots \ldots \ldots \ldots \ldots \ldots \ldots \ldots \ldots$

3.18 Trajetórias dos valores médios nos lugares da RPEG da Figura 3.7, quando $I=15$ e

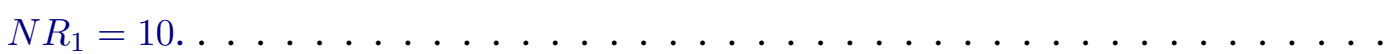

4.1 Modelo de workflow bem definido . . . . . . . . . . . . . . . . 
4.2 Estados e transições gerados no mapeamento da atividade $A . \ldots \ldots$. . . . .

4.3 Representação de estados e transições da permutação $\sigma=\left(\sigma\left(B_{1}\right), \ldots, \sigma\left(B_{x}\right)\right)$ (para o mapeamento do paralelismo) . . . . . . . . . . . . . . . .

4.4 Estados e transições geradas para estruturas de escolha. Em (a) está o XOR-split; e (b) representa o XOR-join. . . . . . . . . . . . . . . . . . . .

4.5 Estados (círculos) e transições (flechas), que representam a execução de uma instância do modelo de workflow da Figura 4.1. . . . . . . . . . . . . . . . . .

4.6 Estados (em circulo) e transições (flechas) finais, depois de remover as transições com rótulo null do mapeamento na Figura 4.5 . . . . . . . . . . . . . . . .

5.1 Workflow com estrutura sequencial de atividades . . . . . . . . . . . .

5.2 Comparação dos comportamentos estocástico e determinístico do workflow da Figura 5.1. Em (b), das ODEs calculamos $X_{R 1}(t)=N R_{1}-\left(X_{P 1}(t)+X_{P 3}(t)\right) \ldots \ldots \quad 58$

5.3 Modelo BPMN com estrutura de escolha . . . . . . . . . . . . . . . . . . 61

5.4 RPEG da estrutura de escolha mostrada na Figura $5.3 \ldots \ldots$. . . . . . . . . . . . 61

5.5 Comparação dos comportamentos estocástico e determinístico do workflow da Figura 5.3 para $I=3$ e $N R_{1}=2$. Em (b) temos que $X_{R 1}(t)=N R_{1}-\left(X_{P 2}(t)+X_{P 4}(t)\right) .62$

5.6 Comparação dos comportamentos estocástico e determinístico similar à Figura 5.5, quando $I=30$ e $N R_{1}=20 \ldots \ldots \ldots \ldots \ldots \ldots \ldots \ldots \ldots \ldots$

5.7 Modelo BPMN com estrutura paralela . . . . . . . . . . . . . . . . . . 64

5.8 RPEG modelando a estrutura paralela mostrada na Figura $5.7 \ldots \ldots$. . . . . . . . . 64

5.9 Comparação dos comportamentos estocástico e determinístico do workflow da Figura 5.7. Em (b) temos que $X_{R 1}(t)=N R_{1}-\left(X_{P 1}(t)+X_{P 3}(t)+X_{P 4}(t)+X_{P 6}(t)\right)$.

5.10 Comparação dos comportamentos estocástico e determinístico similar à Figura 5.9, quando $I=15$ e $N R_{1}=10 \ldots \ldots \ldots \ldots \ldots \ldots \ldots \ldots$

5.11 Modelo BPMN composto de estruturas básicas. . . . . . . . . . . . . . . . . . 67

5.12 RPEG modelando o modelo de workflow mostrado na Figura 5.11. . . . . . . . . . . 67

5.13 Comparação dos comportamentos estocástico e determinístico do workflow da Figura 5.11 para $I=5$ e $N R 1=5$. Em (b) temos que $X_{R 1}(t)=N R_{1}-\left(X_{P 2}(t)+\right.$ $\left.X_{P 4}(t)+X_{P 5}(t)+X_{P 7}(t)+X_{P 9}(t)+X_{P 11}(t)\right) \ldots \ldots \ldots \ldots \ldots$

5.14 Comparação dos comportamentos estocástico e determinístico do workflow da Figura 5.11 para $I=10$ e $N R 1=5$. Os valores finais são descritos na Tabela 5.6. . . 69

5.15 RPEG do um workflow de controle de pedidos (Extraído de [AD13]) . . . . . . . . . 71 


\section{Lista de Tabelas}

2.1 Elemento de fluxo do BPMN usados neste trabalho $\ldots \ldots \ldots \ldots$

3.1 Descrição dos componentes da RPEG na Figura 3.1 . . . . . . . . . . . . . . . . 28

3.2 Descrição dos componentes da RPEG na Figura 3.3 . . . . . . . . . . . . . . . . . . 29

3.3 Descrição dos componentes da RPEG na Figura 3.5 . . . . . . . . . . . . . . . . . 30

3.4 Descrição dos componentes da RPEG na Figura 3.7 . . . . . . . . . . . . . . . . . . 31

3.5 Tamanho do grafo de alcançabilidade da RPEG da Figura 3.3 (em termos do número de estados e transições) e custo computacional (em termos de tempo de processamento e consumo de memória) para obtê-lo, para diferentes configurações iniciais no número de instâncias (I) e de recursos $\left(N R_{1}\right) \ldots \ldots \ldots \ldots$. . . . . . 40

5.1 Valores das populações da Figura 5.2. Onde o erro porcentual foi calculado como

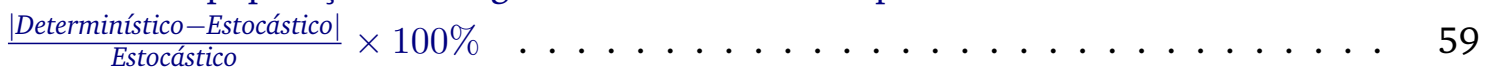

5.2 Valores das populações das trajetórias da Figura $5.5 \ldots$. . . . . . . . . . . . . 61

5.3 Valores das populações das trajetórias da Figura 5.6. . . . . . . . . . . . . . . . . 61

5.4 Valores das populações das trajetórias da Figura 5.9 . . . . . . . . . . . . . . 64

5.5 Valores das populações das trajetórias da Figura 5.10 . . . . . . . . . . . . 64

5.6 Distribuição das populações das soluções estocástica e determinística para o modelo de workflow da Figura 5.11 com 10 instâncias e 5 unidades de recurso ( $I=10$ e $N R_{1}=5$ ), cuja evolução está descrita na Figura 5.14 . . . . . . . . . . 67

5.7 Soluções estocástica e determinística para a RPEG descrita na Figura 5.15 . . . . 73 


\section{Capítulo 1}

\section{Introdução}

\subsection{Contexto e Motivação do Trabalho}

O vertiginoso crescimento de serviços computacionais oferecidos, assim como sua alta demanda, tem originado a denominada computação ubíqua, com a finalidade de tornar habitual a interação humano computador. Como consequência, o processamento computacional tem crescido muito nos últimos anos, oferecendo novos patamares para execução de processos. Muitas empresas oferecem centenas de serviços, principalmente na internet, que podem chegar a ser solicitados milhões de vezes em curtos períodos de tempo, exigindo recursos capazes de atender todas as demandas (de execução de processos), sem comprometer a qualidade de serviço.

Nesse cenário, onde os processos de negócio, ou serviços, têm se tornado mais complexos tanto na estrutura quanto na escala na execução, diversos desafios tem surgido com respeito à eficiência, confiabilidade, disponibilidade, os quais estão relacionados com propriedades quantitativas e qualitativas do processo. Assim, é indispensável realizar uma boa gestão dos processos, que além de prover melhor qualidade de serviço, pode reduzir o custo operacional do ambiente de execução. Por essa razão, mesmo em plataformas que disponibilizam recursos "quase infinitos", como as de Computação em Nuvem, é essencial otimizar o uso de recursos ao mesmo tempo em que se mantém os níveis de qualidade de serviço.

Os sistemas de workflows foram desenvolvidos para facilitar o gerenciamento dos processos de negócio [Hol95, WfM99]. Um workflow pode ser visto como uma ferramenta para a automação total ou parcial de um processo, que descreve um conjunto de atividades cuja ordem de execução é definida por regras de controle. As atividades, ao serem executadas, produzem um resultado referente ao objetivo do processo. Especificamente para processos de negócio chamamos sua automatização de workflows de negócio.

Os workflows são uma ferramenta útil não apenas para a automação dos processos, mas também para a otimização dos mesmos. Na análise de um workflow, procura-se investigar como as diferentes características do workflow - relacionadas à forma como suas atividades estão estruturadas e aos recursos dos quais elas dependem - impactam a sua confiabilidade e o seu tempo de execução, para assim determinar um ambiente computacional apropriado para sua execução. A análise também nos permite identificar problemas na estrutura do workflow resultantes de falhas de projeto ou de implementação, tais como estados de impasse (deadlocks) e gargalos. A análise de um workflow pode ser feita em diferentes momentos do seu ciclo de vida. Entretanto, é melhor realizar a análise nas primeiras etapas do desenvolvimento do workflow, pois geralmente problemas identificados em tempo de projeto são mais fáceis de serem resolvidos do que problemas em workflows já em implementação ou execução . 
Podemos destacar dois tipos principais de análise nos workflows: qualitativa e quantitativa. A análise qualitativa busca avaliar propriedades referentes ao comportamento do workflow (também conhecidas como propriedades funcionais), ou seja, determinar se ele realiza o que é esperado dele. Já a análise quantitativa procura avaliar caraterísticas estruturais (também chamadas de propriedades não-funcionais), como o consumo de recursos e a qualidade de serviço. Dentro da análise quantitativa identificamos a análise preditiva de desempenho, que visa avaliar o desempenho de um workflow antes mesmo deste ser implementado e executado. A avaliação de desempenho é definida por meio de métricas que medem diversas propriedades do sistema, como utilização de recursos, tempos de execução de atividades, rendimento do sistema, entre outros.

Existem diferentes técnicas que podem ser usadas para a análise de desempenho de workflows. Entretanto, a escolha da técnica mais apropriada depende de diversos fatores, dentre os quais podemos destacar o nível de desenvolvimento do sistema, o prazo para a obtenção e a precisão requerida dos resultados de análise, ou ainda o custo de se fazer a análise. Essas técnicas seguem três tipos de abordagem: medição, simulação e modelagem analítica [Jai91].

Neste trabalho o foco é a análise preditiva de desempenho de workflows via modelagem analítica, que é uma abordagem baseada em modelos matemáticos para o estudo de sistemas dinâmicos. Uma das técnicas matemáticas que tem proporcionado melhores resultados são os Processos Estocásticos, principalmente um subconjunto destes, denominado Processos de Markov [BW90, $\mathrm{R}^{+}$96]. Ferramentas baseadas em processos de Markov, como as Redes de Petri, foram desenvolvidas para facilitar a modelagem analítica, abstraindo os conceitos matemáticos subjacentes aos processos estocásticos. Apesar disso, as técnicas estocásticas têm se apresentado insuficientes para analisar processos complexos, devido ao problema da explosão do espaço de estados. Modelos estocásticos para processos de grande escala, como os descritos anteriormente, podem gerar uma quantidade ingente de estados, tornando o processamento computacional para analisar as medidas de desempenho impraticável (em termos de tempo e consumo de memória).

Dados workflows de negócio de numerosas instâncias, para processos com alta demanda, e tendo em vista a problemática das abordagens atuais, este trabalho busca propor uma nova abordagem de modelagem analítica para a análise de desempenho preditiva deste tipo de sistemas. Com essa finalidade, desenvolver-se-á uma metodologia matemática capaz de modelar o sistema e que permita o cálculo de medidas de desempenho em tempos e com recursos computacionais razoáveis.

\subsection{Descrição do Problema}

A análise preditiva de desempenho de workflows é uma tarefa desafiadora, ainda mais para processos com alta demanda. A maioria das técnicas usadas para estimar o desempenho destes sistemas estão baseadas em processos estocásticos, nos quais é conhecido o problema da explosão do espaço de estados. Esse problema se refere ao crescimento (exponencial) do espaço de estados em função do número de componentes do sistema. Com frequência, é irrealizável o cálculo da solução numérica desses modelos em tempo polinomial, o que inviabiliza o uso dessas técnicas na análise de sistemas de workflow com um grande número de instâncias.

Um exemplo de sistema com essas características é a execução concorrente de milhares de instâncias de um mesmo workflow em uma plataforma de computação em nuvem (como poderia ocorrer em uma aplicação de comércio eletrônico). Consequentemente, o principal problema a ser resolvido é como fazer a análise de desempenho preditiva do workflow, evitando o rápido 
crescimento do espaço de estados e o custo computacional que ele implica.

O triângulo de gerenciamento de projetos [Atk99] (também chamado de triângulo de restrições), é um modelo da área de gestão de projetos que procura medir a qualidade de um projeto sob três características: (i) ser bom (em termos da qualidade ou precisão de seus resultados); (ii) ser barato (em termos dos recursos utilizados para obter os resultados); (iii) ser rápido (em termos do tempo que leva obter os resultados). Segundo esse modelo, para um projeto só podemos escolher duas dessas características por vez. Assim, pode-se dizer que, ao otimizar duas dessas características, a outra vai ser afetada (piorada). Desse modo, para se obter resultados relevantes, uma das três características terá que ser relaxada.

Como foi indicado, existem diversas técnicas para fazer análise de desempenho (em função das caraterísticas do sistema a ser modelado), assim podemos encontrar técnicas com bons resultados e que são rápidas, mas muito custosas. Outras técnicas possuem bons resultados e são baratas, mas não rápidas. E existem também aquelas que são rápidas e baratas, mas não geram resultados tão bons. Em qualquer um desses casos, a técnica de análise não deixa de atender a um requisito, ela apenas não o atende de maneira ótima.

Já que na modelagem analítica temos as técnicas baseadas em processos de Markov, as quais modelam um sistema e examinam cada um dos seus possíveis estados para medir o desempenho, podemos qualificar essa técnica como boa (apesar de ser um abstração do modelo real) e barata (desenvolvimento do modelo), porém não é rápida (computar os resultados) por causa do problema de explosão do espaço de estados. Por conseguinte, no contexto da análise de desempenho preditiva, se desejamos construir um modelo analítico capaz de lidar com o problema da explosão do espaço de estados (ou seja, que seja viável computacionalmente), temos como requisito candidato a ser relaxado a precisão dos resultados obtidos na análise.

\subsection{Objetivos}

Neste trabalho, estamos interessados em predizer o desempenho de sistemas de workflows em que temos uma grande quantidade de instâncias de um mesmo workflow sendo executadas concorrentemente, disputando o acesso a um conjunto limitado de recursos computacionais de uso exclusivo do workflow.

Nosso objetivo é propor um novo método para a análise preditiva desse tipo de sistema. A intenção desse método é gerar modelos analíticos que:

- evitem o problema do rápido crescimento do espaço de estados;

- representem as principais caraterísticas de um sistema de workflow e de seus recursos;

- forneçam estimativas de desempenho confiáveis, o mais aproximadas das soluções existentes;

- possam ter sua solução computada em um tempo viável.

Para isso, desenvolvemos modelos baseados na Teoria do Campo Médio (TCM). A Teoria do Campo Médio [OW00, OS01] é uma técnica de análise que visa aproximar o comportamento de um sistema complexo de vários estados, composto por muitas entidades similares que interagem entre si, sem necessidade de representar explicitamente cada interação das entidades. Para isso, procura-se substituir todas as interações entre as entidades por uma interação média ou efetiva (campo médio). 
O modelo Markoviano de um workflow de numerosas instâncias concorrentes tem um espaço de estados discreto e de ordem exponencial, o que torna a sua análise um problema computacionalmente intratável. Por meio de técnicas baseadas na Teoria do Campo Médio, pode-se criar um sistema determinístico (aproximação determinística), com espaço de estados contínuo, cujo comportamento aproxima o do modelo estocástico, quando o modelo estocástico respeita certas condições. Dessa forma, evita-se o problema da explosão do espaço de estados, sendo possível calcular medidas de desempenho em tempos viáveis.

O método indica que a solução estocástica e a determinística são iguais quando se tem infinitas entidades. Porém, mesmo para quantidades finitas de entidades o modelo determinístico é uma boa aproximação.

Neste trabalho, temos ainda os seguintes objetivos mais específicos:

- A partir de um modelo de workflow, gerar um modelo Markoviano, expressando suas especificações de recursos;

- Determinar a aproximação determinística do modelo Markoviano do workflow, verificando as condições necessárias para isso, bem como identificar as consequências (na modelagem e na análise do sistema) das possíveis restrições impostas pela aproximação;

- Desenvolver um método para representar o workflow como um sistema determinístico;

- Determinar como serão extraídos (calculados) os índices de desempenho a partir da aproximação determinística.

\subsection{Contribuições}

As principais contribuições deste trabalho são:

- Demonstração teórica e experimental do problema da explosão de estados presentes em modelos Markovianos para workflows com numerosas instâncias e quantidade de recursos limitada.

- Representação de um workflow por meio de um sistema determinístico, que é a aproximação do modelo estocástico do workflow. Foram verificadas as condições sob as quais existe a aproximação determinística para o sistema estocástico.

- Método de mapeamento de um modelo de workflow descrito em Business Process Model and Notation (BPMN) para um sistema determinístico, descrito por um conjunto de Equações Diferenciais Ordinárias (ODEs).

- Extração de métricas de desempenho para workflows com numerosas instâncias e quantidade de recursos limitados, por meio de um sistemas determinístico, realizada em tempos computacionais viáveis.

\subsection{Organização do Texto}

No Capítulo 2, apresentamos os conceitos básicos relacionados aos workflows, descrevemos as perspetivas sob as quais são implementados e a sua representação com a notação BPMN. Também introduzimos conceitos de análise de sistemas computacionais, com destaque na análise preditiva 
de desempenho. Depois, dando enfase à modelagem analítica, descrevemos os processos estocásticos e as cadeias de Markov, assim como as principais ferramentas baseadas em sistemas estocásticos.

A modelagem analítica de workflows usando modelos Markovianos é discutida no Capítulo 3, que também trata da análise de desempenho e descreve formalmente o problema da explosão do espaço de estados, presente nessa abordagem.

O Capítulo 4 introduz conceitos da Teoria do Campo Médio e de sistemas determinísticos. Nesse capítulo, especificamos as condições para se ter a aproximação de sistemas estocásticos. Em seguida, apresentamos o método proposto neste trabalho para representar um workflow por meio de um sistema determinístico.

A análise de desempenho em sistemas determinísticos é explicada no Capítulo 5, onde são apresentados modelos de workflow e suas respectivas predições de desempenho.

Por fim, no Capítulo 6 listamos as conclusões sobre os resultados alcançados. 


\section{Capítulo 2}

\section{Conceitos}

O gerenciamento de workflows envolve diversos desafios, desde a representação do sistema até a sua execução, em que pode-se utilizar diferentes estratégias para se tomar uma decisão. Para realizar uma atividade, por exemplo, pode-se necessitar de diferentes recursos em diversas quantidades. Dessa forma, deve-se considerar a disponibilidade deles e a quantidade de trabalho que terão que processar.

Nos experimentos deste trabalho, busca-se realizar a predição de desempenho de um sistema composto por múltiplas instâncias de um workflow compartilhando um conjunto de recursos limitado, de modo a se ter conhecimento - com base nos indicadores de desempenho - do número de recursos necessários para a sua execução. Espera-se que, com os recursos provisionados, seja possível executar as atividades em tempos razoáveis, sem que recursos sejam sobrecarregados ou estejam ociosos.

Assim, neste capítulo são apresentados os conceitos fundamentais relacionados à análise de desempenho preditiva de workflows, contexto no qual a pesquisa foi realizada; modelagem de workflows, recursos e requisitos para sua execução; análise de sistemas computacionais; seleção de métricas de desempenho que avaliam o comportamento do workflow; introdução aos processos estocásticos, principal ferramenta de análise de desempenho; e Teoria do Campo Médio, que lida com o problema da explosão de estados na análise de desempenho preditiva de workflows.

\subsection{Workflow}

Um workflow é a automação total ou parcial de um conjunto de atividades que são executadas sob alguma especificação de controle de fluxo, para atingir um objetivo específico [Hol95]. É comum ver na literatura da área esse termo ser usado como um sinônimo para processo de negócio, pois o conceito de workflow nasceu na década de 90 em um contexto industrial, como uma solução para aliviar o problema de organização na execução de tarefas administrativas e de produção [VDAVH04, BC14, vdAtHKB03]. Entretanto, nos últimos anos, os workflows também vêm sendo bastante empregados em domínios acadêmicos na automação de experimentos científicos.

Segundo a Workflow Management Coalition (WfMC), entidade fundada em 1993 para padronizar e desenvolver os conceitos relacionados à sua modelagem, workflow pode ser definido como $a$ automação de um processo de negócio, total ou parcial, na qual documentos, informações ou tarefas são passadas entre participantes de acordo com um conjunto de regras procedurais para atingir ou contribuir em um objetivo do negócio [Hol95].

Um Sistema Gerenciador de Workflows (WFMS, do inglês Workflow Management System) é um software que apoia a criação, o gerenciamento e a execução de workflows. Um WFMS controla a ordem de execução das atividades de acordo com a representação computacional da definição 
do workflow, além de intermediar a interação com os participantes de workflow e com outros sistemas se for preciso [WfM99]. Um WFMS também atua como uma ferramenta administrativa e de supervisão do trabalho realizado nos workflows.

\subsubsection{Fluxo de Controle $\times$ Fluxo de Dados}

Sendo os workflows constituídos por conjuntos de atividades, tais atividades têm que interagir entre si. As formas de se modelar e implementar essas interações, em geral, podem ser classificadas sob duas perspectivas: fluxo de controle e fluxo de dados. No fluxo de controle, a interação das atividades representa a transferência do controle de uma (ou mais) atividade(s) à(s) atividade(s) subsequente(s). Assim, as estruturas de controle podem ser vistas como sequências, iterações e desvios condicionais. Já o fluxo de dados representa as transferências de dados entre as atividades, de forma que as atividades consumidoras (aquelas que recebem os dados em um determinado momento) serão dependentes das atividades produtoras (as que produzem os dados).

Existem outras perspectivas além dessas duas. Uma delas é a organizacional (também chamada de recursos), onde se tem uma visão da estrutura do workflow de acordo com os recursos que irão executar as atividades. Também há a perspectiva operacional, que descreve as ações elementares, e especifica e coordena ferramentas e aplicações que realizarão as ações [RS07]. A perspectiva de tratamento de exceções visa lidar com as causas das exceções e definir as ações de correção a serem tomadas em caso de falhas [vdABtHK00, vdAtHKB03].

Um processo de negócio é modelado geralmente sob a perspectiva de fluxo de controle. Porém, essa perspectiva não é totalmente independente das outras, já que elas são complementares. O fluxo de controle é a principal forma de termos uma visão clara dos objetivos do processo e podermos avaliar sua efetividade. No entanto, as outras perspectivas auxiliam na especificação do processo.

Serão de maior interesse neste trabalho workflows modelados sob uma perspetiva de fluxo de controle, pois ela permite um maior foco na execução das atividades e ajuda na compreensão do desempenho do sistema.

\subsubsection{Modelagem de Workflows}

Os processos de negócio têm muitas características comuns, mas muitas vezes eles têm propriedades próprias e específicas no contexto no qual se aplicam. Nesse sentido, abordar todas as características relevantes em um modelo abstrato pode ser uma tarefa complicada, pois tal estrutura deve ser capaz de representar a informação o mais perto possível da realidade. Como definido na Seção 2.1, um workflow é uma automação de um processo de negócio, baseada em sua representação abstrata. A seguir, são definidos termos básicos relacionados a essa representação [BC14, RvdATHE05]:

- Modelo de workflow - define quais são e como serão executadas as atividades para realizar um determinado objetivo. O modelo do workflow geralmente define a ordem, ainda que parcial, em que as atividades devem ser executadas. Para representá-lo são usadas comumente duas abordagens: (i) uma linguagem de especificação de workflows, que permite a especificação de processos com um nível de detalhamento próximo ao requerido para sua execução; (ii) um arcabouço de modelagem formal, que possibilita a análise de propriedades dos processos modelados. 
- Atividade - é a unidade mínima de trabalho, isto é, que não precisa ser decomposta em outras atividades menores dentro de um processo. Existem três tipos de atividades: manual, semi-automática e automática. As atividades manuais dependem de recursos humanos para serem realizadas. As automáticas dependem unicamente de recursos computacionais. Já as semi-automáticas dependem dos dois tipos de recursos. As atividades podem também ser classificadas como: atômica, de bloco, de múltiplas instâncias e de bloco com múltiplas instâncias.

- Conector - é usado para interligar as atividades no modelo de workflow, definindo o seu fluxo de execução. Uma ligação entre atividades indica que uma depende da outra. Em outras palavras, a execução de uma atividade pode estar condicionada à execução prévia de outras atividades no modelo. As linguagens de modelagem de workflows podem ter o seu próprio conjunto de conectores, que denotam a expressividade da linguagem para representar os diversos modelos de workflows.

- Instância de workflow - é a realização de uma simples execução do workflow, incluindo os dados associados a essa execução. Cada instância é criada e controlada independentemente pelo sistema gerenciador de workflows. Também pode ser chamada de caso, porque se refere ao estado de uma execução particular do processo.

- Instância de atividade - representação de uma execução particular de uma atividade dentro de uma instância de workflow. Assim, uma instância de atividade é criada na invocação de uma atividade dentro da execução de uma instância de workflow e usa os dados associados à instância à qual pertence.

Na execução das atividades de um workflow sob o fluxo de controle, podemos ter 4 tipos básicos de comportamento, chamados também de roteamento [VDAVH04]:

- A forma mais simples é a execução sequencial de atividades, ou seja, elas são executadas uma após a outra. Dessa forma, torna-se mais clara a dependência entre as atividades, pois a execução de certas atividades dependem de outras que já foram executadas na sequência.

- A execução paralela refere-se à execução de atividades de forma simultânea. Por serem ao mesmo tempo, tais tarefas terão de ser independentes, com o resultado de uma não afetando a execução das outras.

- Há também a execução seletiva ou condicional, quando é necessário escolher entre duas ou mais atividades. A escolha dependerá de condições preestabelecidas sobre a instância do workflow.

- Temos por último a execução iterativa, comportamento que se procura evitar. Representa a execução de uma atividade ou de um bloco de atividades mais de uma vez.

A maneira de se representar um workflow depende muito do processo a ser modelado, assim como dos requisitos a serem cumpridos. Logo, o modelo do workflow, composto por atividades conectadas na forma de um grafo dirigido, deve representar e detalhar da melhor forma possível as informações de interesse. 
Para este trabalho, a interação de atividades será descrita por meio do fluxo de controle. Dessa forma, o modelo de workflow será descrito na linguagem Business Process Model and Notation (BPMN).

\subsection{Business Process Model and Notation (BPMN)}

Business Process Model and Notation (BPMN) [OMG11] é um padrão desenvolvido pelo Object Management Group (OMG) [OMG]. Prevê uma notação facilmente compreensível por todos os usuários do negócio, desde os analistas que criam os esboços do processo, e os técnicos que desenvolvem o ambiente para a sua execução, até as pessoas que irão gerenciá-lo e monitorá-lo [OMG11]. Para atingir essa especificação, foi necessário o estudo de metodologias já maduras na área de modelagem de processos de negócio (como Unified Modeling Language (UML), ActivityDecision Flow (ADF) Diagram, RosettaNet, LOVeM e Event-Process Chains (EPC)), colhendo assim as melhores práticas delas numa única notação padrão.

Os diagramas BPMN podem ser de três tipos: diagramas de processos também chamados de diagramas de fluxo de controle, representa o fluxo de execução de um processo; diagramas de colaboração, descreve a interação de dois ou mais processos de negócio; e diagramas de coreografia, onde uma coreografia é a definição do comportamento esperado de participantes que interagem entre si. Um processo é definido em BPMN como uma sequência ou fluxo de atividades em uma organização que tem como objetivo executar um trabalho. Essa definição é similar à de workflow, apresentada na Seção 2.1, portanto neste texto os termos workflows e processos de negócio serão usados indistintamente. Os diagramas de processos são de maior utilidade neste trabalho, já que estamos interessados em modelos de workflows para análise de processos de negócio com numerosas instâncias e recursos compartilhados.

Um processo é modelado em BPMN como um grafo de elementos de fluxo que estão categorizados em atividades, eventos, desvios e fluxos de sequência e que definem semânticas de execução finitas. Pelo fato de BPMN ser uma notação robusta, cada uma dessas categorias de elementos de fluxo podem ter vários tipos de componentes ao mesmo tempo, para uma descrição detalhada dos processos. Contudo, neste trabalho nos restringiremos a um subconjunto desses componentes. Os elementos escolhidos permitem modelar workflows compostos pelos quatro tipos de comportamentos básicos especificados para fluxo de controle. Na continuação, são descritos tais componentes em BPMN.

\subsubsection{Fluxo de sequência}

O fluxo de sequência indica a transferência do controle entre elementos do processo, ou seja, determina o fluxo de execução das suas atividades. Por meio dele, podemos modelar o primeiro comportamento, ou roteamento, definido no fluxo de controle de workflows, i.e., a execução sequencial.

\subsubsection{Tarefas}

Uma tarefa é uma atividade atômica dentro do processo, i.e., ela representa um trabalho que não pode ser dividido em níveis mais refinados de detalhamento. Geralmente, são os recursos que executam as tarefas. 


\subsubsection{Eventos}

Um evento é algo que "acontece" durante a execução do processo, afetando seu fluxo, e geralmente tem uma causa e um efeito, ou seja, requer uma ação e produz outra. Existem três tipos principais de eventos: de início, de fim e intermediários. Todavia, utilizaremos apenas os dois primeiros. O evento de início indica onde o processo começa, ou seja, determina o começo do seu fluxo, logo, não tem nenhum fluxo de sequência como entrada. Por outro lado, o evento de fim, como o nome indica, representa o fim do processo, ou seja, determina o fim de seu fluxo, portanto, não tem fluxo de sequência como saída.

\subsubsection{Desvios}

Os desvios são usados para controlar como os fluxos de sequência interagem, convergindo ou divergindo dentro do processo, pudendo então o desvio exercer ambas funções, ou seja que tenha múltiplos fluxos de entrada e saída. Uma boa prática de modelagem declara que um desvio só deve desempenhar uma dessas funções. Neste trabalho é muito importante cuidar para que um desvio exerça apenas uma das funções: convergência (ter múltiplas entradas) ou divergência (ter múltiplas saídas). Existem diferentes tipos de desvios, mas neste trabalho utilizaremos dois deles:

- Desvio exclusivo - O desvio exclusivo de divergência serve para criar caminhos alternativos no fluxo do processo. A instância do processo só poderá continuar em um desses caminhos. Para determinar o caminho, em primeiro lugar, deve ser feita uma pergunta em algum ponto da instância do processo, associando cada possível resposta a um fluxo de saída do desvio exclusivo. Por outro lado, o desvio exclusivo de convergência conecta caminhos alternativos.

O desvio exclusivo permite modelar outros dois tipos de comportamento do fluxo de controle do workflow: a execução seletiva e a execução iterativa. No modelo de workflow, os desvios exclusivos de divergência e convergência são chamados também de XOR-split e XOR-join, respetivamente.

- Desvio paralelo - O desvio paralelo de convergência é usado para sincronizar (combinar) fluxos paralelos, já o desvio paralelo de divergência cria os fluxos paralelos.

A execução paralela no fluxo de controle do workflow pode ser modelada por meio do desvio paralelo. No modelo de workflow, o desvio paralelo de divergência é conhecido como ANDsplit, e o de convergência, $A N D$-join.

As representações gráficas dos elementos descritos anteriormente são mostradas na Tabela 2.1. Os desvios de convergência podem ter mais de um fluxo de sequência como entrada, assim como os de divergência como saída. Nas figuras, foram colocados apenas três como exemplo em ambos os casos.

Como mencionado antes, pelo fato do processo em BPMN ser um grafo de elementos de fluxo, neste trabalho consideramos que o modelo de um workflow é um grafo dirigido. Os vértices do grafo podem ser de três tipos: eventos, tarefas e desvios, os quais foram descritos anteriormente. Já uma aresta do grafo representa um fluxo de sequência. A fim de evitar possíveis ambiguidades semânticas, pelas combinações que a união desses elementos podem gerar, o modelo de workflow deve respeitar as seguintes regras: 


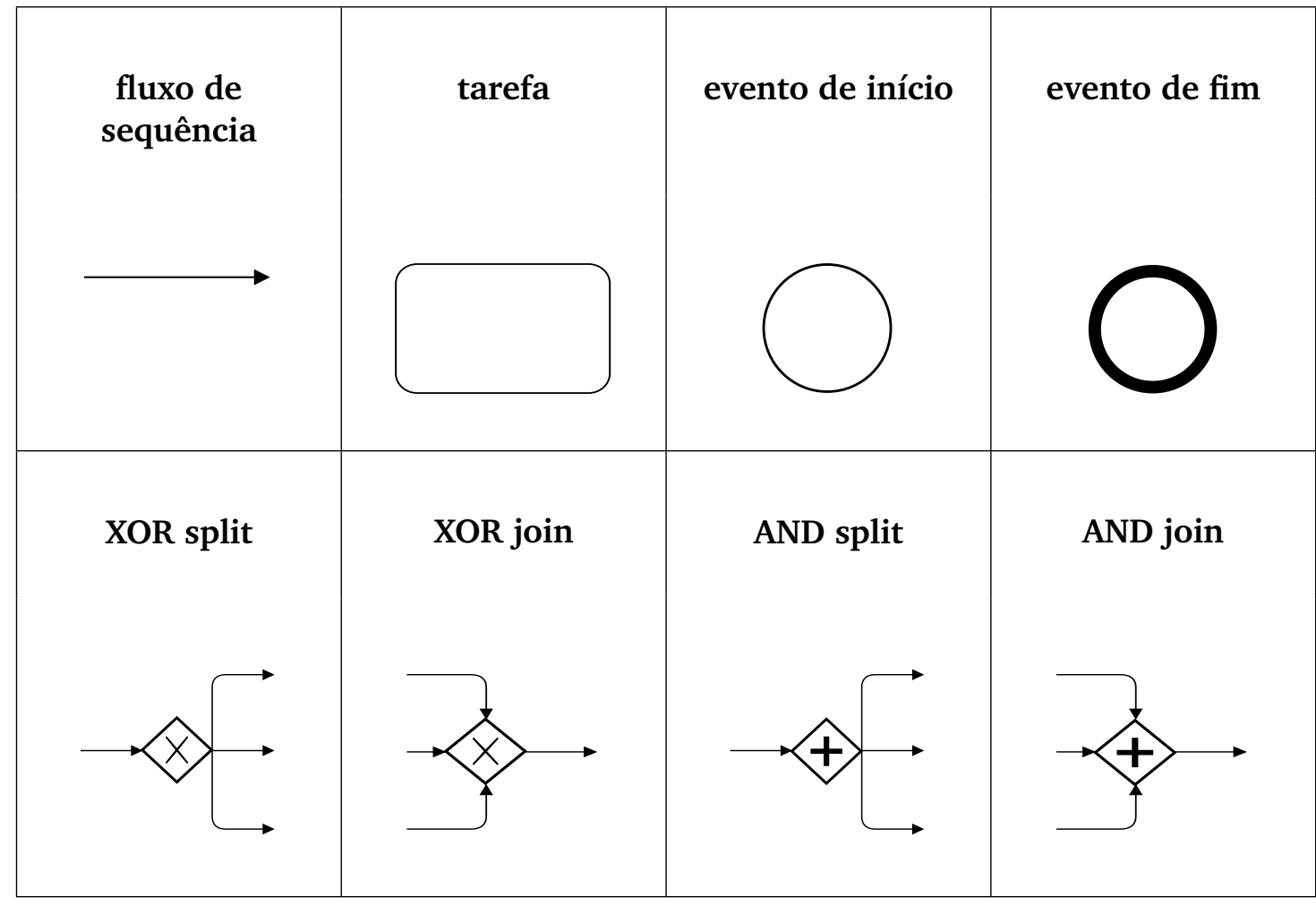

Tabela 2.1: Elemento de fluxo do BPMN usados neste trabalho

- apenas um vértice pode ser do tipo evento de início, tendo uma só aresta de saída e nenhuma de entrada;

- apenas um vértice pode ser do tipo evento de fim, tendo uma só aresta de entrada e nenhuma de saída;

- todo vértice do tipo tarefa deve ter uma aresta de entrada e outra de saída;

- todo vértice do tipo desvio de divergência deve ter uma aresta de entrada e duas ou mais de saída;

- todo vértice do tipo desvio de convergência deve ter apenas uma aresta de entrada e mais de uma de saída;

- todo vértice XOR-join não pode unir caminhos paralelos;

- da mesma forma, todo vértice $A N D$-join não pode sincronizar caminhos alternativos;

- para todo vértice $v$ que não é um evento: (i) deve existir pelo menos um caminho do vértice evento de início para $v$, e (ii) deve existir pelo menos um caminho de $v$ ao vértice evento de fim;

- toda sequência de fluxo de saída de um vértice XOR-split deve ter associada a ela uma probabilidade de execução;

- a soma das probabilidades de todas as saídas de um vértice XOR-split deve ser 1. 
Um modelo de workflow que cumpre todas essas restrições é denominado bem-definido, de acordo com a Workflow Patterns Initiative ${ }^{1}$. É importante destacar que esse tipo de modelo não permite a existência de vértices do tipo tarefa que nunca serão executados. Ele também garante que toda instância de workflow possa terminar. Essas características são determinadas especialmente pela existência de caminhos entre o evento de início e os demais vértices, e entre esses vértices e o evento de fim. Portanto, pode-se concluir que para todo vértice $v$, existe pelo menos um caminho que acaba (que tem como origem o evento de início e como último vértice o evento de fim) e que contém $v$. Um exemplo simples de modelo de workflow bem-definido é apresentado no diagrama da Figura 2.1.

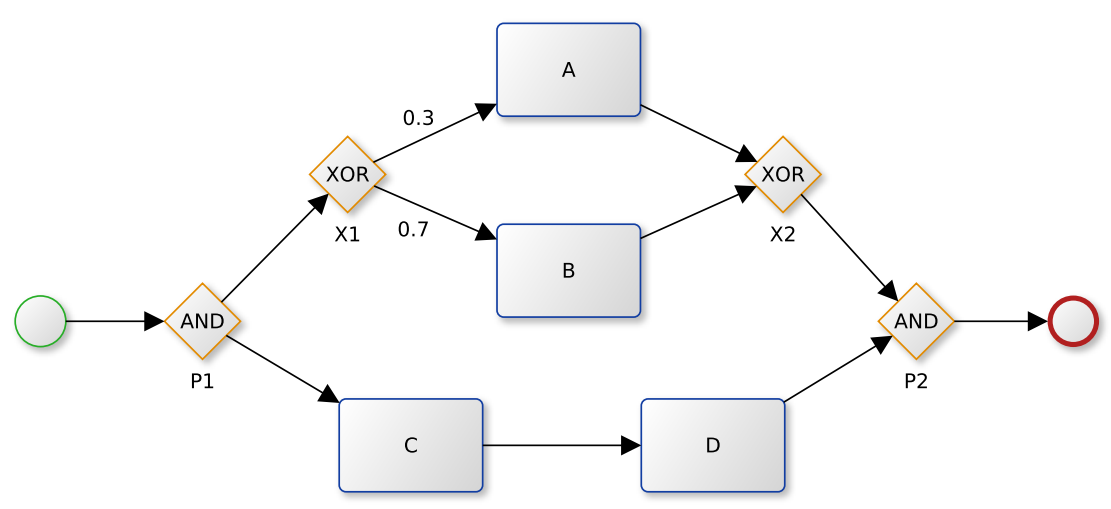

Figura 2.1: Modelo de workflow bem-definido em notação BPMN.

Dessa forma, por meio da representação do workflow com a notação BPMN, temos uma ideia clara da estrutura do processo sob a perspetiva de fluxo de controle. No entanto, até agora o fluxo de execução só leva em conta o trabalho a ser realizado, faltando um fator importante na descrição do processo, a saber, os recursos que irão executar o trabalho. Esse assunto será abordado na próxima seção.

\subsection{Recursos no Workflow}

Nos workflows, sob uma perspetiva de controle de fluxo, o termo trabalho possui uma interpretação intuitiva. O mesmo não ocorre para o termo recurso, cuja definição não é tão clara assim. Dado que um trabalho tem que ser feito por algo ou alguém, então, no contexto de workflow, podese considerar que um recurso é uma entidade que é capaz de realizar uma atividade, ou seja, de trabalhar. Um dos maiores objetivos na atualidade é desenvolver o trabalho de forma que os recursos envolvidos sejam usados da maneira mais otimizada possível, utilizando a menor quantidade possível de recursos que possam atingir sua máxima capacidade e ao mesmo tempo não deixar que o trabalho demore muito para ser executado.

Em workflows, podemos identificar dois tipos de recursos: recursos humanos e não humanos. Os recursos humanos normalmente desenvolvem tarefas que precisam de maior atenção na sua execução e cuja automatização não é factível. As tarefas que podem ser automatizadas são executadas por recursos não humanos (e.g., máquinas ou sistemas computacionais). Além de serem classificados como humanos ou não humanos, os recursos podem ser categorizados quanto aos tipos de tarefas que podem executar, ou ao nível em que desenvolvem as tarefas. Também pode-se classificá-los em grupos nos quais cada um tem diferentes papéis, ou ainda classificar os recucursos pelo modo de se comunicarem. [RHE04].

\footnotetext{
${ }^{1}$ Workflow Patterns Initiative: http://www.workflowpatterns.com/
} 
No presente trabalho, são de maior relevância os recursos computacionais, pois o objetivo é a execução de workflows em um ambiente com esse tipo de recurso em quantidade e com capacidade limitadas. Uma das principais características dos recursos computacionais é o fato de terem um limite na capacidade de executar tarefas, ou seja, os recursos nem sempre são capazes de executar um trabalho no mesmo instante em que são requisitados, seja pelo fato de já estarem executando uma outra tarefa (no caso de recursos que só podem executar uma tarefa por vez), ou pela capacidade máxima deles para processar trabalhos já ter sido atingida.

Um dos desafios na modelagem de workflows é a representação dos recursos. A inclusão da descrição dos recursos no modelo é fundamental para a análise preditiva do desempenho de workflow. Os requisitos de recursos das atividades que formam o workflow estão envolvidos com o tempo de execução das instâncias do workflow. Além disso, a carga de trabalho no sistema afeta diretamente o tempo de execução. À medida que aumentamos a quantidade de trabalho, mais recursos são usados, fazendo com que a capacidade máxima do sistema esteja cada vez mais próxima de ser atingida e causando até a interrupção do funcionamento do sistema em situações de sobrecarga.

Neste trabalho, a representação de recurso que usaremos será a proposta em [Bra11], definida a seguir:

Definição 2.3.1. Um recur so é representado como uma tupla ([id recurso], [quantidade], [capacidade de trabalho]), onde:

- [id recurso] é o identificador que especifica o tipo de recurso;

- [quantidade] indica o número disponível (unidades) do recurso;

- [capacidade de trabalho] representa, em média, a quantidade de trabalho que uma unidade do recurso pode executar por unidade de tempo.

Já que a [capacidade de trabalho] é uma das especificações do recurso, é importante descrever a qual trabalho estamos nos referindo, ou seja, com qual atividade, ou quais atividades, está relacionada essa capacidade de trabalho. Assim, para cada atividade do workflow é necessário detalhar os recursos de que precisa para ser executada. Dessa forma, definimos os requisitos das atividades.

Para representarmos os requisitos são necessárias as seguintes definições:

Definição 2.3.2. Uma fórmula, ou fórmula bem formada do cálculo proposicional, também chamada de fórmula proposicional, é uma sequência finita de símbolos de um determinado alfabeto [Men09]. O alfabeto é composto de três tipos de símbolos:

- As variáveis proposicionais. Neste trabalho, uma variável proposicional é definida como sendo a tupla ([id recurso], [quantidade]) que representa a quantidade de trabalho de alguma atividade a ser executada pelo recurso representado por [id recurso]. Nesse caso, a variável proposicional será verdadeira se existir pelo menos uma unidade do recurso mencionado. Caso contrário, será falsa.

- Os conectivos proposicionais. Neste contexto, serão necessários só dois: a conjunção e a disjunção representados, respectivaemnte, por $\{\wedge, \vee\}$.

- Os parênteses \{“(”, “)”\}.

Assim, a fórmula está definida da seguinte maneira: 
- Cada variável proposicional é uma fórmula, por si só.

- Se $\alpha$ e $\beta$ são fórmulas, $e \otimes$ é um conectivo, então $(\alpha \otimes \beta)$ também é uma fórmula.

Dada uma atividade A do workflow, o seu requisito é definido como sendo uma fórmula proposicional na forma normal disjuntiva (FND). Uma fórmula é considerada na FND se, e somente se, é a disjunção de uma ou mais conjunções, e cada conjunção é composta por uma ou mais variáveis proposicionais.

Exemplos de requisitos válidos são:

- $\left[R_{1}, 1\right]$. Nesse caso, a atividade precisa apenas do recurso $R_{1}$ para executar uma unidade de trabalho.

- $\left(\left[R_{1}, 1\right] \vee\left(\left[R_{2}, 2\right] \wedge\left[R_{3}, 3\right]\right)\right)$. Significa que a atividade precisa do recurso $R_{1}$ para realizar uma unidade de trabalho, ou de $R_{2}$ e $R_{3}$ para executar, respectivamente, 2 e 3 unidades de trabalho.

- $\left[R_{1}, 1\right] \vee\left[R_{2}, 2\right]$. A atividade só pode ser realizada com o recurso $R_{1}$ para uma unidade de trabalho, ou com o recurso $R_{2}$ para 2 unidades de trabalho.

- $\left(\left[R_{1}, 1\right] \wedge\left[R_{2}, 2\right]\right) \vee\left(\left[R_{1}, 1\right] \wedge\left[R_{3}, 3\right]\right)$. A atividade precisa de $R_{1}$ para realizar uma unidade de trabalho e $R_{2}$ para realizar 2 unidades de trabalho, ou $R_{1}$ para uma unidade de trabalho e $R_{3}$ para 3 unidades de trabalho.

Para uma atividade ser executada, os seus requisitos de recursos devem ser satisfeitos, isto é, a fórmula proposicional que define os requisitos deve ser verdadeira. Isso só se dará se pelo menos uma das conjunções que compõem a fórmula for verdadeira. Neste trabalho, assumimos que todos os recursos da conjunção verdadeira (as variáveis proposicionais), nos requisitos, serão alocados para a execução exclusiva da atividade que os solicitou. Da mesma forma, todos esses recursos que forem alocados serão liberados quando a execução da atividade for completada.

Tendo definido o sistema de workflow, os recursos que serão usados, assim como os requisitos de que cada uma das atividades que compõem o workflow precisa, é necessário avaliar o sistema como um todo. Dessa forma, na próxima seção veremos conceitos relacionados com análise de sistemas computacionais, os tipos de análise que podem ser feitos, os métodos para desenvolvê-las, quais parâmetros são necessários avaliar para a aplicação desses métodos, assim como a interpretação dos resultados produzidos.

\subsection{Análise de Sistemas Computacionais}

A análise de sistemas computacionais possibilita provar que os sistemas irão atender aos requisitos para os quais foram desenvolvidos. A análise de um sistema computacional não é uma tarefa simples e, dependendo do contexto, pode chegar a ser muito complexa. Alguns sistemas computacionais executam tarefas críticas. Nesses casos, para garantir que as tarefas sejam realizadas corretamente pelo sistema, é uma obrigação fazer a análise do sistema antes mesmo que ele comece a executar as tarefas.

A análise de sistemas computacionais não é usada apenas para comprovar que o sistema está correto semanticamente, ou seja, que ele cumpre o objetivo da tarefa que está executando, mas 
também para analisar as características sintáticas do sistema, isto é, aspectos relacionados ao tempo, ao uso dos recursos, à qualidade do serviço, entre outros.

Como já discutido no Capítulo 1, existem dois tipos principais de análise de sistemas computacionais:

- Análise Qualitativa - tipo de análise que está relacionada com a classificação dos aspectos funcionais do sistema. Normalmente, nesse tipo de análise, dá-se um nível de classificação a alguma característica que esteja sendo analisada.

- Análise Quantitativa - tipo de análise que visa medir as propriedades não-funcionais do sistema. Normalmente, nela são atribuídos valores numéricos às características que estão sendo analisadas.

Um dos principais tipos de análise quantitativa é a análise de desempenho [Jai91, NJ00, FM03], que pode ser feita por meio das seguintes técnicas:

- Medição - normalmente se dá quando já foi implementado o sistema que se quer analisar.

- Simulação - a partir do modelo do workflow, pode-se realizar simulações que imitem o processo de execução do workflow e, assim, avaliar as características do sistema.

- Modelagem analítica - nesse tipo de análise de desempenho, cria-se um modelo suficientemente formal do workflow, que depois é avaliado por meio de métodos matemáticos que permitem obter predições a respeito do desempenho do sistema.

É importante mencionar que cada uma delas requer diferentes características do ambiente em que está sendo aplicado. Assim, enquanto o uso de alguma técnica pode ser satisfatório numa determinada situação, pode ser inviável para outra. Então, escolher a aplicação de uma ou de outra técnica vai depender de vários fatores [Jai91, NJ00], entre os quais podemos destacar: o tempo disponível para a realização da análise, o nível de desenvolvimento em que se encontra o sistema, as ferramentas disponíveis, o nível desejado de exatidão das medições, o custo das técnicas, o nível de correlação das características e a popularidade (negociabilidade) da técnica.

O fator tempo é um dos mais importantes na hora de escolher a técnica de medida de desempenho. A análise por simulação é a mais demorada quando comparada com a modelagem analítica. Já no caso da análise por medição, a quantidade de tempo que se leva para aplicar a técnica é mais variada. O nível de desenvolvimento do sistema também condiciona o tipo de técnica a ser utilizada. A análise por medição exige que exista um sistema previamente desenvolvido. Já a modelagem analítica e a análise por simulação são mais flexíveis nesse sentido, pois podem ser usadas em qualquer sistema, independentemente do estado do sistema.

Um fator que influencia muito na escolha do tipo de técnica a se utilizar é a exatidão esperada das medições. Na modelagem analítica são feitas muitas suposições e simplificações que podem levar a resultados um pouco distantes dos reais. Já a análise por simulação precisa de menos simplificações para ser aplicada, e pode abranger mais detalhes na modelagem, o que faz com que os seus resultados sejam mais perto da realidade. A análise por medição é mais variável nesse sentido, pois depende muito de como foram feitos os experimentos no sistema já existente para obter as medições. Se os parâmetros do ambiente do sistema a ser avaliado forem parecidos aos 
dos experimentos realizados, a exatidão dos resultados será melhor, caso contrário os resultados obtidos podem não ser úteis. É importante destacar que as três técnicas podem produzir resultados errados ou de qualidade insuficiente para serem utilizados.

Dado que os sistemas de workflow modelam processos, para os quais é necessário medir o desempenho, podemos identificar as características deste tipo de sistema que determinam a escolha do tipo de técnica que será utilizado neste trabalho. Antes de mais de nada, precisamos de resultados que sejam obtidos em tempos razoáveis e que não precisem de um alto gasto de ferramentas para serem computados. Além disso, foram definidas antes os componentes do processo de uma forma formal, isto para facilitar a sua modelagem por meio de métodos formais, que por outro lado, essas definições formais involvem variáveis numéricas que servirão para determinar o desempenho do sistema. Tudo isso torna a modelagem analítica como a técnica a ser utilizada neste trabalho, pois por meio do uso de técnicas matemáticas visamos medir o desempenho dos workflows, isto requer o estudo de técnicas de modelagem abstratas e formais que serão detalhadas nas seções posteriores.

\subsection{Seleção de Métricas de Desempenho}

Segundo Jain [Jai91], uma forma de escolher as métricas de desempenho para um sistema é olhar as possíveis respostas que ele pode dar para uma requisição para realizar uma tarefa. Quando o sistema realiza a tarefa, há dois tipos de saída possíveis: (i) o sistema atendeu a requisição e gerou uma saída correta; e (ii) o sistema atendeu a requisição, mas gerou uma saída incorreta. Entretanto, o sistema também pode se negar a atender a requisição. Dessa forma, podemos ter os seguintes tipos de métricas de desempenho:

- Se o sistema realiza a tarefa e produz o resultado certo:

- Tempo de resposta - é o tempo que o sistema demora para responder corretamente a uma requisição do usuário.

- Rendimento - é o número de requisições atendidas em um intervalo de tempo.

- Utilização dos recursos - mede o uso de cada recurso em termos da porcentagem de tempo que é utilizada.

- Se o sistema realiza a tarefa, mas produz um resultado errado:

- Probabilidade do erro - serve para determinar a ocorrência de algum tipo de erro.

- Tempo entre erros - determina o intervalo de tempo entre a ocorrência dos erros.

- Se o sistema não atende à requisição:

- Duração do evento que não permite realizar o pedido - é o tempo que demora o evento que interrompe o uso do recurso para outras requisições.

- Intervalos entre os eventos - é o intervalo de tempo em que o sistema não atende a uma requisição, ou seja, o tempo entre as duas requisições que foram atendidas.

Por outro lado, cada uma dessas métricas de desempenho, de acordo com a função de utilidade, pode ser categorizada de três formas: 
- Mais alto é melhor - quanto mais alto for o valor da métrica, melhor será o resultado, e.g., o rendimento do sistema.

- Mais baixo é melhor - de preferência é melhor ter valores baixos para as métricas, e.g., o tempo de resposta.

- Nominal é o melhor - valores médios são preferidos, e.g., a utilização de recursos.

Para nosso trabalho de pesquisa são de maior interesse aqueles sistemas com a capacidade de responder às requisições produzindo o resultado esperado. Consequentemente, são de maior relevância as métricas de tempo de resposta, rendimento e a utilização de recursos. Como mencionado anteriormente, elas variam na sua função de utilidade, requirindo, portanto, uma análise individual e detalhada para cada uma delas.

Já que a análise de desempenho encontra-se dentro da análise quantitativa de sistemas computacionais, nosso objetivo é realizá-las nos workflows, com foco na técnica de modelagem analítica. Nas seguintes seções, veremos uma das principais técnicas para isso, as Cadeias de Markov, assim como as principais ferramentas desenvolvidas para modelar os sistemas e analisá-los.

\subsection{Processos Estocásticos}

Uma das técnicas mais utilizadas na modelagem analítica para a avaliação de desempenho de sistemas computacionais são os processos estocásticos, que têm um alto nível de formalismo e permitem o desenvolvimento de abordagens matemáticas para analisá-los.

Os sistemas do mundo real evoluem no tempo, mudando de estado em intervalos de tempo ou continuamente. É possível identificar dois tipos clássicos de sistemas. Os primeiros são os sistemas determinísticos, aqueles em que a evolução do sistema é determinada por uma regra fixa bem definida, tornando-se possível saber os estados que o sistema atingirá a partir do estado inicial e da regra de evolução. No segundo tipo, estão os sistemas estocásticos, também chamados de aleatórios, cuja evolução apresenta indeterminismo, isto é, mesmo conhecendo o estado inicial do sistema, é impossível ter certeza do próximo estado em que o sistema chegaria, pois existem diferentes direções para as quais o sistema pode evoluir.

Os sistemas de workflows são não-determinísticos, pois não é possível saber com exatidão quais serão suas entradas (as requisições dos usuários), e também não é possível conhecer as próximas saídas, ou seja, determinar os próximos estados do sistema. Dessa forma, torna-se factível o estudo do comportamento de workflows por meio de processos estocásticos.

Seja $(\Omega, F, \mathbb{P})$ um espaço de probabilidade, onde:

- $\Omega$ representa o espaço amostral (conjunto de todas as possíveis saídas para um experimento aleatório);

- F é um conjunto de eventos no experimento, onde os elementos de saída de um evento pertencem a $\Omega$;

- $\mathbb{P}$ denota uma função que retorna a probabilidade de um evento acontecer. Ela fornece valores entre 0 e 1 , isto é, $\mathbb{P}: F \rightarrow[0,1]$.

Logo, podemos definir um processo estocástico da seguinte forma [BW90, $\mathrm{R}^{+}$96]: 
Definição 2.6.1. Seja $T$ um subconjunto de $[0, \infty)$. Um processo estocástico indexado por T é definido como uma coleção de variáveis aleatórias $\left\{X_{t}\right\}_{t \in T}$, em um espaço de probabilidade $(\Omega, F, \mathbb{P})$, que assumem valores em um conjunto $S$, onde $S$ é o espaço de estados do processo.

É importante distinguir entre os dois tipos de processos estocásticos possíveis devido à natureza do conjunto $T$. Quando $T=\mathbb{N}$, então diz-se que o processo estocástico é de tempo discreto, e quando $T=[0, \infty]$ ou $T=[a, b] \subset \mathbb{R}$, o processo é de tempo contínuo.

Quando $T$ é um conjunto unitário, $T=\{1\}$, o processo $\left\{X_{t}\right\}_{t \in T} \equiv X_{1}$ é uma simples variável aleatória. Logo, quando $T$ é um conjunto finito, $T=\{1,2, \ldots, n\}$, o processo pode ser visto como sendo um vetor aleatório, porém ele é uma generalização dos vetores aleatórios. A principal diferença a destacar é que as variáveis envolvidas no vetor podem representar diferentes coordenadas espacias. No entanto, no processo estocástico leva-se em consideração o tempo, ou seja, a interpretação que é dada para $t \in T$ é diferente para os vetores aleatórios, se comparada com a dos processos estocásticos.

\subsubsection{Cadeias de Markov}

Muitos sistemas do mundo real têm sido estudados e modelados sob a perspectiva dos processos estocásticos. Todavia, um grupo de sistemas apresenta uma propriedade interessante que ajuda na sua modelagem. A propriedade em questão se refere ao fato de que, muitas vezes, para se conhecer os próximos estados do sistema (estados futuros para os quais o sistema pode evoluir), não seria necessário conhecer todos os anteriores, já que a partir do estado atual seria possível avaliar os futuros.

\subsubsection{Processo de Markov}

Dado um processo estocástico $\left\{X_{t}\right\}_{t \in T}$, e seja $n$ um dado tempo $(n \in T)$ tal que $X_{n}$ denota o estado atual ou "presente" do processo, pode-se definir $\left(X_{t} ; t<n\right)$ como o "passado", e $\left(X_{t}, t>\right.$ $n)$ o "futuro" do processo. Para saber o que vai acontecer no futuro do processo, geralmente é importante saber o seu passado e presente. Mas no caso de uma sequência de variáveis aleatórias independentes, ou de um simples passeio aleatório, por exemplo, tal condição não chega a ser necessária, já que se pode considerar o passado como irrelevante para o cálculo do comportamento futuro do processo. Portanto, nesses casos, o estado futuro depende apenas do estado mais recente do processo [BW90, Kar14, Dur12]. Apresentamos a definição de processo de Markov a seguir:

Definição 2.6.2. Um processo estocástico $\left\{X_{t}\right\}_{t \in T}$ com espaço de estados $S$ é um processo de Markov se respeita a propriedade de Markov. A propriedade de Markov requer que todo o passado possa ser concentrado no último evento de modo a considerá-lo no evento seguinte. Isto é, para qualquer $t_{q} \geqslant$ $t_{p} \geqslant t_{n-1} \geqslant \ldots \geqslant t_{0}$ com $t_{q}, t_{p}, t_{i} \in T(0 \leqslant i \leqslant n-1) e\left\{j, i, i_{n-1}, \ldots, i_{0}\right\} \in S$, vale:

$$
\mathbb{P}\left(X_{t_{q}}=j \mid X_{t_{p}}=i, X_{t_{n-1}}=i_{n-1}, \ldots, X_{t_{0}}=i_{0}\right)=\mathbb{P}\left(X_{t_{q}}=j \mid X_{t_{p}}=i\right)
$$

Para o caso particular de um processo estocástico de tempo discreto, temos que:

$$
\mathbb{P}\left(X_{t_{n+1}}=j \mid X_{t_{n}}=i, X_{t_{n-1}}=i_{n-1}, \ldots, X_{t_{0}}=i_{0}\right)=\mathbb{P}\left(X_{t_{n+1}}=j \mid X_{t_{n}}=i\right)
$$

Portanto, existem dois tipos de processos de Markov: de tempo discreto e de tempo contínuo.

Uma Cadeia de Markov é definida como um processo de Markov onde o espaço de estados $S$ é 
contável. Logo, em função do tipo de processo de Markov podem existir dois tipos de Cadeias de Markov:

- Cadeia de Markov de Tempo Discreto - CMTD (Discrete Time Markov Chain ) - se o processo de Markov é de tempo discreto.

- Cadeia de Markov de Tempo Contínuo - CMTC (Continuous Time Markov Chain) - se o processo de Markov é de tempo contínuo.

A propriedade de Markov pode contribuir para um menor uso de memória em cada estado. Isso se torna mais claro nas CMTCs, dada a propriedade da homogeneidade de tempo [Ros14], que é definida da seguinte forma: sejam $t_{p}, t_{q} \in T$ tal que $t_{p} \leqslant t_{q}$ e quaisquer $i, j \in S$,

$$
\mathbb{P}\left(X_{t_{q}}=j \mid X_{t_{p}}=i\right)=\mathbb{P}\left(X_{t_{q}-t_{p}}=j \mid X_{0}=i\right) .
$$

Uma CMTC na qual o número de transições é finito com probabilidade 1, em qualquer intervalo de tempo finito, é chamada de CMTC regular.

Seja $p_{i j}(t)$ a probabilidade da CMTC passar do estado $i$ para o estado $j$ depois de um tempo $t$, define-se a matriz de probabilidade de transição da CMTC como

$$
p(t)=\left\{p_{i j}(t)\right\}_{i j \in S \times S}
$$

$\operatorname{com} p(0)=I$ (matriz identidade).

Seja $\nu_{i}$ a taxa de saída do estado $i$. Definimos $q_{i j}=\nu_{i} p_{i j}$ como sendo a taxa com a qual a CMTC passa do estado $i$ para o estado $j$, com $i \neq j$. Podemos representar matematicamente uma CMTC por meio da sua matriz de taxas de transição $Q$, também chamado gerador infinitesimal, onde cada elemento $Q_{i j}, \forall i, j \in S$, está definido como:

$$
Q_{i j}= \begin{cases}q_{i j}, & \text { se } i \neq j \\ -\sum_{k \neq i} Q_{i k}, & \text { c.c. }\end{cases}
$$

Uma propriedade importante a se verificar é se a CMTC é ergódica. Frequentemente a probabilidade da CMTC estar no estado $j$ no tempo $t$ converge a um valor limite, independente do estado inicial. Seja este limite:

$$
\pi_{j}=\lim _{t \rightarrow \infty} p_{i j}(t)
$$

onde, para se verificar a existência desse limite, é suficiente que:

1. Todos os estados da CMTC se comuniquem, ou seja, para todo $i, j \in S$, a probabilidade de estar no estado $j$ iniciando no estado $i$, é positiva.

2. Iniciando em qualquer estado, o tempo médio para voltar nesse estado é finito, então a CMTC é recorrente positiva.

Se essas duas condições forem verificadas, $\pi_{j}$ existe e a CMTC é ergódica.

Ao longo deste trabalho, sempre que for usado o conceito de CMTC, tratar-se-á de uma CMTC ergódica, salvo seja indicado o contrário. 


\subsubsection{Análise de Regime Estacionário}

O limite $\pi_{j}$ é também conhecido como a probabilidade estacionária, já que, se o estado inicial é escolhido de acordo com a distribuição $\left\{\pi_{j}: j \in S\right\}$, então a probabilidade de estar no estado $j$ é $\pi_{j}$, para qualquer tempo $t$. A distribuição $\pi$ é a única solução para as equações lineares:

$$
\begin{gathered}
\pi_{j}=\sum_{k \in S} q_{k j} \pi_{k}, j \in S \\
\sum_{j \in S} \pi_{j}=1
\end{gathered}
$$

Onde 2.1 são equações de equilíbrio local e 2.2 é a equação de normalização, que garante que a solução $\pi$ seja única. Também 2.1 é equivalente a:

$$
\pi Q=0
$$

As equações de equilíbrio local expressam, no regime estacionário do sistema, que a taxa de saída de $j$ é igual à taxa de entrada em $j$, para todo $j \in S$.

\subsubsection{Análise Transiente}

Para a análise do sistema em tempos determinados é necessário observar o comportamento das probabilidades de transição, ao invés de estudar o estado estacionário. As equações de evolução para $p(t)$ dadas por $\frac{d}{d t} p(t)=Q p(t)=p(t) Q$, são chamadas de equações de Kolgomorov. A primeira igualdade produz:

$$
\frac{d}{d t} p_{i j}(t)=-q_{i} p_{i i}(t)+\sum_{k \neq i} q_{i k} p_{k j}(t)
$$

chamada a equação de Kolgomorov regressiva, que representa o fluxo que sai do estado $i$. Pode ser visto como o condicionamento de $X_{t+h}$ em $X_{h}$, fazendo $t \rightarrow 0$.

Analogamente, a segunda igualdade é equivalente a:

$$
\frac{d}{d t} p_{i j}(t)=-q_{i} p i i(t)+\sum_{k \neq i} p_{i k}(t) q_{k j}
$$

chamada equação de Kolgomorov progressiva, em termos do fluxo que entra no estado $i$. Isso, denota também o condicionamento de $X_{t+h}$ em $X_{t}$, com $h \rightarrow 0$.

\subsection{Ferramentas para modelagem analítica}

Várias técnicas foram criadas para serem utilizadas na modelagem de workflows como definido por Braghetto em [Bra11]. Algumas delas são usadas mais como ferramentas visuais, isto devido ao uso de gráficos e diagramas, o que faz com que, normalmente, elas sejam mais intuitivas para os usuários. Porém, elas sofrem de falta de formalismo na parte semântica, posto que muitas vezes elas dependem da área na qual estão sendo usadas, as características do processo e da interpretação que o projetista faz da técnica.

Por outro lado, também existem técnicas com semântica formal para modelagem de workflows. Algumas são mais básicas, como as máquinas de estado finitas e os sistemas de transição rotulados, que não têm expressividade suficiente para representar algumas características importantes 
dos workflows (como a ideia de concorrência), além de serem muito difíceis de se manipular para sistemas com um espaço de estados grande. Mas há outras técnicas mais expressivas e que conseguem representar concorrência, como é o caso das Redes de Petri, os Statecharts, as Álgebras de Processos e as Redes de Autômatos Estocásticos. As duas primeiras são mais visuais (gráficas) e portanto são mais usadas na modelagem de workflows. As Redes de Petri estão mais orientadas a fluxo de objetos, também chamados de tokens, e a transições. A técnica dos Statecharts está mais focada nos estados e transições entre estados [Har87, WW97, SM09]. Já as Álgebras de Processos têm uma representação textual e são descritas por meio de predicados, o que torna muitas vezes sua manipulação menos intuitiva; entretanto, sua notação é bem mais concisa. Por outro lado, as Redes de Autômatos Estocásticos considera que sistemas distribuídos e paralelos como uma coleção de componentes ou subsistemas, cada um deles sendo um autômato e por meio da interação entre eles é possível determinar o estado do sistema [Pla85].

Existem também técnicas mais recentes, muitas delas baseadas em alguma das técnicas formais mencionadas anteriormente. Como exemplo, pode-se citar os Diagramas de Decisão Binária Ordenados (do inglês Ordered Binary Decision Diagrams, OBDD, Helbig e Kelb 1994), que são representações obtidas a partir dos Statecharts [HK94].

Neste trabalho é importante e conveniente escolhermos uma técnica de modelagem de workflows formal, posto que o objetivo é usar modelagem analítica nos sistemas. Portanto, faz-se viável e relevante o uso de técnicas de modelagem amplamente utilizadas, como é o caso das Redes de Petri. Aliás, existem ferramentas que permitem manipular e aplicar as Redes de Petri, o que facilita o seu uso na modelagem de workflows, como por exemplo o GreatSPN [CFGR95, $\mathrm{BBC}^{+}$09] ou a ferramenta MARCIE [HRS13], entre outras. Além disso, algumas dessas ferramentas também permitem a análise dos modelos de rede criados nelas. Contudo, é importante destacar que não é comum encontrar ferramentas que se dedicam a fazer, mais especificamente, análise de desempenho preditiva. Além disso, algumas delas estão focadas em domínios de aplicação específicos.

\subsubsection{Redes de Petri}

As Redes de Petri (RPs) são modelos abstratos formais que foram desenvolvidos como uma ferramenta para a descrição e análise do fluxo de informação e controle em sistemas [Bal07]. Os sistemas para os quais as RPs são usadas normalmente têm como características principais a presença de concorrência e conflitos. Nas RPs, o estado do sistema pode ser obtido a partir da combinação de variáveis de estado local, que permitem uma representação direta da concorrência, causalidade e independência.

Os modelos de Rede de Petri têm como principal vantagem sua natureza gráfica, tornando-os mais intuitivos para se modelar sistemas, por fornecerem uma visão mais clara dos aspectos dos sistemas reais modelados. Além disso, as RPs são muito utilizadas por serem modelos formais e, consequentemente, possuírem técnicas matemáticas para estudá-las [vdA98, Bra11, VDAVH04].

Um modelo de Rede de Petri é constituído por duas partes: a estrutura da rede e a marcação. A estrutura da rede é um grafo bipartido dirigido, onde as duas partições são formadas por lugares e transições, ou seja um nó pode ser apenas de um desses tipos. Por esse motivo, existem também dois tipos de arestas, as que ligam lugares a transições, chamadas arestas de entrada, e as arestas de saída são as que ligam transições a lugares. Graficamente, os lugares são representados por círculos e as transições por retângulos, como mostrado na Figura 2.2. Os lugares representam variáveis de estado no sistema e as transições representam ações que induzem mudança de estados. 


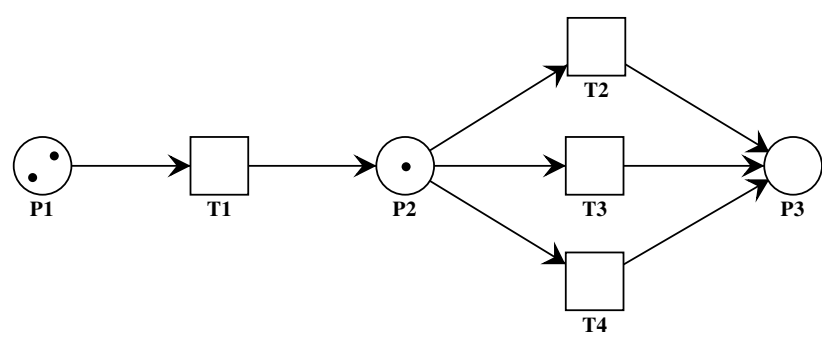

Figura 2.2: Modelo simples de uma Rede de Petri, onde os lugares são P1, P2 e P3 e as transições são T1, T2, T3 e T4. A marca da Rede de Petri é $\{2,1,0\}$

A marcação da Rede de Petri é a atribuição de fichas aos lugares, que representam o valor do estado. É por meio dessas fichas que podemos expressar dinâmica na Rede de Petri, ou seja, o seu comportamento é dado pela evolução da sua marcação, que tem as seguintes regras:

- Habilitação - uma transição pode acontecer (ser habilitada) quando os valores dos lugares de entrada cumprem as condições requeridas pelas arestas que os ligam. Normalmente essa condição é que cada lugar de entrada tenha pelo menos uma ficha.

- Disparo - o acontecimento de uma transição (disparo) muda os valores dos lugares adjacentes (de entrada e saída) de acordo com a condição de cada aresta. Geralmente a condições fazem com que cada lugar de entrada perca uma ficha e cada lugar de saída ganhe uma.

Na sequência, temos a definição formal das Redes de Petri [Bal07, LZJ16].

Definição 2.7.1. Uma Rede de Petri é uma tupla $\left(P, T, F, W, \mathbf{m}_{0}\right)$, onde:

- $P=\left(p_{1}, p_{2}, \ldots, p_{P}\right)$ é o conjunto de lugares,

- $T=\left(t_{1}, t_{2}, \ldots, t_{T}\right)$ é o conjunto de transições,

- $F \subseteq(P \times T) \cup(T \times P)$ é o conjunto de arestas,

- $W: F \rightarrow \mathbb{N}$ é a função de peso das arestas,

- $\mathbf{m}_{0}=\left(m_{01}, m_{02}, \ldots, m_{0 P}\right)$ é a marcação inicial.

Logo, seja o nó $v \in(P \cup T)$, seus conjuntos de entrada e saída podem ser definidos respectivamente como: $\bullet v=\{u \mid\langle u, v\rangle \in F\}$ e $v \bullet=\{u \mid\langle v, u\rangle \in F\}$. Dessa forma, as duas regras da evolução da marcação podem ser expressas formalmente como mostrado a seguir:

- Uma transição $t$ está habilitada em uma marcação $m$ se, e somente se,

$$
\forall p \in \bullet, m(p) \geqslant W(<p, t>)
$$

denotado por $m[t\rangle$. Então, $\neg m[t\rangle$ indica que $t$ não está habilitada em $m$. Além disso, denotamos por $E(m)$ o conjunto de todas as transições habilitadas na marcação $m$.

- O disparo de $t$, habilitada em $m$, gera a marcação $m^{\prime}$, tal que: $m^{\prime}=m+O(t)-I(t)$, com

$$
\begin{aligned}
O(t) & =\left(W\left(<t, p_{1}>\right), W\left(<t, p_{2}>\right), \ldots, W\left(<t, p_{L}>\right)\right) \\
I(t) & =\left(W\left(<p_{1}, t>\right), W\left(<p_{2}, t>\right), \ldots, W\left(<p_{L}, t>\right)\right)
\end{aligned}
$$

Isto indica que $m^{\prime}$ é diretamente alcançavél a partir de $m$ por meio de $t$, denotado por $m[t\rangle m^{\prime}$. 
Para $m$, pode-se definir também o conjunto de todas as marcações alcançáveis $R M(m)$, onde, $m^{\prime} \in R M(m)$ se, e somente se, existe uma sequência de transições $\left\{t_{(1)}, \ldots, t_{(j)}\right\}$ e uma sequência de marcações $\left\{m_{(1)}, \ldots, m_{(j+1)}\right\}$, sendo que $m_{(1)}=m$ e $m_{(j+1)}=m^{\prime}$, tal que, $m_{(i)}\left[t_{(i)}\right\rangle m_{(i+1)}$, com $1 \leqslant i \leqslant j$. A partir da marcação inicial $m_{0}$, podemos definir $R M\left(m_{0}\right)$ como sendo o espaço de estados da Rede de Petri. Quando tem-se a informação da sequência de transições para atingir cada marcação, denomina-se ao conjunto $R M\left(m_{0}\right)$ como Grafo de Alcançabilidade, onde cada nó representa um estado (marcação) alcançável, e existe uma aresta entre dois nós $m_{i}$ e $m_{j}$ se $m_{j}$ é diretamente alcançável de $m_{i}$, ou seja $\exists t_{x}$ tal que $m_{i}\left[t_{x}\right\rangle m_{j}$, que em realidade gera um multigrafo.

Algumas propriedades importantes das Redes de Petri, para avaliar o sistema representado, são:

- Alcançabilidade e Reversibilidade - Uma marcação $m^{\prime}$ é alcançável da marcação $m$ se $m^{\prime} \in$ $R M(m)$. Dessa forma, para qualquer marcação alcançável da marcação inicial $m_{0}$, a Rede de Petri é reversível se é possível voltar na marcação inicial.

- Vivacidade - Um transição $t$ é viva se e somente se, para cada $m \in R M\left(m_{0}\right)$, existe uma marcação $m^{\prime} \in R M(m)$, tal que $t \in E\left(m^{\prime}\right)$. Assim, a Rede de Petri é viva se todas as transições são vivas. Se pelo menos uma transição é viva, então a Rede de Petri não tem ponto morto (deadlock), ou seja, não existe um estado da Rede de Petri no qual nenhuma transição esteja habilitada. De outro lado, se todas as transições são vivas, a Rede de Petri não tem livelock, isto é, não existe um subconjunto de transições dos quais não consiga sair.

- Delimitação - Um lugar $p$ é $k$-delimitado se e somente se, para cada marcação alcançável, o número de fichas em $p$ é menor igual a $k$. Portanto a Rede de Petri é $k$-delimitada se $\forall p \in P$, $p$ é $k$-delimitado. No caso específico de $k=1$, a Rede de Petri é chamada segura. Uma consequência importante da delimitação é a finitude do espaço de estados, assim, numa Rede de Petri $k$-delimitada com $N$ lugares, o número de estados é menor ou igual a $(k+1)^{N}$

Diferentes extensões tem sido aplicadas nas Redes de Petri, com a finalidade de aumentar a representatividade de diversos sistemas [CR83]. Para este trabalho, uma das extensões mais relevantes é a de associação de tempos aos componentes das redes. Mais especificamente, em nosso caso será de maior utilidade a temporização das transições (tempo de disparo da transição), pois elas descrevem a realização de uma tarefa, portanto, o tempo da transição indica o tempo que a tarefa demora para ser realizada.

\subsubsection{Redes de Petri Estocásticas Generalizadas}

As Redes de Petri Estocásticas (RPE) [Mol82] são aquelas em que os tempos de disparo são especificados por variáveis aleatórias. Uma RPE Generalizada (RPEG) é aquela que tem dois tipos de transições: as temporizadas e as imediatas [CMBC93]. As transições temporizadas descrevem a execução de atividades demoradas. Elas são definidas por meio da distribuição exponencial negativa, ou seja, o tempo de disparo de uma transição é uma variável aleatória com distribuição exponencial negativa. O uso dessa distribuição permite o mapeamento da RPEG para Cadeia de Markov de Tempo Continuo (CMTC). De outro lado, transições imediatas representam atividades instantâneas, usadas, por exemplo, para verificar condições lógicas. Denotaremos por $w_{k}$ a taxa de disparo de uma transição temporizada $t_{k}$.

Além da RPEG possuir transições temporizadas e temporais, é necessário verificar que ela seja $k$-delimitada, isto garante o isomorfismo com uma CMTC. Para obter a CMTC isomórfica à RPEG, se aplica os seguintes passos: 
1. O espaço de estados $S=\left\{s_{i}\right\}$ da CMTC, corresponde ao conjunto $R M\left(m_{0}\right)$ da RPEG, então temos a correspondência $m_{i} \leftrightarrow s_{i}$.

2. Sejam os estados $s_{i}, s_{j} \in S$, a taxa da transição de $s_{i}$ para $s_{j}$ é definida como: a soma das taxas de disparo das transições habilitadas em $m_{i}, t \in E\left(m_{i}\right)$, tal que $m_{i}[t\rangle m_{j}$, denominaremos esse conjunto como $H_{j}\left(m_{i}\right)$.

Então a matriz infinitesimal $Q$ da CMTC está definida por:

$$
Q_{i j}= \begin{cases}\sum_{t_{k} \in H_{j}\left(m_{i}\right)} w_{k}, & \text { se } i \neq j \\ -\sum_{t_{k} \in H\left(m_{i}\right)} w_{k}, & \text { c.c. }\end{cases}
$$

Dado que nas CMTCs existe uma única distribuição estacionária ( $\pi$ ) se a cadeia for ergódica, é necessário garantir que a rede produza este tipo de cadeia. Uma condição necessária e suficiente é que a RPEG seja reversível (onde o estado inicial $m_{0}$ é chamado estado origem) ${ }^{2}$. É por meio da distribuição estacionária $\pi$ que podemos calcular índices de desempenho do sistema modelado na RPEG.

Na Figura 2.3 temos uma Rede de Petri reversível, isto é fácil de verificar a partir do grafo de alcançabilidade mostrado na Figura 2.4, onde a marcação inicial é $M 1$ (só o lugar $P 0$ tem uma ficha e os outros lugares não tem fichas), aqui podemos observar que a partir de qualquer marcação é possível voltar na marcação inicial.

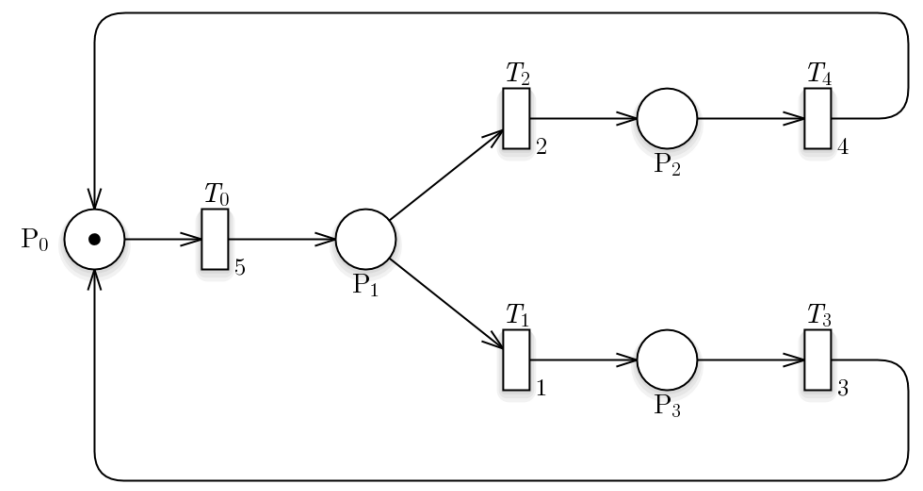

Figura 2.3: Rede de Petri reversível

\subsubsection{2 Índices de desempenho da RPEG}

Para realizar a análise quantitativa do comportamento de uma RPEG, descrita em índices de desempenho, é necessário o cálculo da distribuição estacionária $\pi$ da CMTC isomórfica à rede. Para os índices de desempenho são definidas funções de indexação sobre as marcações da rede [Bal07]. Assim, seja o índice de desempenho $R$ e sua função de indexação $r(m)$, o valor médio de $R$ pode ser calculado por

$$
\mathbb{E}[R]=\sum_{m_{i} \in R M\left(m_{0}\right)} r\left(m_{i}\right) \pi_{i}
$$

Por exemplo, seja $r(m)=n$ se $m\left(p_{j}\right)=n$, pela Equação 2.6 obtemos o valor médio de fichas no lugar $p_{j}$. Por outro lado, quando $r(m)=w_{j}$ se $t_{j} \in E(m)$, a Equação 2.6 fornecerá a média do

\footnotetext{
${ }^{2}$ No restante do texto, quando uma RPEG é usada, assume-se que ela é ergódica, a menos que seja dito o contrário.
} 


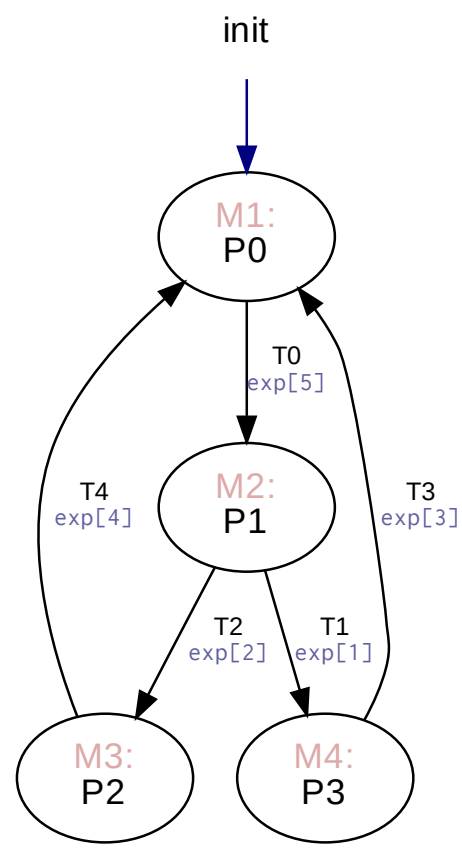

Figura 2.4: Grafo de alcançabilidade da Rede de Petri em 2.3

número de disparos de $t_{j}$ por unidade de tempo, i.e., o rendimento da transição.

É possível também utilizar a análise transiente para calcular os índices de desempenho, nesse caso são utilizadas as equações de Kolgomorov descritas na Seção 2.6.

No próximo capítulo serão abordadas estruturas básicas de workflows e suas modelagens por meio das Redes de Petri, descritas neste capítulo, permitindo uma comparação mais objetiva dos métodos. 


\section{Capítulo 3}

\section{Modelagem markoviana de workflows de negócio de nu- merosas instâncias}

As Redes de Petri são bastante usadas no gerenciamento de processos de negócio, por serem um formalismo que permite a modelagem e análise do processo de uma forma intuitiva [vdA04]. Este capítulo descreve a modelagem de workflows por meio de RPEGs. Como definido na Seção 2.7, esta técnica se baseia em processos Markovianos, que têm sido muito utilizados na área de modelagem de sistemas dinâmicos, tanto na análise qualitativa quanto na análise quantitativa.

Neste capítulo, serão implementadas estruturas básicas de workflows que são comumente encontradas nos processos de negócio. A partir dessas estruturas podem ser construídos modelos de workflow mais complexos, que são os que geralmente se encontram no mundo real. Uma vez definida a RPEG que representa o workflow, somos capazes de realizar a análise de desempenho do sistema.

Uma das vantagens do uso das Redes de Petri é o fato de existir ferramentas dedicadas à sua análise formal. Neste trabalho usaremos a ferramenta GreatSPN [CFGR95, BBC ${ }^{+}$09], que permite a construção gráfica das Redes de Petri e faz a análise quantitativa de forma automatizada, por meio da ferramenta de análise MC4CSL ${ }^{\mathrm{TA}}$ [AD13].

Por último, discutiremos o problema da explosão do espaço de estados, que apresenta-se nos modelos Markovianos, pelo fato de examinarem (quase) todos os estados possíveis do sistema, o que é inviável para workflows com muitas instâncias.

\subsection{Modelagem Markoviana de Estruturas Básicas de Workflows}

Considerando a perspectiva de controle de fluxo, foram mostrados na Seção 2.1 alguns tipos de comportamento frequentes em workflows, enquanto que na Seção 2.2 foram mostrados os componentes da notação BPMN que modelam esses tipos de comportamento. Também introduziu-se regras para se ter um workflow bem-definido e uma notação para a declaração dos requisitos de recursos de cada uma das suas atividades (definições 2.3.1 e 2.3.2 ). Agora, descreveremos a forma de se derivar as RPEGs correspondentes a workflows bem-definidos e com anotações de recursos.

Primeiro, cria-se um lugar inicial na RPEG onde o número de fichas deve corresponder ao número total de instâncias do workflow que se quer modelar. Depois, deve-se criar um lugar para cada tipo de recurso usado no workflow, cada um deles com um número de fichas equivalente ao número total do recurso correspondente.

\subsubsection{Mapeamento de uma Atividade}

Para toda atividade, primeiro precisamos alocar os recursos necessários para sua execução. Uma vez alocado(s) o(s) recurso(s), a atividade é executada e, assim que for concluída, os recursos 
alocados são liberados. Seja a atividade $A$, cujos requisitos estão especificados pela proposição $P_{A}=\left(P_{A 1} \vee \cdots \vee P_{A n}\right)$, onde cada proposição $P_{A n}, 1 \leqslant i \leqslant n$, se verdadeira, permite que a atividade seja executada.

Assim, para o mapeamento de $A$, criamos transições de alocação de recursos para cada $P_{A i}$, denotadas por $A l c_{P_{A i}}$, cuja taxa de alocação toma o valor do parâmetro $r$, que indica um valor experimental para a alocação de recursos. A execução da atividade propriamente dita é mapeada por meio de transições de execução, denotadas como $\operatorname{Exec}_{P_{A i}}$, cuja taxa é dada pelo recurso mais lento especificado em $P_{A i}$, ou seja, aquele que mais demora para executar a atividade. $O$ valor da taxa de execução é a razão entre a capacidade de trabalho do recurso e a quantidade de trabalho da atividade a ser executada pelo recurso.

Suponhamos uma atividade $A$, com requisito $P_{A}=\left(\left(\left[R_{1}, a\right] \wedge\left[R_{2}, b\right]\right) \vee\left[R_{1}, c\right]\right)$. Na Figura 3.1 mostra-se o mapeamento em RPEG da atividade $A$, onde $t R_{1}$ representa a taxa de execução da atividade $A$ utilizando o recurso $R_{1}$ (razão entre a capacidade de trabalho de $R_{1}$ e quantidade de trabalho requerida para a atividade $A$, neste caso $c$ ) e tmin representa o menor valor entre as taxas de execução de $A$ nos recursos $R_{1}$ e $R_{2}$; é importante observar que tmin representa o recurso mais lento por causa da distribuição exponencial.

Na RPGE da Figura 3.1 uma ficha no lugar $P_{0}$ representa uma instância prestes a executar a atividade $A$. A primeira etapa é alocar os recursos requisitados pela atividade, assim, a transição $A l c P A_{1}$ representa a alocação dos recursos $R_{1}$ e $R_{2}$, ou seja, ela só será habilitada se existir pelo menos uma ficha em cada um dos lugares $R_{1}$ e $R_{2}$, então uma ficha em $P_{1}$ representa uma instância que alocou os dois recursos. Por outro lado, já que a atividade $A$ pode ser executada de duas formas diferentes, a transição $A l c P A_{2}$ modela a alocação do recurso $R_{1}$, assim, $P_{2}$ representa as instâncias que alocaram $R_{1}$. Finalmente, em $P_{3}$ estão as fichas que executaram efetivamente a atividade, seja pela transição $\operatorname{ExecP} A_{1}$ ou $\operatorname{ExecP} A_{2}$, com suas respetivas taxas que dependem dos recursos previamente alocados.

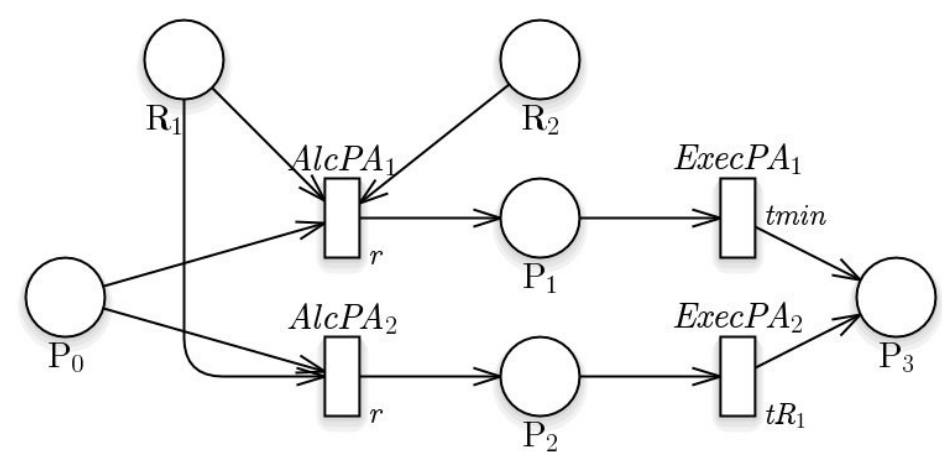

Figura 3.1: Mapeamento em $R P G E$ de uma atividade $A$ com requisito $P_{A}=\left(\left(\left[R_{1}, a\right] \wedge\left[R_{2}, b\right]\right) \vee\left[R_{1}, c\right]\right)$

\begin{tabular}{l|l}
\hline Lugares & Transições \\
\hline$P_{0}:$ tarefa A está habilitada & $A l c P A_{1}:$ A aloca recursos $R_{1}$ e $R_{2} \operatorname{com}$ taxa $r$ \\
$P_{1}:$ tarefa A está sendo executada & $A l c P A_{2}:$ A aloca recurso $R_{1}$ com taxa $r$ \\
$P_{2}:$ tarefa A está sendo executada & $E x e c P A_{1}:$ A é executada com taxa $t$ min \\
$P_{3}:$ tarefa A foi executada & $E x e c P A_{2}:$ A é executada com taxa $t R_{1}$ \\
$R_{1}:$ recursos disponíveis $R_{1}$ & \\
$R_{2}:$ recursos disponíveis $R_{2}$ & \\
\hline
\end{tabular}

Tabela 3.1: Descrição dos componentes da RPEG na Figura 3.1. 


\subsubsection{Mapeamento de uma Sequência de Atividades}

Para uma estrutura sequencial, que considera a execução de atividades uma após a outra, o mapeamento em RPEG compreende a modelagem individual de cada uma das atividades que compõem a sequência.

Por exemplo, considere o workflow da Figura 3.2 e suponha que temos apenas o recurso $R_{1}=$ $\left(R_{1}, N R_{1}, c R_{1}\right)$ e que os requisitos são: para a atividade $A$ temos $P_{A}=\left(\left[R_{1}, q t A\right]\right)$ e para a atividade $B$ temos $P_{B}=\left(\left[R_{1}, q t B\right]\right)$. O modelo em RPEG desse workflow é mostrado na Figura 3.3, onde $A R_{1}=c R_{1} / q t A$ e $B R_{1}=c R_{1} / q t B$.

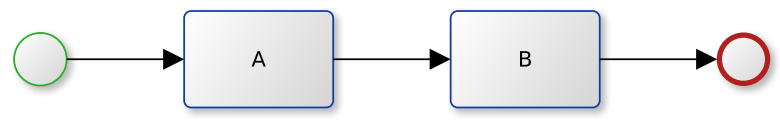

Figura 3.2: Modelo BPMN com estrutura sequencial de atividades.

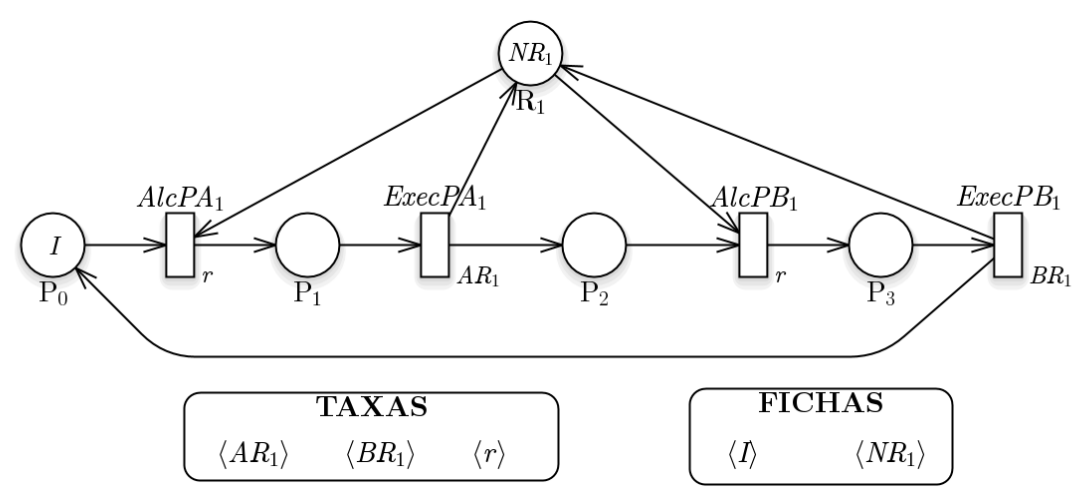

Figura 3.3: RPEG da estrutura sequencial mostrada na Figura 3.2.

\begin{tabular}{l|l}
\hline Lugares & Transições \\
\hline$P_{0}:$ tarefa A está habilitada & $A l c P A_{1}:$ A aloca recurso $R_{1}$ com taxa $r$ \\
$P_{1}:$ tarefa A está sendo executada & $E x e c P A_{1}:$ A é executada com taxa $A R_{1}$ \\
$P_{2}:$ tarefa B está habilitada & $A l c P B_{1}:$ B aloca recurso $R_{1}$ com taxa $r$ \\
$P_{3}:$ tarefa B está sendo executada & $\operatorname{Exec} P B_{1}:$ B é executada com taxa $B R_{1}$ \\
$R_{1}:$ recursos disponíveis $R_{1}$ & \\
\hline
\end{tabular}

Tabela 3.2: Descrição dos componentes da RPEG na Figura 3.3.

\subsubsection{Mapeamento de uma Escolha}

Para uma estrutura de escolha, formada pelos componentes XOR-split e XOR-join, cria-se uma ramificação (representada por uma transição) para cada uma das possíveis linhas de execução, onde a taxa de execução de uma linha é proporcional à probabilidade dela ser executada. Por último, cada uma dessas ramificações deve se juntar num único lugar da RPEG, que representa o componente XOR-join.

A Figura 3.4 mostra um workflow com uma estrutura básica de escolha com duas possíveis linhas de execução, cada uma composta por apenas uma atividade ( $A$ e $B$ ), cujas probabilidades são $\operatorname{pr} 1$ e $\operatorname{pr} 2$ respectivamente. Suponha que as atividades $A$ e $B$ são especificadas da mesma forma que na estrutura de sequência da Figura 3.2. Portanto, temos a RPEG da Figura 3.5 como sendo o mapeamento da estrutura de escolha do workflow modelado na Figura 3.4.

Podemos ver que a transição temporizada $X$ or $_{1}$ representa a linha de execução da atividade $A$, com taxa de execução $p r 1$ proporcional à probabilidade $\operatorname{pr} 1$ no modelo BPMN; da mesma forma, 
$X o r_{2}$ é a transição para executar a linha da atividade $B$. Também, vemos que as duas ramificações se unem em uma única linha de execução, no caso, $P_{0}$ representa a união das ramificações do bloco de escolha.

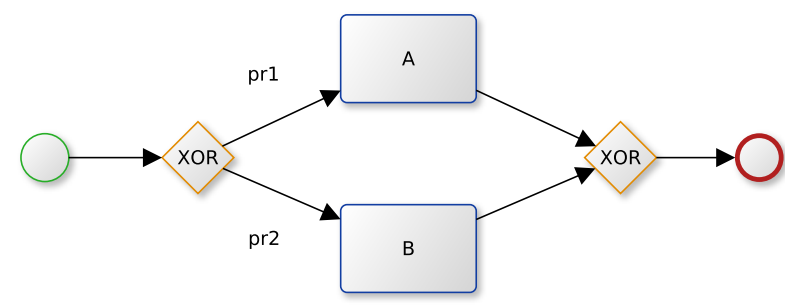

Figura 3.4: Modelo BPMN com estrutura de escolha

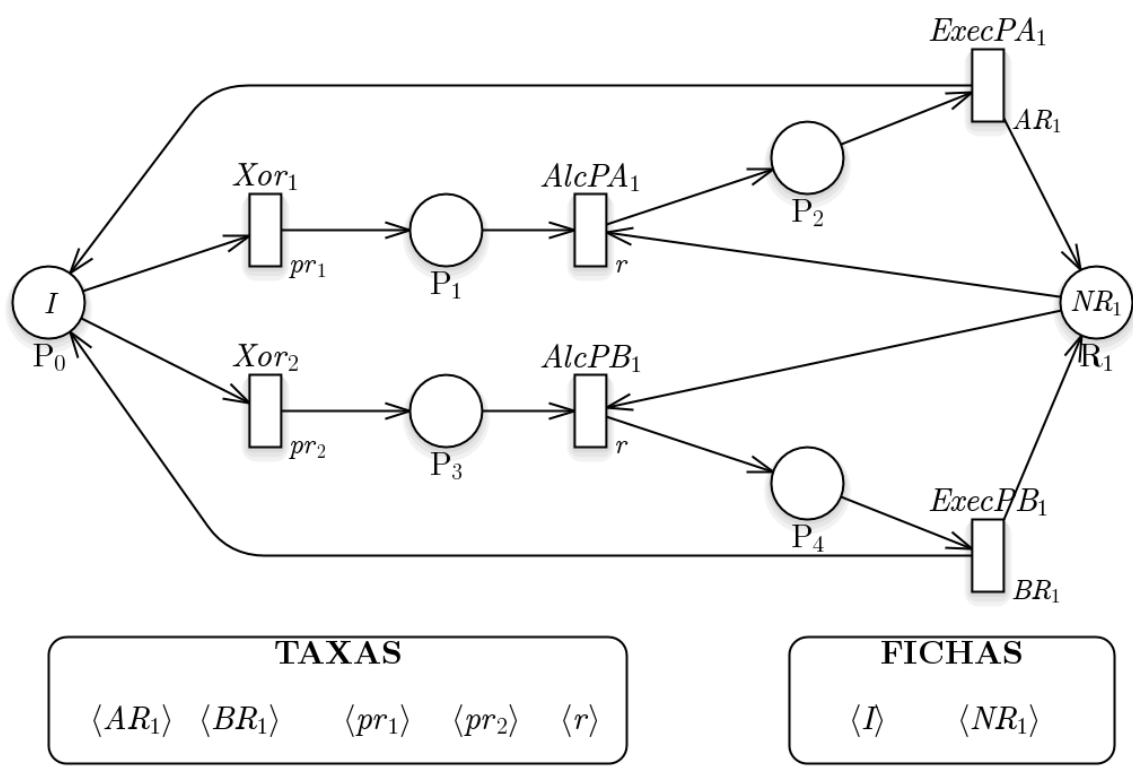

Figura 3.5: RPEG da estrutura de escolha mostrada na Figura 3.4

\begin{tabular}{|c|c|}
\hline Lugares & Transições \\
\hline$P_{0}$ : escolha exclusiva & $X o r_{1}$ : A escolhida com taxa $p r_{1}$ \\
\hline$P_{1}:$ tarefa A está habilitada & $\mathrm{Xor}_{2}:$ B escolhida com taxa $p r_{2}$ \\
\hline$P_{2}$ : tarefa A está sendo executada & $A l c P A_{1}$ : A aloca recurso $R_{1}$ com taxa $r$ \\
\hline$P_{3}:$ tarefa $\mathrm{B}$ está habilitada & $E x e c P A_{1}$ : A é executada com taxa $A R_{1}$ \\
\hline$P_{4}$ : tarefa $\mathrm{B}$ está sendo executada & $A l c P B_{1}$ : B aloca recurso $R_{1}$ com taxa $r$ \\
\hline$R_{1}:$ recursos disponíveis $R_{1}$ & $E x e c P B_{1}:$ B é executada com taxa $B R_{1}$ \\
\hline
\end{tabular}

Tabela 3.3: Descrição dos componentes da RPEG na Figura 3.5.

\subsubsection{Mapeamento do Paralelismo}

O paralelismo do workflow, definido no modelo BPMN pelos componentes AND-split e ANDjoin, pode ser mapeado na RPEG como um bloco de escolha onde as linhas de execução possíveis correspondem a cada possível ordenação dos fluxos de execução que compõem a estrutura paralela. Consideramos uma ordenação dos fluxos paralelos como sendo uma permutação desses fluxos. Estas ordenações (que executam os fluxos paralelos de forma sequencial) são equivalentes, quantitativamente falando, ao paralelismo devido à propriedade de perda de memória da distribuição exponencial. No Capítulo 4 detalhamos e verificamos esta propriedade importante nos modelos Markovianos. 
Como exemplo, no workflow da Figura 3.6 temos dois fluxos que são executados em paralelo, cada um deles composto por uma atividade, de forma que $A$ e $B$ possam ser executadas em paralelo. Sejam as especificações de recursos e requisitos iguais às mostrados para a estrutura sequencial e a de escolha. O mapeamento dessa estrutura de paralelismo é mostrado na RPEG da Figura 3.7.

Já que as duas atividades ( $A$ e $B$ ) são executadas em paralelo, na RPEG temos duas ordenações de execução: a primeira executa primeiro $A$ e depois $B$; por outro lado, a segunda ordenação executa $B$ e depois $A$.

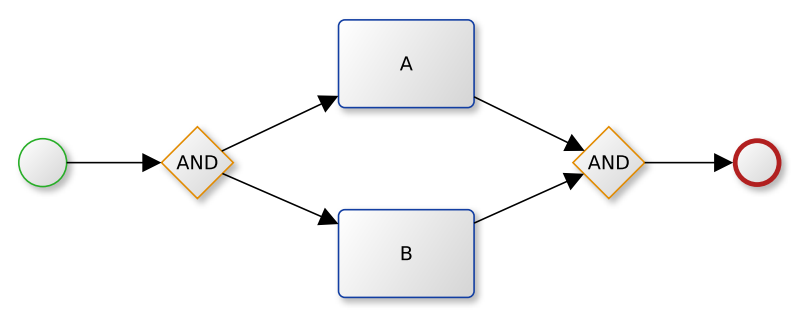

Figura 3.6: Modelo BPMN com estrutura paralela

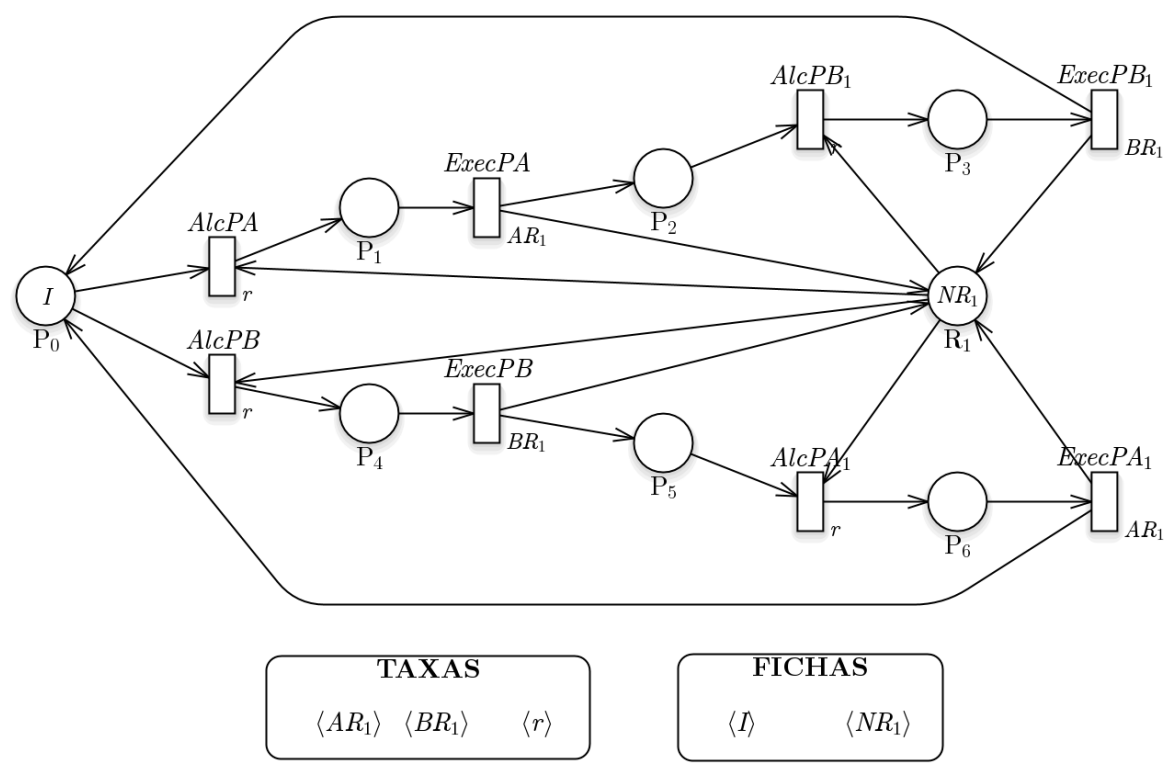

Figura 3.7: RPEG modelando a estrutura paralela mostrada na Figura 3.6

\begin{tabular}{l|l}
\hline Lugares & Transições \\
\hline$P_{0}:$ início do paralelismo & $A l c P A:$ A aloca recurso $R_{1}$ com taxa $r$ \\
$P_{1}:$ tarefa A está sendo executada & ExecPA: A é executada com taxa $A R_{1}$ \\
$P_{2}:$ tarefa B está habilitada & $A l c P B_{1}:$ B aloca recurso $R_{1}$ com taxa $r$ \\
$P_{3}:$ tarefa B está sendo executada & ExecPB $B_{1}:$ B é executada com taxa $B R_{1}$ \\
$P_{4}:$ tarefa B está sendo executada & $A l c P B: \mathrm{B}$ aloca recurso $R_{1}$ com taxa $r$ \\
$P_{5}:$ tarefa A está habilitada & ExecPB: B é executada com taxa $B R_{1}$ \\
$P_{6}:$ tarefa A está sendo executada & $A l c P A_{1}:$ A aloca recurso $R_{1}$ com taxa $r$ \\
$R_{1}:$ recursos disponíveis $R_{1}$ & ExecPA $A_{1}:$ A é executada com taxa $A R_{1}$ \\
\hline
\end{tabular}

Tabela 3.4: Descrição dos componentes da RPEG na Figura 3.7.

Tendo definido o mapeamento de estruturas básicas de workflows para Redes de Petri, é possível representar workflows que apresentem composições dessas estruturas, resultando em modelos mais complexos. Na Figura 3.8 apresentamos um workflow que apresenta as três estruturas básicas antes 
mencionadas em um único modelo.

O workflow da Figura 3.8 apresenta uma escolha, onde uma ramificação executa um paralelismo composto pelas atividades $A$ e $B$; já a outra ramificação deriva numa estrutura de sequência composta pelas atividades $C$ e $D$. Para o sistema temos apenas um recurso, requerido por todas as atividades. O mapeamento desse workflow em RPEG resultante é mostrado na Figura 3.9.

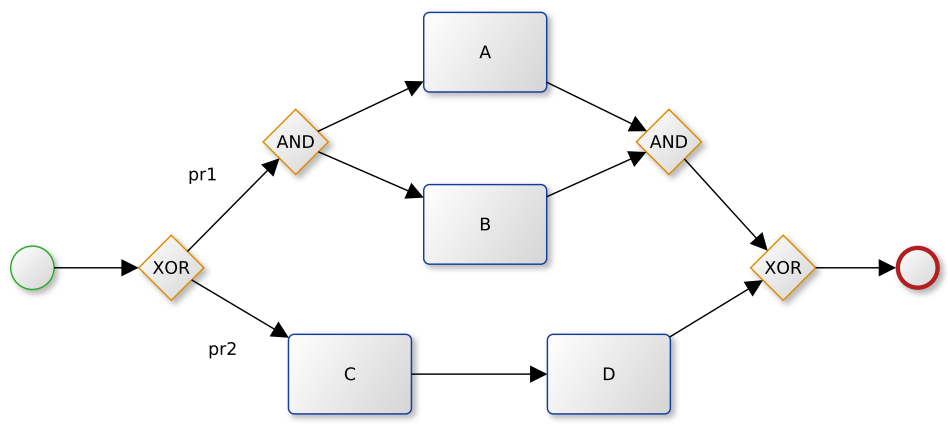

Figura 3.8: Modelo de workflow que apresenta a combinação das três estruturas básicas: sequência, escolha e paralelismo

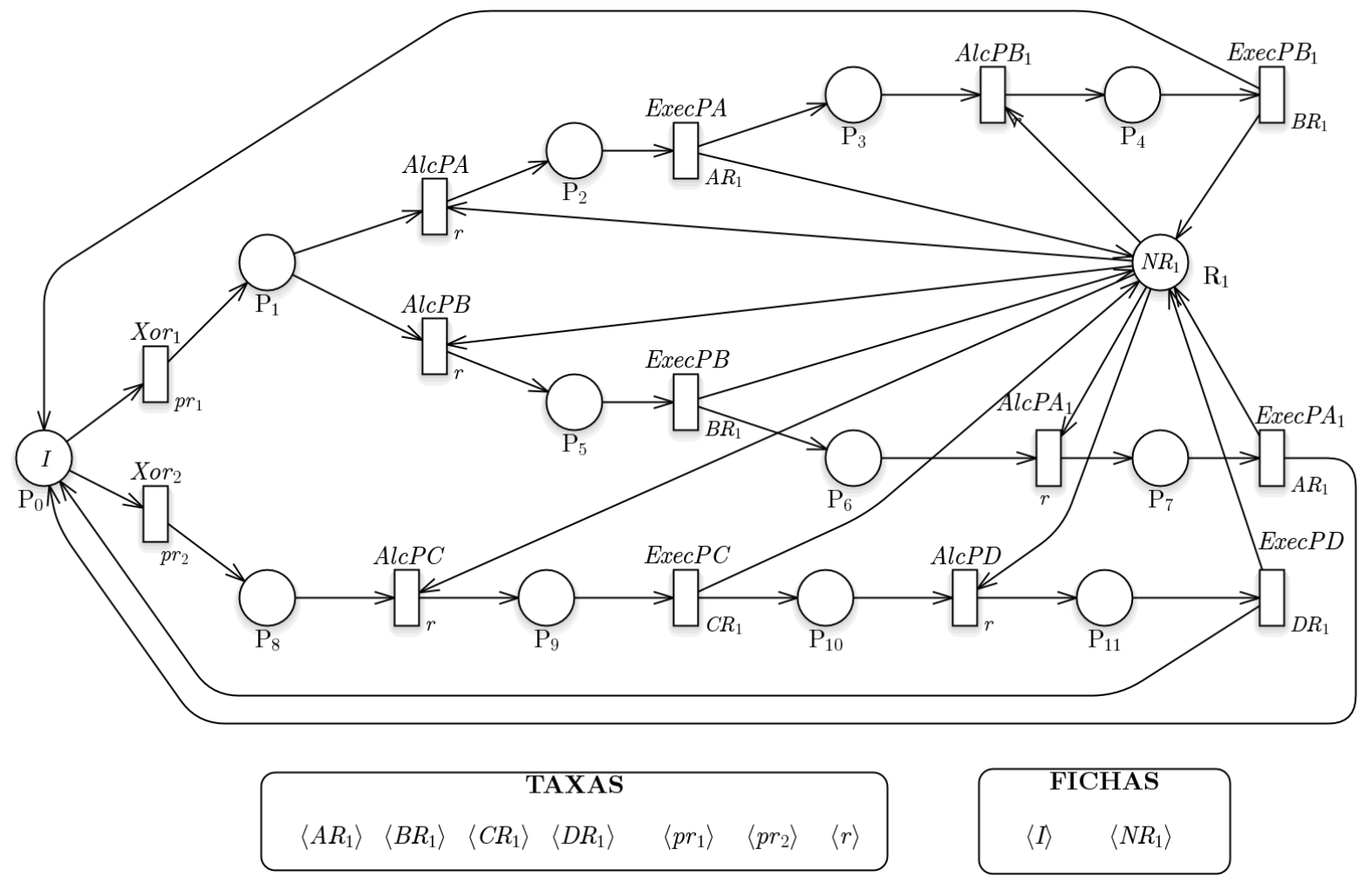

Figura 3.9: Modelagem por meio de RPEG do modelo de workflow mostrado na Figura 3.8

\subsection{Análise de Desempenho de Modelos Markovianos}

Uma vez que temos as RPEGs a partir dos modelos BPMN dos workflows, somos capazes de analisar o comportamento desses sistemas para poder extrair informação referente ao seu desempenho, isto é, via modelagem analítica, usamos técnicas matemáticas para analisar as CMTCs subjacentes aos modelos de RPEGs.

Como explicado na Seção 2.7, para definir a CTMC isomórfica a uma RPEG, é necessário gerar o grafo de alcançabilidade da rede.

Por exemplo, para a RPEG da Figura 3.3, considere a seguinte configuração: 
- Recurso $R_{1}=\left(R_{1}, 2,40\right)$;

- Requisitos:

- Atividade $A: P_{A}=\left(\left[R_{1}, 10\right]\right)$;

- Atividade $B: P_{B}=\left(\left[R_{1}, 20\right]\right)$;

- O número de fichas é: $I=1$ e $N R_{1}=2$;

- As taxas são: $A R_{1}=40 / 10, B R_{1}=40 / 20$ e $r=500 .^{1}$

Sob essa configuração é obtido o grafo de alcançabilidade da Figura 3.10, onde podemos observar que o sistema tem 4 possíveis estados e 4 transições entre estados. Se tivéssemos 2 instâncias $(I=2)$, o sistema teria 10 estados e 16 transições. Na Tabela 3.5 se mostra o tamanho do sistema para diferentes configurações.

Assim que o grafo de alcançabilidade é gerado e, portanto, também temos a CMTC isomórfica subjacente, é possível calcular medidas de desempenho do sistema. Para tal propósito é necessário calcular a distribuição estacionária da CMTC para fazer a análise estacionária, e depois definir as funções de indexação (valor médio de fichas por lugar, número de disparos das transições e outras), dessa forma, podemos calcular as medidas de desempenho por meio da Equação 2.6.

Por outro lado, também podemos usar as Equações de Kolgomorov 2.3 e 2.4, para realizar a análise transiente do sistema e calcular medidas de desempenho para um determinado tempo.

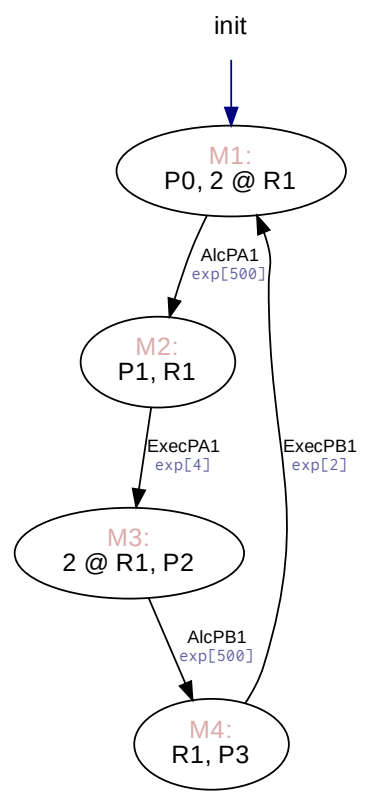

Figura 3.10: Grafo de alcançabilidade da RPEG mostrada na Figura 3.3 quando existe só uma ficha em $P_{0}$ (número de instâncias).

onde:

\footnotetext{
${ }^{1}$ A taxa de alocação $r$ normalmente terá o valor de 500 ao longo deste trabalho, este é um valor que foi atingido depois de ter realizado vários testes com outros valores, mostrando menor impacto nos modelos Markovianos e nos modelos determinísticos, já que valores menores causam divergência das aproximações e valores maiores obtêm soluções com mais tempo de computo.
} 
- $\left(M 1: P_{0}, 2 @ R_{1}\right)$ - é o estado inicial, com 1 instância (ficha) em $P_{0}$ e 2 unidades de recurso $R_{1}$ disponíveis;

- (M2: $\left.P_{1}, R_{1}\right)$ - estado com 1 instância em $P_{1}$ e 1 unidade de recurso $R_{1}$ disponível;

- $\left(M 3: 2 @ R_{1}, P_{2}\right)$ - estado com 2 unidades de recurso $R_{1}$ disponíveis e 1 instância em $P_{2}$;

- $\left(M 4: R_{1}, P_{3}\right)$ - estado com 1 unidade de recurso $R_{1}$ disponível e 1 instância em $P_{3}$;

Seja $G$ o grafo de alcançabilidade da Figura 3.10. Logo, o gerador infinitesimal da CMTC subjacente a $G$ é:

$$
Q=\left(\begin{array}{cccc}
-500 & 500 & 0 & 0 \\
0 & -4 & 4 & 0 \\
0 & 0 & -500 & 500 \\
2 & 0 & 0 & -2
\end{array}\right)
$$

onde o estado estacionário é:

$$
\pi=\{0.00265252,0.331565,0.00265252,0.66313\}
$$

Continuando com o exemplo, suponhamos que queremos calcular o número médio de fichas do lugar $P_{0}$. Então, precisamos analisar cada marcação e ver o número de fichas em $P_{0}$ para essa marcação e multiplicar pelo valor médio da marcação (no estado estacionário). Seja a função $r\left(M_{i}\right)$, que representa o número de fichas em $P_{0}$ para a marcação $M_{i} \in G$, temos que o valor médio de fichas em $P_{0}$ será

$$
\begin{aligned}
\mathbb{E}\left(P_{0}\right) & =\sum_{M_{i} \in G} r\left(M_{i}\right) \pi_{i} \\
& =1 \times \pi_{1}+0 \times \pi_{2}+0 \times \pi_{3}+0 \times \pi_{4} \\
& =\pi_{1}=0.00265252
\end{aligned}
$$

Dessa forma podemos calcular o valor médio de fichas em cada lugar da RPEG.

Também, para calcular o rendimento das transições (média de disparos da transição por unidade de tempo), o processo é semelhante. Seja $r\left(M_{i}\right)$ a função que representa a taxa de $E x e c P A_{1}$ se, e somente se, ExecP $A_{1} \in E\left(M_{i}\right)$, temos que o rendimento de $\operatorname{ExecP} A_{1}$, denotado como $X\left(\operatorname{ExecP} A_{1}\right)$, será

$$
\begin{aligned}
X\left(E x e c P A_{1}\right) & =\sum_{M_{i} \in G} r\left(M_{i}\right) \pi_{i} \\
& =0 \times \pi_{1}+4 \times \pi_{2}+0 \times \pi_{3}+0 \times \pi_{4} \\
& =1.32626
\end{aligned}
$$

Com base no grafo de alcançabilidade e na distribuição estacionária da CMTC subjacente, é possível determinar ainda outras medidas de desempenho do sistema, definindo as funções de indexação correspondentes.

Tendo em vista que os elementos das RPEGs são mapeamentos de componentes de workflow, também é possível definir medidas de desempenho para esses componentes. No caso das atividades 
por exemplo, se consideramos as transições da RPEG geradas durante o mapeamento da atividade, é possível calcular seu rendimento, que é dado em função do seus requisitos de recursos. Além disso, podemos obter o número de instâncias executando a atividade, a quantidade em espera para ser executada, entre outros.

Tomando a atividade $A$ do workflow da Figura 3.2, pode-se ver que a transição ExecP $A_{1}$ representa a execução de $A$. Disso, podemos calcular a quantidade de trabalho por unidade de tempo feita na atividade $A$, como sendo: a quantidade de trabalho que realiza o recurso $R 1$ em $A$ (dos requisitos de $A$ ) pelo rendimento da transição $E x e c P A_{1}$, isto é $10 \times 1.32626=13.2626$ (quantidade de trabalho por unidade de tempo).

É importante destacar que as funções de indexação servem também na análise transiente, pois na análise transiente temos a distribuição dos estados para um determinado tempo. Além disso, neste tipo de análise podemos criar novas funções de indexação referentes ao sistema em tempo de execução, e.g., calcular o tempo em que um dado evento ocorre pela primeira vez.

A análise da evolução dos estados ao longo do tempo permite ter uma melhor visão de como o sistema está se comportando. No caso da RPEG da Figura 3.3, fazendo a análise transiente, podemos obter a evolução da distribuição das fichas nos lugares da respectiva RPEG, como mostrado na Figura 3.11. Em uma configuração com 1 instância de workflow $(I=1)$ e duas unidades do recurso $\left(N R_{1}=2\right)$, no estado estacionário temos que: $\mathbb{E}\left(P_{0}\right)=0.00265252, \mathbb{E}\left(P_{1}\right)=0.331565, \mathbb{E}\left(P_{2}\right)=$ $0.00265252, \mathbb{E}\left(P_{3}\right)=0.66313$ e $\mathbb{E}\left(R_{1}\right)=1.00531$. Isto implica que temos uma unidade de recurso que não está sendo usada, como indicado pela trajetória de $R_{1}$.

Se mudamos a configuração inicial para a RPEG da Figura 3.3, fazendo $I=3$, temos que as trajetórias que descrevem a distribuição de fichas nos lugares da rede evoluem como na Figura 3.12. No estado estacionário, temos que: $\mathbb{E}\left(P_{0}\right)=0.50402, \mathbb{E}\left(P_{1}\right)=0.664011, \mathbb{E}\left(P_{2}\right)=0.503947, \mathbb{E}\left(P_{3}\right)=$ 1.32802 e $\mathbb{E}\left(R_{1}\right)=0.00796754$. Aqui podemos ver que aproximadamente duas instâncias estão sendo executadas $\left(\mathbb{E}\left(P_{1}\right)+\mathbb{E}\left(P_{3}\right)=1.992031\right)$, então uma instância está esperando para ser executada $\left(\mathbb{E}\left(P_{0}\right)+\mathbb{E}\left(P_{2}\right)=1.007967\right)$, isto devido ao fato de termos apenas duas unidades de recurso disponíveis.

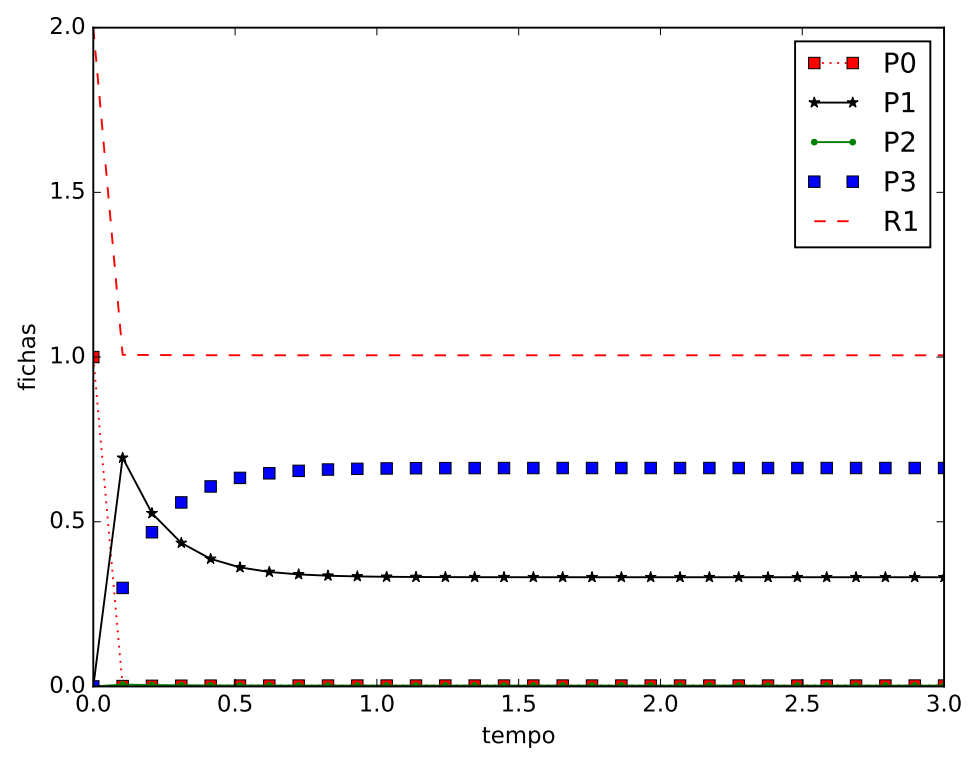

Figura 3.11: Evolução no tempo, das quantidades médias de fichas nos lugares da RPEG mostrada na Figura 3.3, calculados de acordo com o grafo de alcançabilidade da Figura 3.10.

Da mesma forma que fizemos a análise de desempenho para a RPEG da Figura 3.3, que repre- 


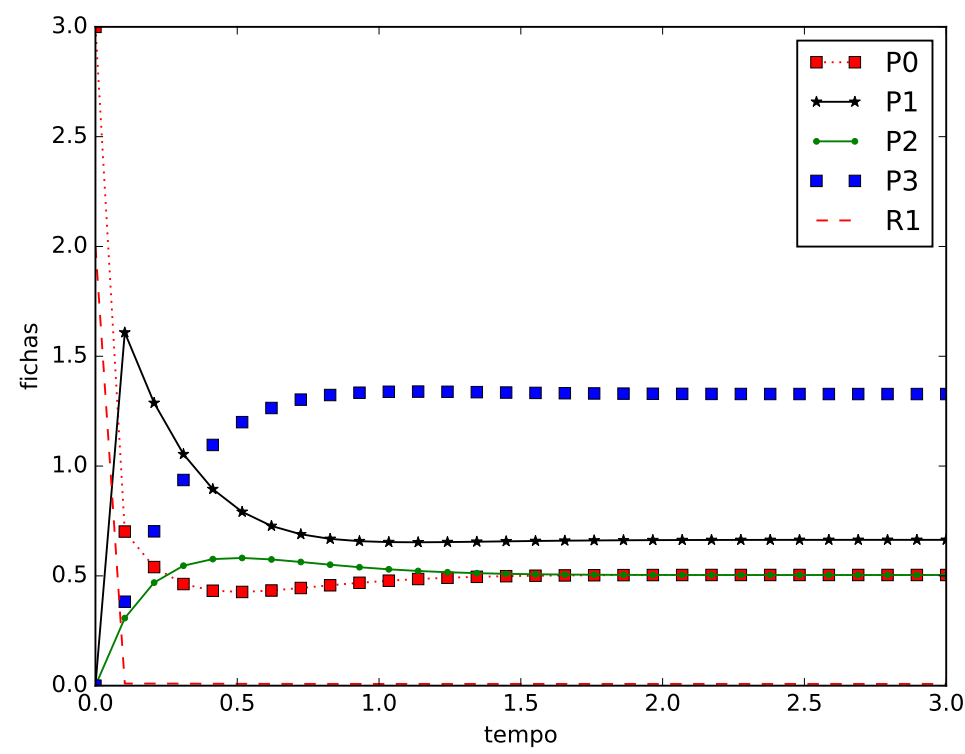

Figura 3.12: Trajetórias dos valores médios nos lugares na RPEG da Figura 3.3, quando mudamos o número de fichas inicial para $I=3$.

senta a estrutura de workflow sequencial da Figura 3.2, pode-se fazer a análise das outras estruturas de workflow. Supondo que temos as mesmas condições iniciais para as outras estruturas de workflow básicas $\left(A R_{1}=4, B R_{1}=40\right.$ e $\left.r=500\right)$, a seguir realizaremos a análise do desempenho desses modelos.

Para a estrutura de escolha (workflow da Figura 3.4), sejam $p r_{1}=0.8 \cdot r$ e $p r_{2}=0.2 \cdot r$. Temos as seguintes trajetórias do número médio de fichas por lugar, para diferentes números de fichas iniciais (valores de $I$ e $N R_{1}$ ):

- $I=5$ e $N R_{1}=10$; Figura 3.13, com estado estacionário: $\mathbb{E}\left(P_{0}\right)=0.0328947, \mathbb{E}\left(P_{1}\right)=$ $0.0263158, \mathbb{E}\left(P_{2}\right)=3.28947, \mathbb{E}\left(P_{3}\right)=0.00657894, \mathbb{E}\left(P_{4}\right)=1.64474$ e $\mathbb{E}\left(R_{1}\right)=5.06579$. Temos aproximadamente 5 instâncias sendo executadas $\left(\mathbb{E}\left(P_{2}\right)+\mathbb{E}\left(P_{4}\right)=4.93421\right)$, pois temos recursos suficientes.

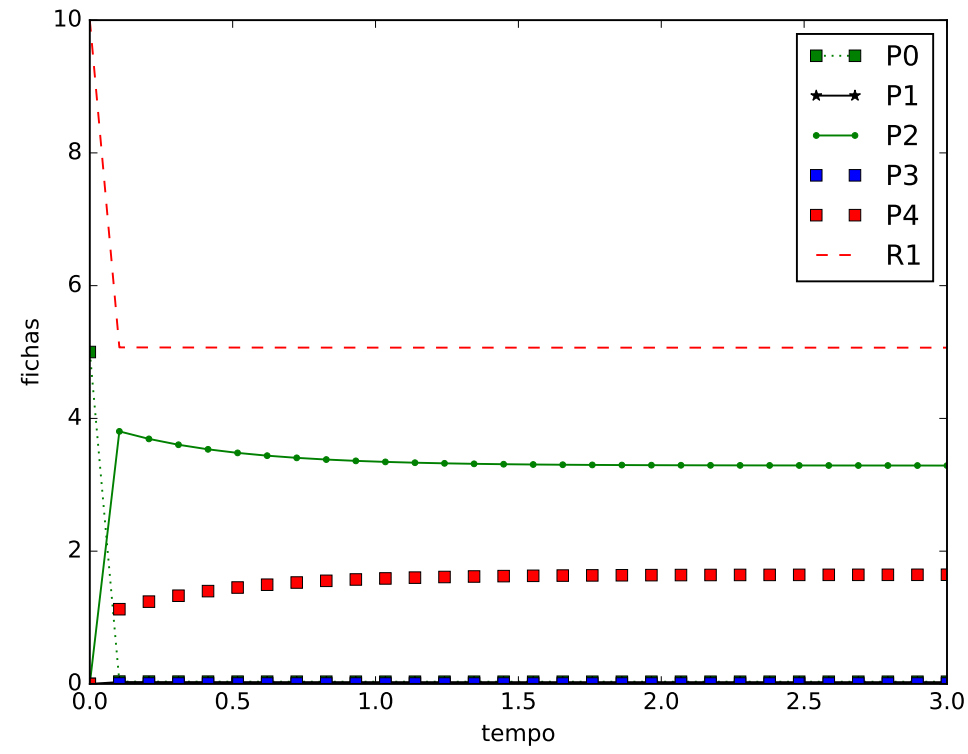

Figura 3.13: Trajetórias das quantidades médias de fichas nos lugares da RPEG da Figura 3.5, quando $I=5$ e $N R_{1}=10$. 
- $I=10$ e $N R_{1}=10$; Figura 3.14, com estado estacionário: $\mathbb{E}\left(P_{0}\right)=0.0657894, \mathbb{E}\left(P_{1}\right)=$ $0.0526315, \mathbb{E}\left(P_{2}\right)=6.57893, \mathbb{E}\left(P_{3}\right)=0.0131579, \mathbb{E}\left(P_{4}\right)=3.28949$ e $\mathbb{E}\left(R_{1}\right)=0.131579$. Agora são aproximadamente 10 instâncias sendo executadas $\left(\mathbb{E}\left(P_{2}\right)+\mathbb{E}\left(P_{4}\right)=9.86842\right)$ e, diferentemente do caso anterior, não temos recursos ociosos.

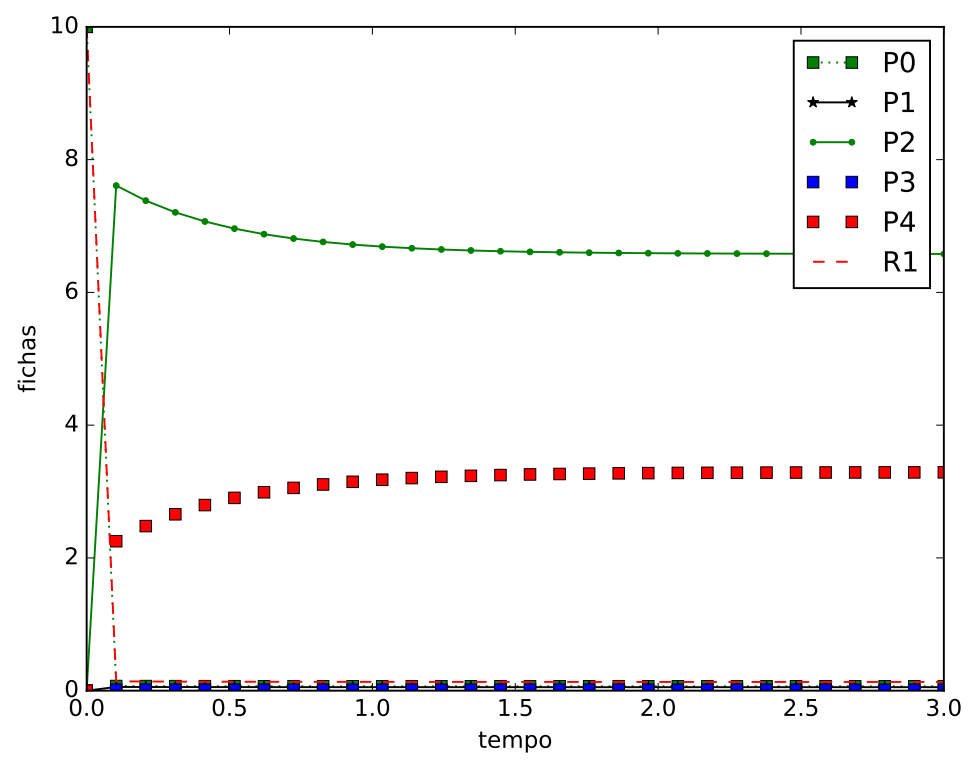

Figura 3.14: Trajetórias das quantidades médias de fichas nos lugares da RPEG da Figura 3.5, quando I = 10 e $N R_{1}=10$.

- $I=15$ e $N R_{1}=10$; Figura 3.15, com estado estacionário: $\mathbb{E}\left(P_{0}\right)=0.0663127, \mathbb{E}\left(P_{1}\right)=$ $4.54472, \mathbb{E}\left(P_{2}\right)=6.63126, \mathbb{E}\left(P_{3}\right)=0.442049, \mathbb{E}\left(P_{4}\right)=3.31566$ e $\mathbb{E}\left(R_{1}\right)=0.0530801$. Desta vez, já que temos mais instâncias que recursos, observamos que aumentou a quantidade de instâncias que esperam alocar recursos, são aproximadamente 5 delas $\left(\mathbb{E}\left(P_{0}\right)+\mathbb{E}\left(P_{1}\right)+\right.$ $\left.\mathbb{E}\left(P_{3}\right)=5.0530817\right)$.

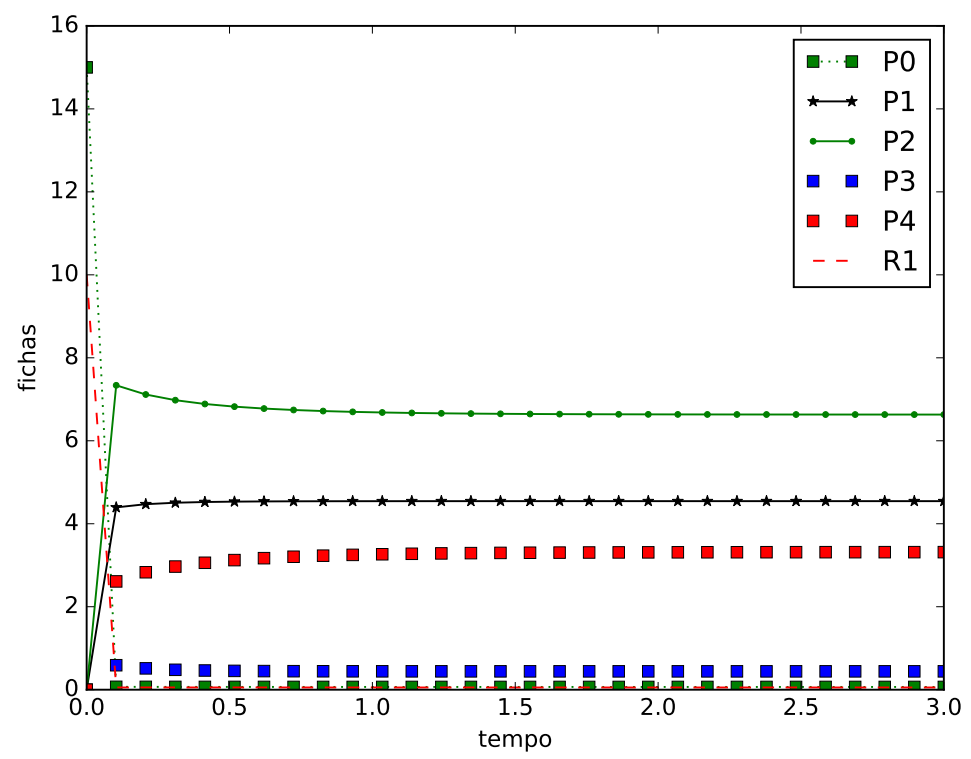

Figura 3.15: Trajetórias dos valores médios nos lugares da RPEG da Figura 3.5, quando $I=15$ e $N R_{1}=10$.

Agora, para a última estrutura básica, o paralelismo (workflow 3.4), temos as seguintes trajetórias do número médio de fichas por lugar, para diferentes números de fichas iniciais (valores de $I$ e $\left.N R_{1}\right)$ : 
- $I=5$ e $N R_{1}=10$; Figura 3.16, com estado estacionário: $\mathbb{E}\left(P_{0}\right)=0.00664011, \mathbb{E}\left(P_{1}\right)=$ $0.830013, \mathbb{E}\left(P_{2}\right)=0.00664011, \mathbb{E}\left(P_{3}\right)=1.66003, \mathbb{E}\left(P_{4}\right)=1.66002, \mathbb{E}\left(P_{5}\right)=0.0066401, \mathbb{E}\left(P_{6}\right)=$ 0.830011 e $\mathbb{E}\left(R_{1}\right)=5.01992$. Aqui temos aproximadamente 5 instâncias sendo executadas $\left(\mathbb{E}\left(P_{1}\right)+\mathbb{E}\left(P_{3}\right)+\mathbb{E}\left(P_{4}\right)+\mathbb{E}\left(P_{6}\right)=4.980074\right)$, portanto não temos instâncias esperando por recursos, contudo, são aproximadamente 5 unidades de recurso não utilizados.

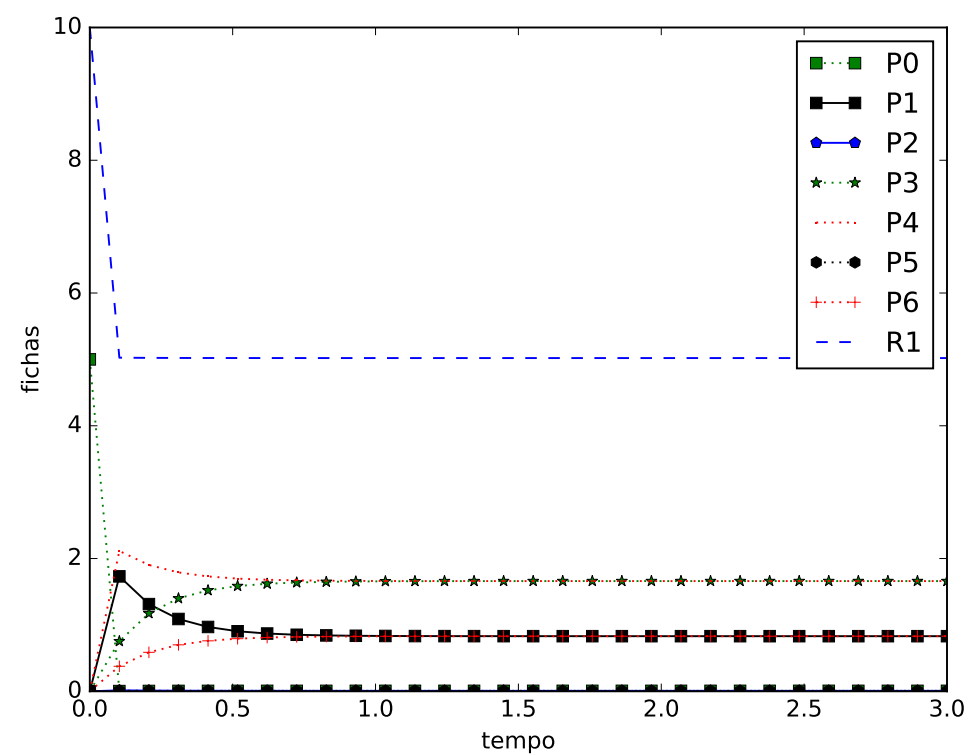

Figura 3.16: Trajetórias dos valores médios nos lugares da RPEG da Figura 3.7, quando $I=5$ e $N R_{1}=10$.

- $I=10$ e $N R_{1}=10$; Figura 3.17, com estado estacionário: $\mathbb{E}\left(P_{0}\right)=0.0132802, \mathbb{E}\left(P_{1}\right)=$ $1.66003, \mathbb{E}\left(P_{2}\right)=0.0132802, \mathbb{E}\left(P_{3}\right)=3.32009, \mathbb{E}\left(P_{4}\right)=3.32003, \mathbb{E}\left(P_{5}\right)=0.0132801, \mathbb{E}\left(P_{6}\right)=$ $1.66001 \mathrm{e} \mathbb{E}\left(R_{1}\right)=0.0398406$. Nesta configuração, aproximadamente todas as instâncias estão sendo executadas $\left(\mathbb{E}\left(P_{1}\right)+\mathbb{E}\left(P_{3}\right)+\mathbb{E}\left(P_{4}\right)+\mathbb{E}\left(P_{6}\right)=9.96016\right)$, utilizando todos os recursos disponíveis.

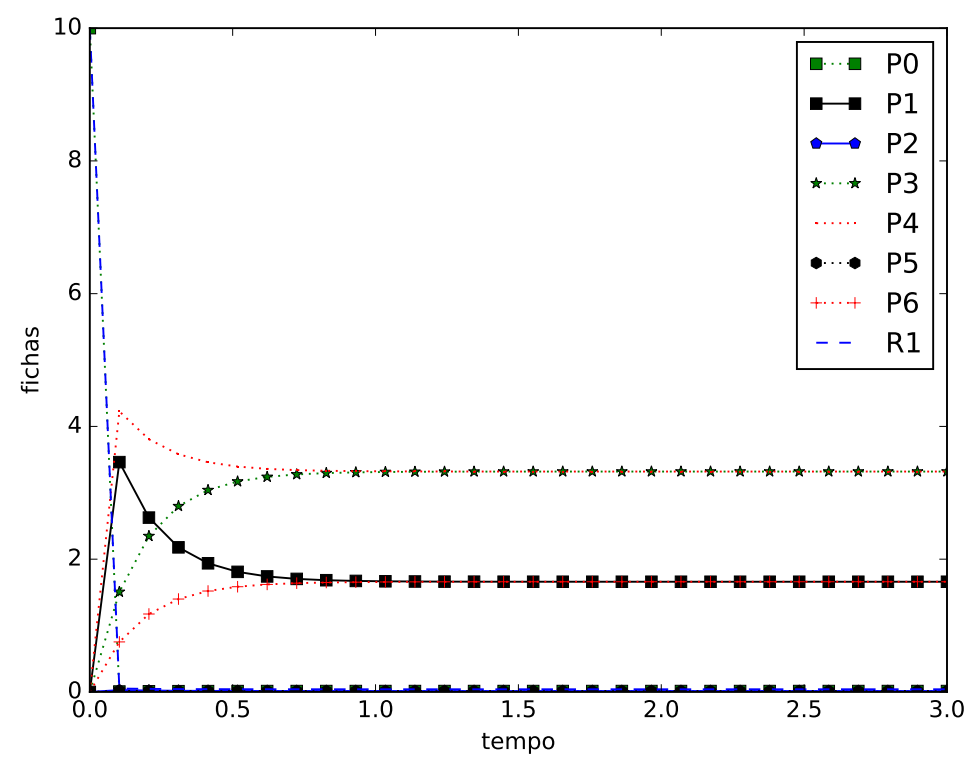

Figura 3.17: Trajetórias dos valores médios nos lugares da RPEG da Figura 3.7, quando $I=10$ e $N R_{1}=10$.

- $I=15$ e $N R_{1}=10$; Figura 3.18, com estado estacionário: $\mathbb{E}\left(P_{0}\right)=1.67235, \mathbb{E}\left(P_{1}\right)=$ $1.66367, \mathbb{E}\left(P_{2}\right)=1.67315, \mathbb{E}\left(P_{3}\right)=3.32803, \mathbb{E}\left(P_{4}\right)=3.32724, \mathbb{E}\left(P_{5}\right)=1.67225, \mathbb{E}\left(P_{6}\right)=$ 
1.66332 e $\mathbb{E}\left(R_{1}\right)=0.0177471$. Igualmente à configuração anterior, aproximadamente todos os recursos estão sendo utilizados $\left(\mathbb{E}\left(P_{1}\right)+\mathbb{E}\left(P_{3}\right)+\mathbb{E}\left(P_{4}\right)+\mathbb{E}\left(P_{6}\right)=9.98226\right)$, porém, temos aproximadamente 5 instâncias que não estão sendo executadas $\left(\mathbb{E}\left(P_{0}\right)+\mathbb{E}\left(P_{2}\right)+\mathbb{E}\left(P_{5}\right)=\right.$ 5.01775).

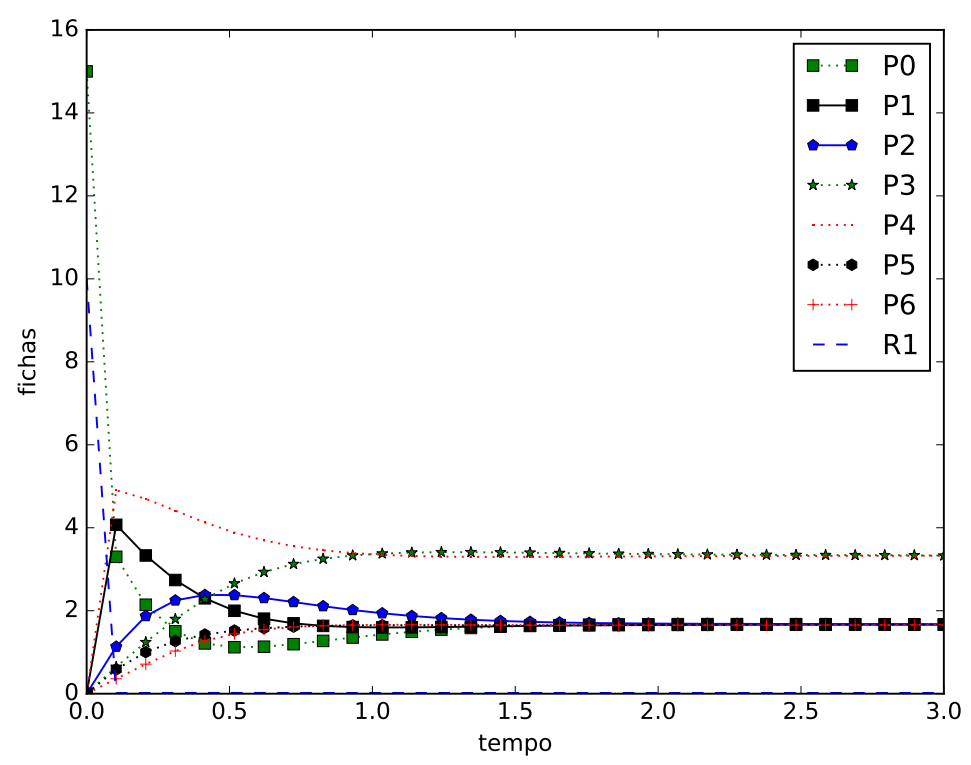

Figura 3.18: Trajetórias dos valores médios nos lugares da RPEG da Figura 3.7, quando $I=15$ e $N R_{1}=10$.

Então, utilizando as Redes de Petri é possível analisar o desempenho de sistemas no estado transiente ou no seu estado estacionário. Dessa forma é possível determinar medidas de desempenho para os workflows, utilizando modelagem analítica e especificando métricas (funções) de desempenho, de acordo com as especificações do sistema em questão.

\subsection{Explosão do Espaço de Estados}

Como visto anteriormente, os processos Markovianos permitem a modelagem de sistemas, assim como a análise de desempenho. Porém, essa técnica tem um baixo nível de abstração, o que dificulta o seu uso. Já as RPEGs, baseadas em processos de Markov, se mostraram um método mais conveniente para a modelagem analítica. Entretanto, sofrem do problema da explosão do espaço de estados. Para realizar a análise de uma RPEG é necessário obter o grafo de alcançabilidade, em outras palavras, todos os possíveis estados que pode atingir o sistema. O problema de alcançabilidade, como citado em [EN94, FK09], é um problema geralmente NP-completo, e polinomial para casos particulares.

Quando se usa processos Markovianos, temos uma visão microscópica do sistema. Isto significa que, em cada estado do sistema, é possível saber o estado de cada instância individualmente (nas RPEGs é como se colocássemos um rótulo em cada ficha). Dessa forma, o estado do sistema está representado pelo estado individual de cada instância, fazendo que o espaço de estados do sistema seja de ordem exponencial.

Uma propriedade das RPEGs utilizadas neste trabalho para o mapeamento dos workflows de negócio é a de que elas são $k$-delimitadas, onde $k$ é o máximo entre a quantidade de instâncias (fichas no lugar inicial) e a maior quantidade entre os recursos disponíveis. Este tipo de redes, como descrito na Seção 2.7, tem um espaço de estados não maior que $(k+1)^{N}$, onde $N$ é o número de lugares. Na Tabela 3.5 podemos observar o crescimento dos estados alcançáveis para a 
RPEG da Figura 3.3, que modela uma estrutura de sequência com apenas duas atividades. Para os experimentos, usamos um computador Intel(R) Core(TM) i5-3230M CPU @ 2.60GHz com 8GB de RAM.

\begin{tabular}{|c|c|c|c|c|c|}
\hline I (instâncias) & NR1 & Estados & Transições & Tempo (Seg.) & Memória (MB) \\
\hline 1 & 10 & 4 & 4 & 0.001 & 0.000104 \\
\hline 2 & 10 & 10 & 16 & 0.001 & 0.000213 \\
\hline 5 & 10 & 56 & 140 & 0.001 & 0.001082 \\
\hline 10 & 10 & 286 & 880 & 0.006 & 0.005691 \\
\hline 50 & 100 & 23426 & 88400 & 0.169 & 0.639781 \\
\hline 100 & 100 & 176851 & 686800 & 0.956 & 5.588984 \\
\hline 200 & 200 & 1373701 & 5413600 & 9.306 & 49.580363 \\
\hline 300 & 300 & 4590551 & 18180400 & 45.609 & 178.517314 \\
\hline 400 & 400 & 10827401 & 42987200 & 182.666 & 439.454154 \\
\hline 500 & 500 & 21084251 & 83834000 & 581.903 & 888.852269 \\
\hline
\end{tabular}

Tabela 3.5: Tamanho do grafo de alcançabilidade da RPEG da Figura 3.3 (em termos do número de estados e transições) e custo computacional (em termos de tempo de processamento e consumo de memória) para obtê-lo, para diferentes configurações iniciais no número de instâncias (I) e de recursos $\left(N R_{1}\right)$

Por outro lado, sabemos que o número de marcações alcançáveis representa o número de estados na CMTC subjacente ao sistema. Para fazer a análise de desempenho precisamos calcular as distribuições estacionária e transiente do processo. Calcular o estado estacionário tem complexidade $O\left(n^{3}\right)$ [BHHK03], ou $O\left(k n^{2}\right)$ utilizando métodos iterativos [Ste94] (e.g. POWER METHOD, GMRES, JOR, CGS), onde $n$ é o número de estados da cadeia e $k$ representa o número de iterações, que dependerá da estrutura da cadeia.

Além disso, o cálculo das medidas de desempenho, fazendo uso das Equações 2.3, 2.4 e 2.6, são de ordem $\Theta(n)$, ou seja, linear no número de estados, já que é necessário verificar cada um deles.

Dessa forma, para estruturas de workflow de negócio mais complexas, que ademais possuem muitas instâncias, a modelagem Markoviana é inviável pelo alto custo computacional em termos de tempo e memória. No próximo capítulo, exploramos o uso Teoria do Campo Médio, com a intenção de superar o problema da explosão do espaço de estados na modelagem analítica de worklows de negócio com numerosas instâncias. 


\section{Capítulo 4}

\section{Geração de espaços de estados contínuos para modelos de workflows}

De uma forma simples, a análise de processos estocásticos consiste em técnicas para modelar sistemas por meio de processos estocásticos e em métodos matemáticos para analisar os modelos em questão, geralmente técnicas de álgebra linear. A análise de processos estocásticos não é recente e tem evoluído ao longo do tempo. Porém, um problema muito recorrente nesse tipo de análise é o problema de explosão do espaço de estados, que faz com que as técnicas de análise não possam ser aplicadas sobre sistemas cujos modelos estão baseados em processos estocásticos.

Foi no contexto da busca por técnicas para análise de sistemas com grande número de estados que não fossem proibitivas (do ponto de vista computacional) que a Teoria do Campo Médio foi desenvolvida. Um outro fator que influenciou o seu desenvolvimento foi o cálculo estocástico [Kar91], que visa modelar processos estocásticos contínuos por meio do uso de equações diferenciais ordinárias (EDOs). As EDOs vêm sendo aplicadas como aproximações determinísticas de sistemas com comportamento estocástico.

Neste capítulo é apresentada a Teoria do Campo Médio, com o intuito de evitar o problema da explosão de estados. Abordaremos a geração de espaços de estados contínuos e também detalharemos a metodologia aplicada para derivar o modelo de Campo Médio a partir de modelos estocásticos. Essa metodologia baseia-se principalmente no trabalho de Bortolussi et al. [BHLM13].

\subsection{Teoria do Campo Médio (Mean Field Theory)}

Em muitas áreas (e.g., biologia, química, teoria de epidemias, física e finanças), as equações diferenciais estão sendo utilizadas para criar modelos determinísticos contínuos para processos dinâmicos próprios dessas áreas. Uma característica importante de tais modelos é que eles são discretos e aleatórios na sua evolução.

A Teoria do Campo Médio - TCM (do inglês Mean Field Theory) surgiu da estreita relação entre os campos da Física e da Estatística. No começo, para modelar sistemas dinâmicos, um dos métodos mais utilizados foram as cadeias de Markov. Depois, foram criados métodos de campo médio que geralmente têm como objetivo a resolução de problemas abordados com cadeias de Markov, mas por meio de técnicas matemáticas diferentes. A Teoria do Campo Médio é um método determinístico, enquanto as cadeias de Markov se baseiam em técnicas estocásticas. A Teoria do Campo Médio se relaciona com a Teoria de Otimização e a Teoria de Perturbações [OS01].

A TCM é amplamente usada na Física, para descrever a interação de sistemas multicorpos. A proposta é de trabalhar com o sistema multicorpos sem considerar explicitamente todas as interações das partículas entre dois corpos, mas descrevendo a interação de uma partícula com as outras considerando um potencial médio criado pelas outras partículas. 
O objetivo principal dessa teoria é aproximar as estatísticas de uma variável aleatória que esta correlacionada com outras variáveis aleatórias, supondo que é possível ter um "campo" médio com uma distribuição simples que representa a influência de todas as outras variáveis aleatórias [OW00].

Por meio da TCM podemos realizar aproximações determinísticas de sistemas estocásticos. Essas aproximações determinísticas são chamadas também de sistemas determinísticos. É importante destacar que os sistemas determinísticos podem ser de tempo discreto ou de tempo contínuo. Sistemas determinísticos de tempo discreto são aproximações de CMTDs síncronas ${ }^{1}$ [LMM07]; a partir de sua definição é que são desenvolvidas as aproximações determinísticas de tempo contínuo, para CMTCs e uma classe especial de CMTDs, as assíncronas ${ }^{2}$ [BHLM13]. Para este trabalho, é de especial interesse a aproximação determinística para CMTCs, que são os modelos subjacentes aos formalismos mais usados para a análise preditiva de desempenho de sistemas computacionais, que é o caso das RPEGs.

\subsubsection{Aproximação determinística de CMTDs síncronas}

A aproximação determinística de CMTDs síncronas é o tipo de aproximação determinística mais simples para processos de Markov [BHLM13], devido às condições do modelo. Além disso, sua formulação serve como base para a aproximação de modelos mais sofisticados.

Seja um sistema composto por $N$ objetos [LMM07, BGT08, GGL12, BCFH11, Hil14, BMP07, BLB08], chamados a partir de agora de entidades. Um conjunto $S$ de estados, com $|S|=n$, representa os possíveis estados para cada entidade do sistema. O estado da entidade $i$ no tempo $t \in \mathbb{N}$ (o tempo é discreto) é representado por $Y_{i}^{N}(t)$. Logo, o estado do sistema no tempo $t$ é dado por:

$$
Y^{(N)}(t)=\left\{Y_{i}^{(N)}(t) ; 1 \leqslant i \leqslant N\right\}
$$

que tem como espaço de estados $S^{N}$ (cuja cardinalidade é $n^{N}$ ).

Para poder reduzir o espaço de estados do sistema, temos como condição que todas as suas entidades sejam idênticas e indistinguíveis. Dessa forma, o comportamento de uma entidade $i$ não depende do estado de alguma outra entidade específica, mas sim do número de entidades que existem em cada estado. Portanto, o estado do sistema todo no tempo $t$ pode ser descrito e aproximado pelo seu processo de contagem $X^{(N)}(t)=\left\{X_{i}^{(N)}(t) ; i \in S\right\}$, onde:

$$
\begin{aligned}
& X_{i}^{(N)}(t)=\sum_{j=1}^{N} \mathbf{1}\left\{Y_{j}^{(N)}(t)=i\right\} \\
& \mathbf{1}\left\{Y_{j}^{(N)}(t)=i\right\}= \begin{cases}1 & \text { se } Y_{j}^{(N)}(t)=i \\
0 & \text { c.c. }\end{cases}
\end{aligned}
$$

ou seja, $X_{i}^{(N)}(t) \in\{0, \ldots, N\}$ representa o número de entidades no estado $i$ no tempo $t$. Além disso, deve-se verificar que $\sum_{i \in S} X_{i}^{(N)}(t)=N, \forall t \geqslant 0$. A configuração inicial do sistema, estado inicial, é $X^{(N)}(0)=d_{0}^{(N)}$.

\footnotetext{
${ }^{1}$ Uma CMTD síncrona é aquela que descreve um conjunto de agentes que evoluem de forma sincronizada em cada passo (intervalo de tempo discreto).

${ }^{2}$ Nas CMTDs assíncronas, é escolhido um número constante de agentes que evoluem de forma sincronizada em cada unidade de tempo discreto, diferentemente das CMTDs síncronas.
} 
Agora, o espaço de estados não tem mais tamanho $n^{N}$, já que foi reduzido para $\left(\begin{array}{c}N+n-1 \\ n-1\end{array}\right)$.

A partir de $X^{(N)}(t)$ podemos definir as variáveis de contagem normalizadas, também chamadas de medidas de ocupação:

$$
\hat{X}^{(N)}(t)=\left\{\frac{1}{N} X_{i}^{(N)}(t) ; i \in S\right\}
$$

onde cada medida $\hat{X}_{i}^{(N)}(t)$ representa a proporção de entidades que estão no estado $i$ no tempo $t$, podendo ser interpretada também como a probabilidade de uma entidade estar no estado $i$ no tempo $t$. Além disso, pode-se ver que $\sum_{i \in S} \hat{X}_{i}^{(N)}(t)=1, \forall t \geqslant 0$, e a configuração inicial será $\hat{d}_{0}^{(N)}=d_{0}^{(N)} / N$. O vetor $\hat{X}^{(N)}(t)$ contém as variáveis de contagem normalizadas e tem valores no simplex unitário $v^{n}$. Este vetor descreve o estado global do sistema.

Por meio das variáveis de contagem normalizadas, podemos definir uma matriz de probabilidades, que mostra a probabilidade de uma entidade mudar de estado. Seja $P^{(N)}(\hat{d})$ a matriz $(n \times n)$ de probabilidades para $\hat{d} \in v^{n}$, definida pela função $P^{(N)}: v^{n} \rightarrow[0,1]^{n^{2}}$. Seja uma entidade qualquer $k$, a probabilidade de $k$, cujo estado é $i$ no tempo $t$, mudar para o estado $j$, dado que $\hat{X}^{(N)}(t)=\hat{d}$, é definida como:

$$
P_{i j}^{(N)}(\hat{d})=\mathbb{P}\left(Y_{k}^{(N)}(t+1)=j \mid Y_{k}^{(N)}(t)=i, \hat{X}^{(N)}(t)=\hat{d}\right)
$$

Logo, podemos definir uma CMTD que descreve a evolução das variáveis de contagem $X^{(N)}$, por meio da matriz de probabilidades definida por $P^{(N)}$. Para definir a evolução, para cada tempo $t$ são introduzidos vetores aleatórios $B_{i}\left(X_{i}^{(N)}(t), P_{i}^{(N)}\left(\hat{X}^{(N)}(t)\right)\right)$, com $i \in S$, dependentes do estado atual $X^{(N)}(t)$ e que seguem uma distribuição binomial ${ }^{4}$, onde $P_{i}^{(N)}\left(\hat{X}^{(N)}(t)\right)$ é a i-ésima fila da matriz $P^{(N)}\left(\hat{X}^{(N)}(t)\right)$. Seja $B_{i j}$ o j-ésimo elemento do vetor $B_{i}$, temos que

$$
X_{j}^{(N)}(t+1)=\sum_{i=1}^{n} B_{i j}\left(X_{i}^{(N)}(t), P_{i}^{(N)}\left(\hat{X}^{(N)}(t)\right)\right)
$$

A partir da Equação 4.1 podemos definir a equação para as variáveis de contagem normalizadas:

$$
\hat{X}_{j}^{(N)}(t+1)=\sum_{i=1}^{n} \frac{1}{N} B_{i j}\left(N \hat{X}_{i}^{(N)}(t), P_{i}^{(N)}\left(\hat{X}^{(N)}(t)\right)\right)
$$

\subsubsection{Sistema Determinístico de Tempo Discreto}

Um Sistema Determinístico de Tempo Discreto (SDTD) está em tempo discreto mas tem o espaço de estados contínuo, aliviando assim o problema de explosão de estados. Formalmente, um SDTD $\hat{x}(t)$, em $E \subseteq \mathbb{R}^{n}$ é dado pela função $f: E \rightarrow E$ e um ponto inicial $\hat{x}(0)=\hat{d}_{0}$. A relação $\hat{x}(t+1)=f(\hat{x}(t))$ é válida, gerando equações de diferença.

Para calcular a aproximação determinística para as variáveis de contagem normalizadas, primeiro supomos que existe uma função contínua $P(\hat{d})$, independente de $N$, tal que $P^{(N)}(\hat{d})$ converge

\footnotetext{
${ }^{3}$ O simplex unitário $v^{n}$ é o conjunto de pontos em $\mathbb{R}^{n}$, cujas coordenadas são não negativas e a soma delas é igual a 1, ou seja, $v^{n}=\left\{\hat{d} \in[0,1]^{n} \mid \sum_{i=1}^{n} \hat{d}_{i}=1\right\}$.

${ }^{4} \mathrm{~A}$ distribuição binomial, com parâmetros $(n, p)$, é uma distribuição probabilística discreta que representa o número de sucessos em $n$ tentativas independentes, com probabilidade $p$ de sucesso [Ros14].
} 
uniformemente para $P(\hat{d})$, isto é $\lim _{N \rightarrow \infty} \sup _{\hat{d} \in v^{n}}\left\|P^{(N)}(\hat{d})-P(\hat{d})\right\|=0$.

A Equação 4.2 pode ser reescrita como

$$
\hat{X}_{j}^{(N)}(t+1)=\sum_{i=1}^{n} \hat{X}_{i}^{(N)}(t) \frac{1}{N \hat{X}_{i}^{(N)}(t)} B_{i j}\left(N \hat{X}_{i}^{(N)}(t), P_{i}^{(N)}\left(\hat{X}^{(N)}(t)\right)\right)
$$

e para $\hat{X}_{i}^{(N)}(t)=0$, o somando será zero.

Quando $N$ vai para infinito, vamos supor que $\hat{X}^{(N)}(t)$ converge quase certamente para o valor $\hat{x}(t)$, ou seja seus comportamentos serão parecidos. Logo, na Equação 4.3 é possível aplicar a lei dos grandes números [Ros14] (para a distribuição binomial, quase certamente $\frac{1}{N} B(N, p) \rightarrow p$ quando $N \rightarrow \infty$ ), tendo que quase certamente:

$$
\lim _{N \rightarrow \infty} \hat{X}_{j}^{(N)}(t+1)=\sum_{i=1}^{n} \hat{x}_{i}(t) P_{i j}(\hat{x}(t)) .
$$

Portanto, se definirmos o seguinte SDTD:

$$
\left\{\begin{array}{l}
\hat{x}(t+1)=\hat{x}(t) \cdot P(\hat{x}(t)) \\
\hat{x}(0)=\hat{d}_{0}
\end{array}\right.
$$

e supormos que $\hat{d}_{0}^{(N)}$ converge quase certamente para $\hat{d}_{0}$, aplicando a lei dos grandes números no tempo $t$ é possível provar o seguinte teorema:

Teorema 4.1.1. (Aproximação de Campo Médio em Tempo Discreto [LMMO7])

Seja $\hat{x}(t)$ o SDTD definido em 4.4. Se supomos que $\hat{d}_{0}^{(N)} \rightarrow \hat{d}_{0}$ quase certamente, deduzimos que $\hat{X}_{0}^{(N)}=\hat{x}(0)$ (medida de ocupação inicial). Da mesma forma, vamos supor que $P^{(N)}(\hat{d})$ converge uniformemente para a função contínua $P(\hat{d})$. Então, para $\hat{X}^{(N)}(t)$, onde $t>0$, temos que:

$$
\lim _{N \rightarrow \infty} \hat{X}^{(N)}(t)=\hat{x}(t)
$$

isto é, $\hat{x}(t)$ é a aproximação determinística da medida de ocupação para $N \rightarrow \infty$.

\subsubsection{Aproximação determinística de CMTCs}

Como mencionado anteriormente, usaremos esta aproximação para a análise de desempenho de workflows bem-definidos, conforme especificado na Seção 2.2, já que existe uma CTMC subjacente a este tipo de workflows.

CTMCs são denominadas assíncronas, pois diferentemente das CMTDs síncronas, nelas não existem dois eventos em um mesmo instante de tempo, devido à continuidade da distribuição exponencial. Contudo, neste tipo de sistema, quanto mais entidades o sistema possui, maior o número de eventos por unidade de tempo. Assim, supondo infinitas entidades, a densidade dos eventos será infinita, e um único evento causará um impacto infinitesimal nas variáveis de contagem.

Para formalizar este tipo de sistema, faremos uso do modelo apresentado por Bortolussi et al. [BHLM13] para representar CMTCs de populações, que, diferentemente da descrição tradicional de processos de Markov, especificam as transições parametricamente. A Definição 4.1.1 introduz estes modelos.

Definição 4.1.1. Uma CMTC de população - CMTCP é uma tupla $\chi_{C}=\left(X, D, T, d_{0}\right)$, onde: 
- $X=\left(X_{1}, \ldots, X_{n}\right)$ é o conjunto das variáveis de contagem.

- Uma variável $X_{i} \in X$ toma valores no conjunto $D_{i} \subset \mathbb{R}$, assim, o espaço de estados do modelo está dado por $D=\prod_{i} D_{i}$.

- $d_{0} \in D$ é o estado inicial (configuração inicial).

- $T=\left\{\tau_{1}, \ldots, \tau_{m}\right\}$ é conjunto de transições $\tau_{i}=(a, s, t, r)$, onde:

- a é o nome da transição,

- $s \in \mathbb{R}^{n}, s \geqslant 0$, (pré-vetor) indica o número de instâncias de cada variável consumidas por $\tau_{i}$.

- $t \in \mathbb{R}^{n}, t \geqslant 0$, (pós-vetor) oposto a s, especifica quantas instâncias são criadas por $\tau_{i}$ em cada variável. Além disso, podemos definir $v=t-s$ que mede a mudança no estado do modelo causada por $\tau_{i}$; v é chamado de vetor mudança de estado.

- $r: D \rightarrow \mathbb{R}_{\geq 0}$ representa a taxa de $\tau_{i}$. Para $d \in D$, se $d+v \notin D$, vale que $r(d)=0$.

Para $\chi^{(N)}$, que é uma CMTCP dependente da população total $N$, podemos definir a CMTC subjacente, cujo espaço de estados é $D^{(N)}$, e sua matriz infinitesimal é dada por:

$$
Q\left(d, d^{\prime}\right)= \begin{cases}\sum_{\tau \in T^{(N)} \mid v_{\tau}=d^{\prime}-d} r_{\tau}^{(N)}(d), & \text { se } d \neq d^{\prime} \\ -\sum_{d^{\prime \prime} \neq d} Q\left(d, d^{\prime \prime}\right), & \text { c.c. }\end{cases}
$$

Para expressar a dependência da CMTCP $\chi^{(N)}$ na população total $N$, é necessário derivar um modelo normalizado, análogo à medida de ocupação para o modelo discreto. Já que o limite determinístico se aproxima do sistema inicial quando $N$ aumenta, definimos $\gamma_{N}$ como sendo o tamanho do sistema com $N$ entidades. Além disso, este valor deve permanecer constante ao longo do tempo. Já que a normalização consiste em dividir as variáveis pelo tamanho do sistema, deve verificar-se que $\lim _{N \rightarrow \infty} \gamma_{N}=\infty$, dessa forma as variáveis normalizadas serão contínuas. Uma configuração comumente usada é $\gamma_{N}=N$, a qual também será usada neste trabalho. Seja $\delta_{N}=1 / \gamma_{N}$ o incremento de intervalo básico, ou seja, o sistema evolui em intervalos de tamanho $\delta_{N}$, onde $\lim _{N \rightarrow \infty} \delta_{N}=0$.

No modelo de CMTCP, a normalização afeta não apenas as variáveis, mas também todos os parâmetros da Definição 4.1.1. Assim, para a CMTCP $\chi^{(N)}=\left(X^{(N)}, D^{(N)}, T^{(N)}, d_{0}^{(N)}\right)$, o modelo normalizado correspondente é $\hat{\chi}^{(N)}=\left(\hat{X}^{(N)}, \hat{D}^{(N)}, \hat{T}^{(N)}, \hat{d}_{0}^{(N)}\right)$, onde:

- $\hat{X}^{(N)}=\delta_{N} \cdot X^{(N)}$,

- $\hat{D}^{(N)}=\left\{\hat{d} \mid \hat{d}=\delta_{N} \cdot d, d \in D^{(N)}\right\}$,

- $\forall \tau \in T^{(N)}$, sendo que $\tau=\left(a, s^{(N)}, t^{(N)}, r^{(N)}\right)$, temos $\hat{\tau}=\left(a, \hat{s}^{(N)}, \hat{t}^{(N)}, \hat{r}^{(N)}\right)$, com:

- $\hat{s}^{(N)}=\delta_{N} \cdot s^{(N)}$,

- $\hat{t}^{(N)}=\delta_{N} \cdot t^{(N)}$, portanto $\hat{v}^{(N)}=\delta_{N} \cdot v^{(N)}$,

- $\hat{r}^{(N)}(\hat{d})=r^{(N)}\left(\gamma_{N} \cdot \hat{d}\right)$, para todo $\hat{d} \in \hat{D}$

- $\hat{T}^{(N)}=\left\{\hat{\tau} \mid \tau \in T^{(N)}\right\}$ 


\subsubsection{Sistema Determinístico de Tempo Contínuo}

Para que uma CMTC possa ser aproximada deterministicamente é preciso que, em conjunto, tanto a magnitude da mudança de estado para cada transição $\left(\left\|v^{(N)}\right\|\right)$ quanto o tempo entre eventos consecutivos sejam zero [Kur70, LMM07, BHLM13]. Dessa forma, podemos aproximar a magnitude e o tempo por variáveis contínuas para obter um sistema determinístico de tempo contínuo - SDTC. A seguir, são definidas as condições sob as quais existe a aproximação determinística.

Em primeiro lugar, seja $\hat{\chi}^{(N)}=\left(\hat{X}^{(N)}, \hat{D}^{(N)}, \hat{T}^{(N)}, \hat{d}_{0}^{(N)}\right)$ uma CMTCP normalizada. Definiremos as seguintes medidas de interesse:

- Taxa de saída - definida pela função $R^{(N)}: \hat{D}^{(N)} \rightarrow \mathbb{R}$, que representa a velocidade em que acontece uma transição para o estado $\hat{d}$, logo:

$$
R^{(N)}(\hat{d})=\sum_{\tau \in \hat{T}^{(N)}} \hat{r}_{\tau}^{(N)}(\hat{d})
$$

- Incremento médio - dado pela função $\mu^{(N)}: \hat{D}^{(N)} \rightarrow \mathbb{R}^{n}$, que define a variação média de cada variável em um intervalo de tempo, dado por:

$$
\mu^{(N)}(\hat{d})=\sum_{\tau \in \hat{T}^{(N)}} \hat{v}_{\tau}^{(N)} \frac{\hat{r}_{\tau}^{(N)}(\hat{d})}{R^{(N)}(\hat{d})}
$$

onde $\frac{\hat{r}_{\tau}^{(N)}(\hat{d})}{R^{(N)}(\hat{d})}$ é a probabilidade da transição $\tau$ acontecer.

- Matriz de covariância - é a matriz $\Sigma^{(N)}(\hat{d})$, que contêm as covariâncias dos incrementos entre cada par de variáveis, definida como:

$$
\Sigma_{i j}^{(N)}(\hat{d})=\sum_{\tau \in \hat{T}^{(N)}} \hat{v}_{\tau, i}^{(N)} \hat{v}_{\tau, j}^{(N)} \frac{\hat{r}_{\tau}^{(N)}(\hat{d})}{R^{(N)}(\hat{d})}-\mu_{i}^{(N)}(\hat{d}) \mu_{j}^{(N)}(\hat{d})
$$

- Dinâmica média ou deriva (drift) - é a função $F^{(N)}: \hat{D}^{(N)} \rightarrow \mathbb{R}^{n}$, que descreve a variação local da CMTC com o passar do tempo, está definida como:

$$
F^{(N)}(\hat{d})=R^{(N)}(\hat{d}) \mu^{(N)}(\hat{d})=\sum_{\tau \in \hat{T}^{(N)}} \hat{v}_{\tau}^{(N)} \hat{r}_{\tau}^{(N)}(\hat{d})
$$

Seja a sequência de processos $\left(\hat{\chi}^{(N)}\right)_{N \geqslant N_{0}}$ em relação a um sistema de tamanho crescente, onde $N_{0}$ é o tamanho inicial. Definimos um conjunto fechado $E$ em $\mathbb{R}^{n}$ tal que $\bigcup_{N} \hat{D}^{(N)} \subseteq E$, ou seja, $E$ engloba o espaço de estados da sequência de processos, e também da sua aproximação determinística. Contudo, a convergência é formulada sobre um subconjunto (apropriado) aberto $S \subseteq E$. A seguir, as condições de escalamento são definidas:

- Convergência da configuração inicial - supomos que existe $\hat{d}_{0} \in S$ tal que:

$$
\lim _{N \rightarrow \infty} \hat{d}_{0}^{(N)}=\hat{d}_{0}
$$


- Convergência da deriva - supomos que existe uma função Lipschitz $F: E \rightarrow \mathbb{R}^{n}$ tal que $F^{(N)}$ converge uniformemente para $F$, ou seja:

$$
\lim _{N \rightarrow \infty} \sup _{\hat{d} \in S^{(N)}}\left\|F^{(N)}(\hat{d})-F(\hat{d})\right\|=0
$$

- Convergência do ruído para zero - requer as seguintes condições:

- A taxa de saída seja delimitada para todo $N$, isto é, deve existir $\Lambda_{N} \in \mathbb{R}_{\geqslant 0}$ finito, tal que:

$$
\sup _{\hat{d} \in S^{(N)}} R^{(N)}(\hat{d})=\Lambda_{N}
$$

onde $S^{(N)}=S \cap \hat{D}^{(N)}$. Geralmente $\lim _{N \rightarrow \infty} \Lambda_{N}=\infty$, de modo que a frequência dos eventos aumenta com $N$.

- A magnitude das mudanças vá para zero, ou seja, deve existir $J_{N} \in \mathbb{R}_{\geqslant 0}$, de forma que:

$$
\max _{\tau \in \hat{T}^{(N)}}\left\|\hat{v}_{\tau}^{(N)}\right\|=J_{N}
$$

Além disso, $\lim _{N \rightarrow \infty} J_{N}=0$ e , mais especificamente, $J_{N}$ é $O\left(N^{-1}\right)$.

- O escalamento da taxa de saída e da magnitude das mudanças sejam compatíveis de acordo com o seguinte critério:

$$
J_{N}^{2} \Lambda_{N} \text { é } O\left(N_{-1}\right)
$$

indicando que a variação do sistema tende a zero.

As condições 4.6, 4.7, 4.8, 4.9 e 4.10 são conhecidas como condições de escalamento. Elas tornam-se mais simples de verificar quando os vetores de mudança de estados do modelo nãonormalizado são independentes de $N$, ou seja, para cada transição $\tau$ existe um vetor $v_{\tau}$ tal que $v_{\tau}^{(N)}=v_{\tau}$, assim $\hat{v}_{\tau}^{(N)}=\delta_{N} \cdot v_{\tau}$. Logo, temos que $J_{N}=\delta_{N} \max _{\tau \in T^{(N)}}\left\|v_{\tau}\right\|$, e já que $\left\|v_{\tau}\right\|$ é $O(1)$, temos que $J_{N}$ é $O\left(N^{-1}\right)$, então é verdadeiro que $J_{N}^{2} \Lambda_{N}$ é $O\left(N^{-1}\right)$.

Seja a CMTC $\hat{X}(t)$ subjacente à CMTCP $\hat{\chi}^{(N)}$. Além disso, seja $\hat{x}(t)$ a solução do problema de valor inicial (P.V.I.) $\frac{d \hat{x}(t)}{d t}=F(\hat{x}(t))$, com $\hat{x}(0)=\hat{d}_{0}$, ou seja, $\hat{x}(t)$ é a solução de equações diferenciais ordinárias (Ordinary Diferential Equations - ODEs). E, por último, seja $\zeta(S)=\inf \{t \geqslant$ $0 \mid \hat{x}(t) \notin S\}$ o tempo de saída de $\hat{x}(t)$ de $S$, e $\zeta^{(N)}(S)=\inf \left\{t \geqslant 0 \mid \hat{X}^{(N)}(t) \notin S\right\}$ o tempo de saída de $\hat{X}^{(N)}(t)$ de $S$. O seguinte teorema é válido:

Teorema 4.1.2. (Aproximação Determinística para CMTCPs)

Seja $\left(\hat{X}^{(N)}(t)\right)_{N \geqslant N_{0}}$ a sequência de CMTCs subjacente à sequência de CMTCPs $\left(\hat{\chi}^{(N)}\right)_{N \geqslant N_{0}}$ e $\hat{x}(t) a$ solução de ODEs (como descrito anteriormente). Além disso, suponha que as condições de escalamento são válidas. Para $\mathrm{T}<\zeta(S)$, temos que:

1. $\lim _{N \rightarrow \infty} \mathbb{P}(\zeta(S)<\mathrm{T})=0$,

2. $\forall \varepsilon \in \mathbb{R}_{>0}, \lim _{N \rightarrow \infty} \mathbb{P}\left(\sup _{0 \leqslant t \leqslant \mathrm{~T}}\left\|\hat{X}^{(N)}(t)-\hat{x}(t)\right\|>\varepsilon\right)=0$.

Além disso, $\mathbb{P}(\zeta(S)<\mathrm{T})$ e $\mathbb{P}\left(\sup _{0 \leqslant t \leqslant \mathrm{~T}}\left\|\hat{X}^{(N)}(t)-\hat{x}(t)\right\|>\varepsilon\right)$ são $O\left(e^{-N}\right)$. 
Este teorema estabelece a lei dos grandes números para CMTCPs, para um limite de tempo finito T tal que a solução das ODEs está em $S$ no intervalo $[0, T]$. Um resultado mais forte é estabelecido para $\zeta(S)<\infty$, sob a condição de $\hat{x}(t)$ sair de $S$ transversalmente no tempo $\zeta(S)$. Isto acontece se, e somente se, existe $\delta>0$ tal que, para $t \in] \zeta(S), \zeta(S)+\delta[\hat{x}(t) \notin c l(S)$, ou seja, $\hat{x}(t)$ permanece no fecho de $S$ por um tempo. Então temos:

Teorema 4.1.3. (Aproximação Determinística para CMTCPs com Tempos de Saída Finitos)

Seja $\left(\hat{X}^{(N)}(t)\right)_{N \geqslant N_{0}}$ a sequência de CMTCs subjacente à sequência de CMTCPs $\left(\hat{\chi}^{(N)}\right)_{N \geqslant N_{0}}$ e $\hat{x}(t) a$ solução de ODEs (como descrito anteriormente). Além disso, suponha que as condições de escalamento são válidas. Então, se $\zeta(S)<\infty$ e $\hat{x}(t)$ sai de $S$ transversalmente no tempo $\zeta(S)$, verifica-se para todo $\varepsilon \in \mathbb{R}_{>0}$ que:

1. $\lim _{N \rightarrow \infty} \mathbb{P}\left(\sup _{0 \leqslant t \leqslant \zeta(S)}\left\|\hat{X}^{(N)}\left(\min \left(t, \zeta^{(N)}(S)\right)\right)-\hat{x}(t)\right\|>\varepsilon\right)=0$,

2. $\lim _{N \rightarrow \infty} \mathbb{P}\left(\left\|\zeta^{(N)}(S)-\zeta(S)\right\|>\varepsilon\right)=0$.

O teorema estabelece que as trajetórias estocásticas e as determinísticas são idênticas, enquanto as ODEs permanecem em $S$. E também que os tempos de saída convergem; logo, pode-se aproximar $\zeta^{(N)}(S)$ por $\zeta(S)$.

Os teoremas 4.1.2 e 4.1.3 mostram que $\hat{X}^{(N)}(t)$ converge para $\hat{x}(t)$, ou seja, o modelo de CMTCPs pode ser aproximado por um SDTC expresso em forma de ODEs, que está em um espaço de estados contínuo, evitando o problema da explosão de espaço de estados.

\subsection{Aproximação Determinística para Workflows}

No Capítulo 3 mostramos como analisar quantitativamente workflows de negócio com grande quantidade de instâncias. Para esse fim, foram utilizadas as RPEGs, uma técnica baseada em processo de Markov. E, como foi indicado, existe uma dificuldade ao se utilizar métodos Markovianos - a explosão do espaço de estados - que para os modelos de workflow mostrados gera uma quantidade ingente de estados. Assim, impossibilita-se sua aplicação para modelos de maior escala (em estrutura e em número de instâncias).

Desse modo, é factível o uso da Teoria do Campo Médio para a modelagem e análise de workflows de negócio com numerosas instâncias. Na continuação, apresentamos o método desenvolvido neste trabalho para modelar workflows empregando a Teoria do Campo Médio, de tal forma que a aproximação esteja definida em um espaço de estados contínuo.

\subsubsection{Modelo de CMTCPs para Workflows}

Para definir a CMTCP que modela um workflow, seguiremos um processo análogo à modelagem de Redes de Petri descrito na Seção 3.1. Em primeiro lugar, é mandatório definir as variáveis de contagem, que representam os estados nos quais uma entidade pode estar, assim como as transições entre os estados. Para um workflow, podemos descrever os estados se considerarmos como é realizada a execução do sistema para uma única instância.

Com a finalidade de descrever os estados e as transições, analisaremos todos os componentes básicos dos workflows e, assim como nas RPEGs, mostraremos o mapeamento para cada um deles.

A descrição de mapeamento será definida de forma geral, porém, ao longo da descrição podemos mencionar como exemplo o workflow da Figura 4.1, denotado como $W f$, para o qual temos a seguinte descrição: 
- Recursos: $R_{1}=\left(R_{1}, 2,40\right)$ e $R_{2}=\left(R_{2}, 5,20\right)$

- Requisitos: $P_{A}=\left(\left[R_{1}, 10\right]\right), P_{B}=\left(\left[R_{1}, 20\right]\right)$ e $P_{C}=\left(\left[R_{1}, 5\right] \vee\left[R_{2}, 10\right]\right)$.

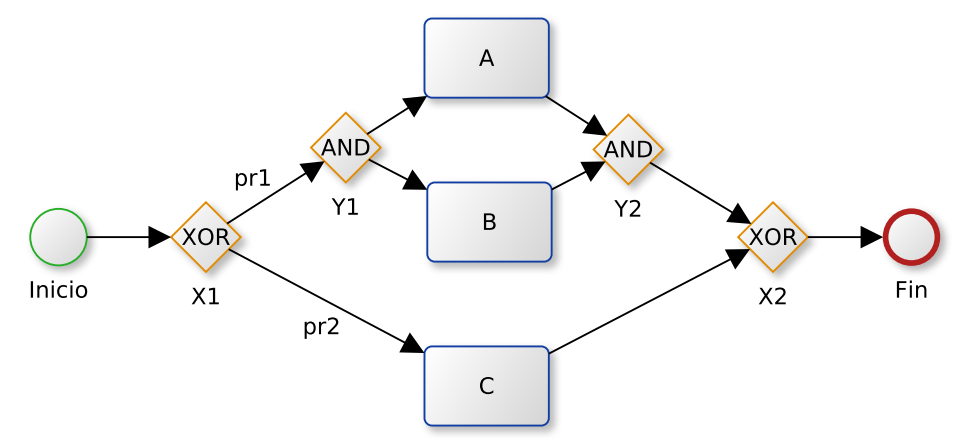

Figura 4.1: Modelo de workflow bem definido

Começamos considerando uma instância do workflow. Seja $S$ o conjunto de estados e $T$ o conjunto de transições da instância, tal que $\forall t_{i} \in T, t_{i}=(u, v, r)$, onde $u, v \in S$ e $r: S \times S \rightarrow \mathbb{R}_{\geqslant 0}$, ou seja, existe uma transição de $u$ para $v$ com taxa $r$. Além disso, seja $a \in S$ o estado inicial, e $z \in S$ o estado final (correspondentes aos eventos de início e fim do workflow respectivamente).

Na sequência, veremos a representação de atividade, escolha e paralelismo.

\section{Atividade}

Seja a atividade $A$ com requisitos $P_{A}=\left(P_{A 1} \vee \cdots P_{A x}\right)$, de acordo com a Definição 2.3.2. Também, seja $u \in S$ o estado da instância de workflow em que a atividade $A$ pode ser executada. Logo, já que para uma atividade ser executada primeiro é preciso alocar os recursos dos quais ela depende, o mapeamento da atividade segue duas fases: uma de alocação e outra de execução. Na fase de alocação de recursos temos as seguintes etapas:

(1) Para cada $P_{A i}$, com $1 \leqslant i \leqslant x$, criar um novo estado $s_{i}$ em $S$, que representa o fato da instância ter adquirido os recursos especificados em $P_{A i}$, sendo possível executar a atividade $A$.

(2) Criar uma nova transição de $u$ a cada $s_{i}$, para $1 \leqslant i \leqslant x$ (criados na etapa anterior), com taxa $A l c\left(P_{A i}\right)$ (a taxa de alocação de recursos). Seja $y$ o número de termos na conjunção $P_{A i}$, onde $P_{A i}=\left(\left[R_{i 1}, w_{1}\right] \wedge \cdots \wedge\left[R_{i y}, w_{y}\right]\right)$, temos que a taxa de alocação está definida como:

$$
\operatorname{Alc}\left(P_{A i}\right)=r \times \operatorname{Aval}\left(\left[R_{i 1}, w_{1}\right]\right) \times \ldots \times \operatorname{Aval}\left(\left[R_{i y}, w_{y}\right]\right)
$$

onde $\operatorname{Aval}(R)$ é a função que verifica a disponibilidade do recurso $R$; o valor dela está dado por:

$$
\operatorname{Aval}(R)=\operatorname{proporcao}(u) \times \min (\operatorname{esperando}(R), \operatorname{livres}(R))
$$

onde: $\operatorname{proporcao}(u)$ devolve a proporção de instâncias (em $u$ ) que precisam de $R$, dessa forma damos prioridade aos estados de acordo com o número de instâncias que estão esperando; esperando $(R)$ são o número total de instâncias esperando para alocar $R$ (disputa do recurso) e livres $(R)$ é a quantidade de recursos $R$ disponíveis. Assim garantimos que são alocados uma quantidade válida de recursos de acordo com a prioridade do estado $u$ (baseado na quantidade de instâncias nele). 
Supomos que os recursos são alocados paralelamente.

Depois, na fase da execução da atividade propriamente dita, seguimos os seguintes passos:

(1) Criar um novo estado $v$ em $S$; a instância de workflow estará nesse estado depois de ter executado a atividade $A$.

(2) Criar uma transição de cada $s_{i} \in S, 1 \leq i \leq x$, para o estado $v$ com taxa $\operatorname{Exec}\left(P_{A i}\right)$. Dado que $s_{i}$ representa a alocação dos recursos especificados em $P_{A i}$, a nova transição modela a execução de $A$, onde sua a taxa está definida como:

$$
\operatorname{Exec}\left(P_{A i}\right)=\min \left(\operatorname{Exec}\left(\left[R_{i 1}, w_{1}\right]\right), \ldots, \operatorname{Exec}\left(\left[R_{i y}, w_{y}\right]\right)\right)
$$

onde a taxa de execução de $A$ pelo recurso $R_{i j}$ está dada por:

$$
\operatorname{Exec}\left(\left[R_{i j}, w_{j}\right]\right)=\operatorname{Capacidade}\left(R_{i j}\right) / w_{j}
$$

sendo Capacidade $\left(R_{i j}\right)$ a capacidade de trabalho de $R_{i j}$ e $w_{j}$ a quantidade de trabalho a realizar em $A$ pelo recurso $R_{i j}$.

Portanto, $\operatorname{Exec}\left(P_{A i}\right)$ considera o tempo de execução do recurso em $P_{A i}$ que demora mais para executar a atividade $A$, visto que a taxa é o inverso do tempo de execução.

Graficamente, as fases de alocação de recursos e execução para uma atividade podem ser visualizadas na Figura 4.2.

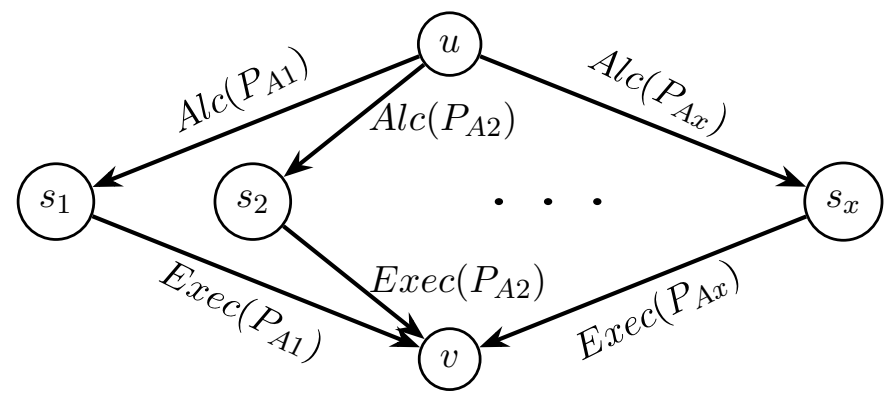

Figura 4.2: Estados e transições gerados no mapeamento da atividade A.

\section{Paralelismo}

Uma estrutura de paralelismo é definida por dois componentes, o $A N D$-split e o $A N D$-join. Então, precisamos descrever a representação de todos os fluxos paralelos compreendidos entre esses dois componentes. Sejam, $Y_{1}$ o componente $A N D$-split e $Y_{2}$ o $A N D$-join, e $\left\{F_{1}, \cdots, F_{x}\right\}$ a sequência de fluxos paralelos (que saem de $Y_{1}$ ). Além disso, suponhamos que $u \in S$ é o estado no qual a instância de workflow pode começar a executar $Y_{1}$; logo, o mapeamento do paralelismo é feito como segue:

(1) Suponha que $B_{i}=\left\{S\left(B_{i}\right), T\left(B_{i}\right)\right\}, S\left(B_{i}\right) \subset S$ e $T\left(B_{i}\right) \subset T$ representa o conjunto de estados $\left(S\left(B_{i}\right)\right)$ e transições $\left(T\left(B_{i}\right)\right)$ do mapeamento de $F_{i}$, com $1 \leq i \leq x$, tal que $B_{i}$ tem apenas um estado inicial denotado por $a\left(B_{i}\right) \in S\left(B_{i}\right)$ e também só um estado final $z\left(B_{i}\right) \in S\left(B_{i}\right)$. Seja $B=\left\{B_{i} \mid 1 \leq i \leq x\right\}$.

(2) Criar um novo estado $v$ em $S$, que representa o fim da execução do paralelismo; 
(3) Para cada possível permutação $\sigma=\left\{\sigma\left(B_{1}\right), \ldots, \sigma\left(B_{x}\right)\right\}$ de $B$, mapeamos $\sigma$ da seguinte forma:

- Juntar os estados $a\left(\sigma\left(B_{1}\right)\right)$ e $u$, i.e., fazer $a\left(\sigma\left(B_{1}\right)\right)=u$; dessa forma todas as permutações começarão em $u$;

- Juntar os estados $a\left(\sigma\left(B_{i+1}\right)\right)$ e $z\left(\sigma\left(B_{i}\right)\right)$, i.e., fazer $a\left(\sigma\left(B_{i+1}\right)\right)=z\left(\sigma\left(B_{i}\right)\right)$, for $1 \leq i<x-1$; ou seja, fazer que o estado inicial de cada componente seja o estado final do componente imediatamente anterior;

- Por último, criar uma transição de $z\left(\sigma\left(B_{x}\right)\right)$ para $v$, esta transição será rotulada como null. Essas transições são apenas necessárias na etapa de mapeamento, porém não farão parte do modelo final.

A representação gráfica de uma permutação de $B$ é mostrada na Figura 4.3, onde $u$ representa o começo do paralelismo, isto é, o componente $Y_{1}$, e o estado $v$ representa o final do paralelismo, indicado pelo componente $Y_{2}$.

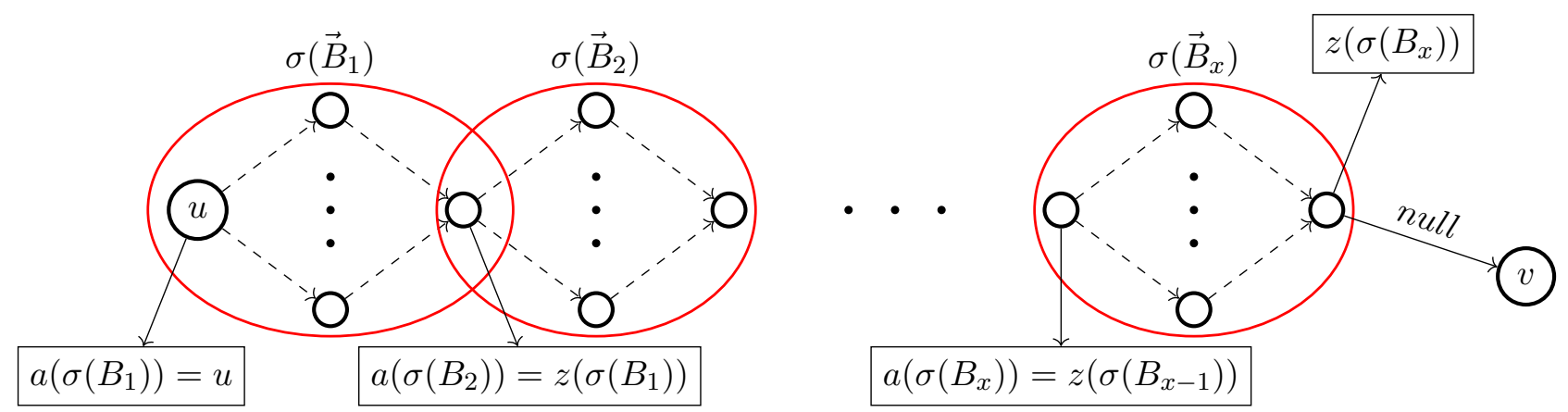

Figura 4.3: Representação de estados e transições da permutação $\sigma=\left(\sigma\left(B_{1}\right), \ldots, \sigma\left(B_{x}\right)\right)$ (para o mapeamento do paralelismo).

É importante ressaltar que este tipo de modelagem funcionará pelo fato das permutações obtidas serem equivalentes, sob a perspetiva quantitativa. As variáveis aleatórias utilizadas seguem uma distribuição exponencial, assim, fazendo uso da propriedade de perda de memória dessa distribuição pode-se demostrar a equivalência quantitativa das permutações.

Teorema 4.2.1. Sejam as atividades $A$ e $B$, cujos tempos de execução são descritos pelas variáveis aleatórias $X$ e $Y$ respetivamente. As duas variáveis seguem a distribuição exponencial com taxas $\alpha$ e $\beta$ respetivamente. Então, pela propriedade de perda de memória da distribuição exponencial, o tempo de execução dessas atividades é independente da ordem em que são executadas.

A propriedade de perda de memória da distribuição exponencial diz que para cada tempo em que uma atividade começa mas ainda não acabou, o tempo restante da atividade é distribuída da mesma forma como a duração da atividade completa.

\section{Escolha}

Os componentes que são usados numa estrutura de escolha são o XOR-split e o XOR-join. Então, para o mapeamento da escolha, procedemos da seguinte maneira:

- Se precisamos mapear um XOR-split, seja $X_{1}$ o componente. Este componente permite o começo de vários fluxos exclusivos, que se unem num componente XOR-join ou no evento de fim. Seja $u$ o estado que indica que a instância de workflow pode executar $X_{1}$, e seja $m$ 
o número de fluxos exclusivos que saem de $X_{1}$. Então, para mapear $X_{1}$, primeiro criamos um estado $s_{i}$ em $S$, onde $1 \leqslant i \leqslant m$, para cada fluxo de $X_{1}$. Depois, criamos uma transição de $u$ para cada $s_{i}$, cuja taxa será $r \times \mathbb{P}$ (i-ésimo fluxo), isto é, a taxa fixa $r$ multiplicada pela probabilidade do i-ésimo fluxo acontecer.

- No caso de um XOR-join, que faz a junção de fluxo alternativos, primeiro adicionamos um estado $v$ em $S$. Agora, seja $x$ o número de fluxos de entrada e seja $s_{i} \in S$, para $1 \leqslant i \leqslant x$, o último estado do i-ésimo fluxo de entrada. Então, deve-se criar uma transição de $s_{i}$ para $x$, que terá o rótulo null.

Na Figura 4.4 representamos o mapeamento da escolha, onde as flechas tracejadas representam os fluxos, que devem ser mapeados independentemente.

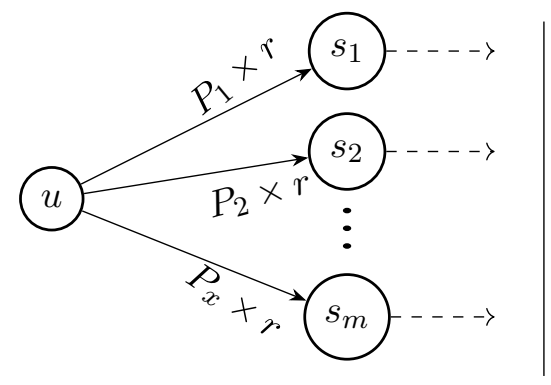

(a)

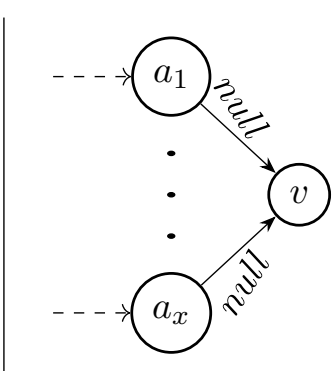

(b)

Figura 4.4: Estados e transições geradas para estruturas de escolha. Em (a) está o XOR-split; e (b) representa o XOR-join.

Para finalizar o processo de mapeamento dos componentes, podemos remover as transições rotuladas como null, já que não representam nenhuma mudança no estado do sistema. Seja a transição $t=(u, v$,null $)$. Para cada estado $w \in S$, tal que existe uma transição de $t^{\prime}=(w, u, x)$, deve ser criada uma nova transição $t^{\prime \prime}=(w, v, x)$. Depois disso, o estado $u$ deve ser removido de $S$ e, portanto, também as transições que tem $u$ como origem ou fim. Esse procedimento é feito até não existir na cadeia de Markov mais nenhuma transição com rótulo null. Com isso, o mapeamento estará completo.

O mapeamento para o workflow da Figura 4.1 é mostrado na Figura 4.5, onde ainda não foram removidas as transições com rótulo null. Já na Figura 4.6 podemos ver os estados e transições finais, depois de aplicar o procedimento de remoção de transições de rótulo null.

Uma vez definidos os conjuntos $S$ e $T$, que representam os estados e transições respectivamente, os quais descrevem o comportamento de uma instância de workflow, a definição da CMTCP subjacente se dá de forma quase direta.

Seja $n=|S|$ o número de estados de uma instância e $N$ o número de instâncias do workflow. Podemos definir a CMTCP $\chi^{(N)}=\left(X^{(N)}, D^{(N)}, T^{(N)}, d_{0}^{(N)}\right)$, onde:

- Para cada estado $s \in S$, temos uma variável $X_{s}^{(N)} \in X^{(N)}$;

- $D^{(N)}$ é definido por $\{0, \cdots, N\}^{n}$, e $d_{0}^{(N)}=\{N, 0, \ldots, 0\}$, ou seja, todas as instâncias estão no estado inicial.

- Para cada transição $t_{i}=(u, v, x)$ em $T$, com $u, v \in S$, temos uma transição $\tau_{i}=\left(a_{i}, s_{u}^{(N)}, t_{v}^{(N)}, x^{(N)}\right)$ em $T^{(N)}$, onde: 


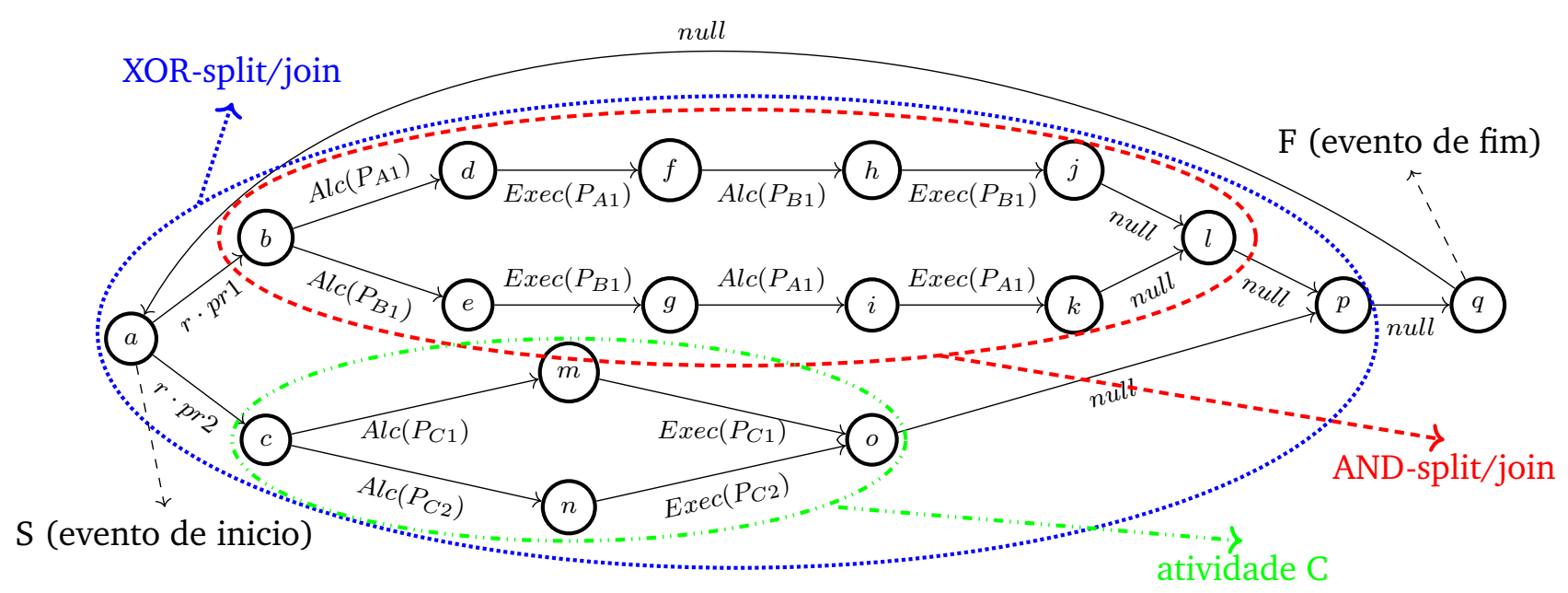

Figura 4.5: Estados (círculos) e transições (flechas), que representam a execução de uma instância do modelo de workflow da Figura 4.1.

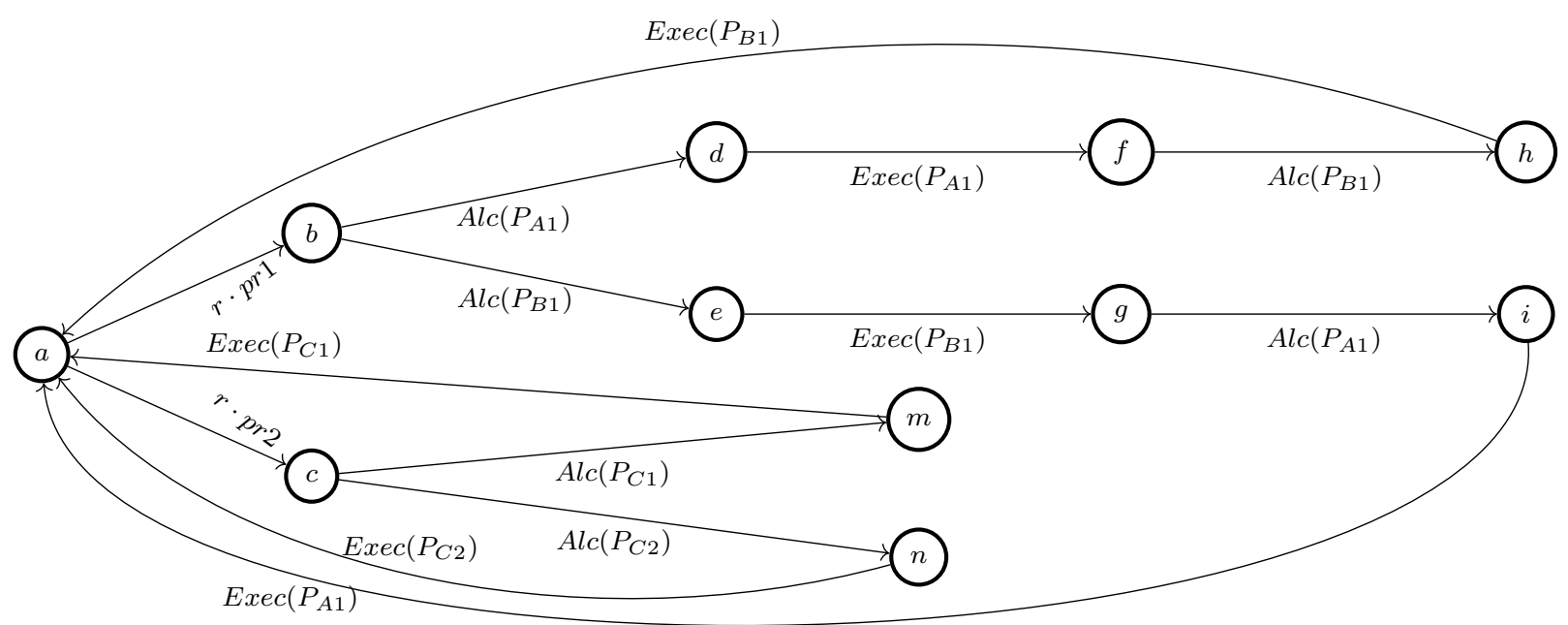

Figura 4.6: Estados (em circulo) e transições (flechas) finais, depois de remover as transições com rótulo null do mapeamento na Figura 4.5.

- $a_{i}$ é o nome da transição;

- $s_{u}^{(N)}$ é um vetor de $n$ elementos, tal que o elemento da posição de $u$ em $S$ é 1 ; o resto dos elementos tem valor 0 ;

- $t_{v}^{(N)}$ é um vetor análogo a $s_{u}$, só que definido para o estado $v$;

- $x^{(N)}=x \cdot X_{u}^{(N)}$, a taxa da transição, que é a soma das taxas de cada instância no estado $u$.

Dessa forma, fica totalmente definida a CMTCP que modela o comportamento de um workflow com $N$ instâncias.

Como exemplo, considere os estados e transições da Figura 4.6, onde $S=(a, b, c, d, e, f, g, h, i, m, n)$, e seja $N$ o número de instâncias. Podemos descrever a seguinte CMTCP correspondente: 
- $X^{(N)}=\left(X_{a}^{(N)}, X_{b}^{(N)}, X_{c}^{(N)}, X_{d}^{(N)}, X_{e}^{(N)}, X_{f}^{(N)}, X_{g}^{(N)}, X_{h}^{(N)}, X_{i}^{(N)}, X_{m}^{(N)}, X_{n}^{(N)}\right)$, como conjunto de variáveis;

- $D^{(N)}=\{0, \cdots, N\}^{1} 1$, e $d_{0}^{(N)}=(N, 0,0,0,0,0,0,0,0,0,0)$;

- $T^{(N)}=\left(\tau_{1}, \cdots, \tau_{14}\right)$, onde cada $\tau_{i}$ é definido como no exemplo abaixo:

Seja $t=\left(d, f, \operatorname{Exec}\left(P_{A 1}\right)\right)$, com $d, f \in S$, a transição correspondente na CMTCP será $\tau_{i}=$ $\left(a_{i}, s_{d}^{(N)}, t_{f}^{(N)}, \operatorname{Exec}\left(P_{A 1}\right) \cdot X_{d}^{(N)}\right)$, onde

- $s_{d}^{(N)}=(0,0,0,1,0,0,0,0,0,0,0) \mathrm{e}$

- $t_{f}^{(N)}=(0,0,0,0,0,1,0,0,0,0,0)$.

\subsubsection{SDTC do modelo de workflow}

Da Definição 4.1.1 para uma CMTCP, sabemos que em essência ela é uma CMTC, então, tem um espaço de estados discreto, o que procuramos evitar neste trabalho. Foi mostrado também que sob algumas condições, as CMTCPs podem ser aproximadas por SDTCs por meio dos Teoremas 4.1.2 e 4.1.3. Na continuação, verificamos as condições nas CMTCPs para workflows.

Seja $N$ o número de instâncias do workflow e seja $\chi^{(N)}=\left(X^{(N)}, D^{(N)}, T^{(N)}, d_{0}^{(N)}\right)$ a CMTCP gerada a partir desse workflow. Definimos o tamanho do sistema por $\gamma_{N}=N$, fazendo com que o incremento básico seja $\delta_{N}=1 / N$. Seja $\hat{\chi}^{(N)}=\left(\hat{X}^{(N)}, \hat{D}^{(N)}, \hat{T}^{(N)}, \hat{d}_{0}^{(N)}\right)$ o modelo normalizado (descrito na Seção 4.1) correspondente a $\chi^{(N)}$ de acordo com $\delta_{N}$ e $\gamma_{N}$.

Quando foram explicadas as condições de escalamento que devem ser verificadas no sistema, para que esse possa ser aproximado por um sistema determinístico (Seção 4.1.2.1), foi mencionado que essas condições são satisfeitas quando os vetores de mudança de estados são independentes de $N$ (no modelo não normalizado). Da forma em que $\chi^{(N)}$ é gerada para os workflows, podemos ver que, para qualquer transição $\tau_{i}=\left(a, s^{(N)}, t^{(N)}, x^{(N)}\right)$ em $T^{(N)}$, os vetores $s^{(N)}$ e $t^{(N)}$ são independentes de $N$, portanto o vetor de mudanças $v^{(N)}=t^{(N)}-s^{(N)}$ também é independente de $N$. Logo, as condições de escalamento são satisfeitas para $\chi^{(N)}$.

Então, seja $\hat{X}^{(N)}$ a CMTC associada à CMTCP $\hat{\chi}^{(N)}$. Pelos Teoremas 4.1.2 e 4.1.3, sabemos que o SDTC $\hat{x}^{(N)}(t)$ é uma boa aproximação para $\hat{X}^{(N)}$, onde $\hat{x}^{(N)}(t)$ é a solução da ODE:

$$
\frac{d \hat{x}^{(N)}(t)}{d t}=F^{(N)}\left(\hat{x}^{(N)}(t)\right)
$$

Então, precisamos calcular $F^{(N)}$ como definido na Equação 4.5, isto é:

$$
F^{(N)}(\hat{d})=\sum_{\tau \in \hat{T}^{(N)}} \hat{v}_{\tau}^{(N)} \hat{r}_{\tau}^{(N)}(\hat{d})
$$

Portanto, podemos aproximar o comportamento de modelos de workflows por meio de SDTCs em forma de ODEs, evitando assim o problema da explosão de estados dos modelos Markovianos.

Para o workflow de exemplo, na Figura 4.1, para cada transição $\tau_{i} \in T^{(N)}$, temos que a função $F$ é dada por: 


$$
\begin{aligned}
F^{(N)}\left(x^{(N)}(t)\right)= & (-1,1,0,0,0,0,0,0,0,0,0) \cdot r \cdot \operatorname{pr} 1 \cdot X_{a}^{(N)}+ \\
& (-1,0,1,0,0,0,0,0,0,0,0) \cdot r \cdot \operatorname{pr} 2 \cdot X_{a}^{(N)}+ \\
& (0,-1,0,1,0,0,0,0,0,0,0) \cdot \operatorname{Alc}\left(P_{A 1}\right) \cdot X_{b}^{(N)}+ \\
& (0,-1,0,0,1,0,0,0,0,0,0) \cdot \operatorname{Alc}\left(P_{B 1}\right) \cdot X_{b}^{(N)}+ \\
& (0,0,-1,0,0,0,0,0,0,1,0) \cdot \operatorname{Alc}\left(P_{C 1}\right) \cdot X_{c}^{(N)}+ \\
& (0,0,-1,0,0,0,0,0,0,0,1) \cdot \operatorname{Alc}\left(P_{C 2}\right) \cdot X_{c}^{(N)}+ \\
& (0,0,0,-1,0,1,0,0,0,0,0) \cdot \operatorname{Exec}\left(P_{A 1}\right) \cdot X_{d}^{(N)}+ \\
& (0,0,0,0,-1,0,1,0,0,0,0) \cdot \operatorname{Exec}\left(P_{B 1}\right) \cdot X_{e}^{(N)}+ \\
& (0,0,0,0,0,-1,0,1,0,0,0) \cdot \operatorname{Alc}\left(P_{B 1}\right) \cdot X_{f}^{(N)}+ \\
& (0,0,0,0,0,0,-1,0,1,0,0) \cdot \operatorname{Alc}\left(P_{A 1}\right) \cdot X_{g}^{(N)}+ \\
& (1,0,0,0,0,0,0,-1,0,0,0) \cdot \operatorname{Exec}\left(P_{B 1}\right) \cdot X_{h}^{(N)}+ \\
& (1,0,0,0,0,0,0,0,-1,0,0) \cdot \operatorname{Exec}\left(P_{A 1}\right) \cdot X_{i}^{(N)}+ \\
& (1,0,0,0,0,0,0,0,0,-1,0) \cdot \operatorname{Exec}\left(P_{C 1}\right) \cdot X_{m}^{(N)}+ \\
& (1,0,0,0,0,0,0,0,0,0,-1) \cdot \operatorname{Exec}\left(P_{C 2}\right) \cdot X_{n}^{(N)}
\end{aligned}
$$

Consequentemente, $F$ determina as ODEs da forma $\frac{d x_{s}^{(\hat{N})}(t)}{d t}$, para cada $s \in S$, as quais descrevem o SDTC e são a aproximação determinística do comportamento do workflow. Por exemplo, para o estado $a$ temos que:

$$
\begin{aligned}
\frac{d \hat{x}_{a}^{(N)}(t)}{d t}= & -r \cdot \operatorname{pr} 1 \cdot X_{a}^{(N)}-r \cdot \operatorname{pr} 2 \cdot X_{a}^{(N)}+\operatorname{Exec}\left(P_{B 1}\right) \cdot X_{h}^{(N)} \\
& +\operatorname{Exec}\left(P_{A 1}\right) \cdot X_{i}^{(N)}+\operatorname{Exec}\left(P_{C 1}\right) \cdot X_{m}^{(N)}+\operatorname{Exec}\left(P_{C 2}\right) \cdot X_{n}^{(N)}
\end{aligned}
$$

Neste capítulo foi descrita a Teoria do Campo Médio, que procura aproximar sistemas estocásticos que atuam em espaços de estado discretos, por um sistema determinístico com espaço de estados contínuo, evitando assim o problema da explosão de estados. Pelos Teoremas 4.1.2 e 4.1.3, sabemos que a aproximação determinística existe quando o sistema estocástico está sujeito às condições de escalamento. Então, a partir dessas definições, mostramos o procedimento que é proposto neste trabalho para determinar o SDTC para um workflow. Com isso, podemos analisar o desempenho do workflow por meio da aproximação determinística. 


\section{Capítulo 5}

\section{Extração de métricas de desempenho a partir de ODEs}

A análise preditiva de desempenho com modelos analíticos tinha sido declarada como impraticável no Capítulo 3, devido ao crescimento exponencial dos estados presente nos modelos Markovianos. Nesses modelos, as medidas de desempenho são calculadas com base em funções de indexação que são verificadas em cada possível estado. Além disso, o cálculo do estado estacionário do sistema demanda um tempo computacional inviável.

No Capítulo 4 foi demonstrada a existência de um sistema determinístico de tempo contínuo (SDTC) que é uma aproximação do comportamento de workflows com o tipo de características definidas neste trabalho. O SDTC é descrito por meio de um conjunto de ODEs, cuja solução representa o estado do sistema em um dado tempo.

Em comparação com as RPEGs, podemos interpretar a solução das ODEs em um tempo $t$ como sendo a distribuição de fichas nos lugares da RPEG em $t$, porém considerando somente os lugares que não são usados para representar os recursos. Como mencionado anteriormente, o mapeamento do modelo BPMN do workflow para RPEGs e para a CMTCPs segue um procedimento similar.

Para ilustrar o problema, considere o workflow com estrutura sequencial de atividades na Figura 5.1, com parâmetros: $A R_{1}=4, B R_{1}=2, r=500, I=50$ e $N R_{1}=100$. A Figura 5.2 mostra a comparação do comportamento estocástico (do mapeamento para RPEG, com a ferramenta GreatSPN), com o comportamento da aproximação determinística (do mapeamento para ODEs, com um script feito em python).

Para esse modelo de workflow de estrutura relativamente simples, podemos ver na Figura 5.2 o alto consumo de tempo para calcular soluções estocásticas, mesmo que para um número de instâncias na ordem de dezenas. Por outro lado, vemos que o sistema determinístico oferece uma solução computada muito mais rapidamente e que tem um comportamento similar ao estocástico, como detalhado na Tabela 5.1.

Além disso, vimos que no sistema determinístico, é possível determinar novas variáveis com relativa facilidade, como é o caso da variável $X_{R 1}$ no exemplo, e que são calculadas em tempos razoáveis. Isso já nos dá uma noção de que, por meio das variáveis das ODEs, é possível criar novas funções que descrevam medidas de desempenho do sistema. Continuando com o exemplo, a variável criada $X_{R 1}$ representa a quantidade de recursos $R 1$ que não estão sendo utilizados; dessa forma, podemos calcular a utilização de recursos sem muita dificuldade.

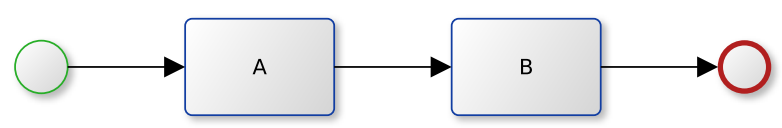

Figura 5.1: Workflow com estrutura sequencial de atividades

A seguir, descreveremos algumas funções para o cálculo de índices de desempenho geralmente 


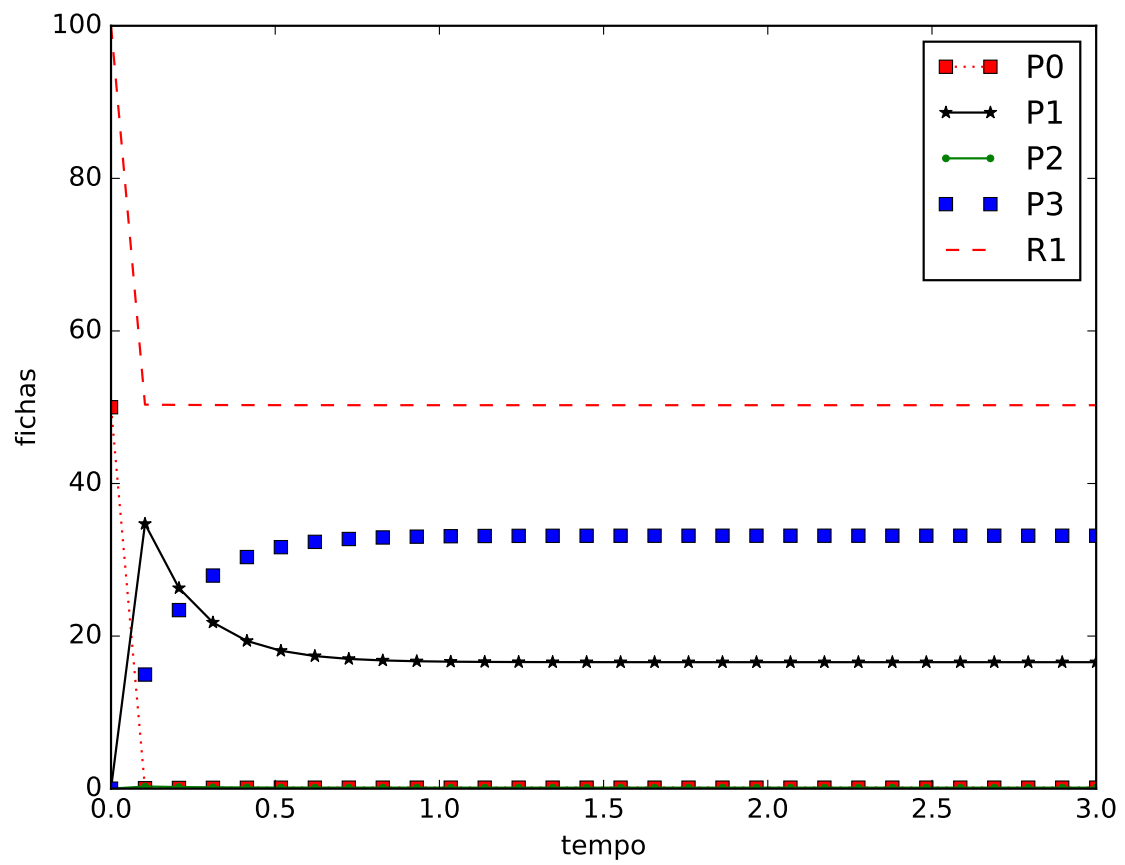

(a) Trajetórias estocásticas do valor médio de fichas nos lugares do workflow da Figura 5.1. Solução gerada em 869 segundos.

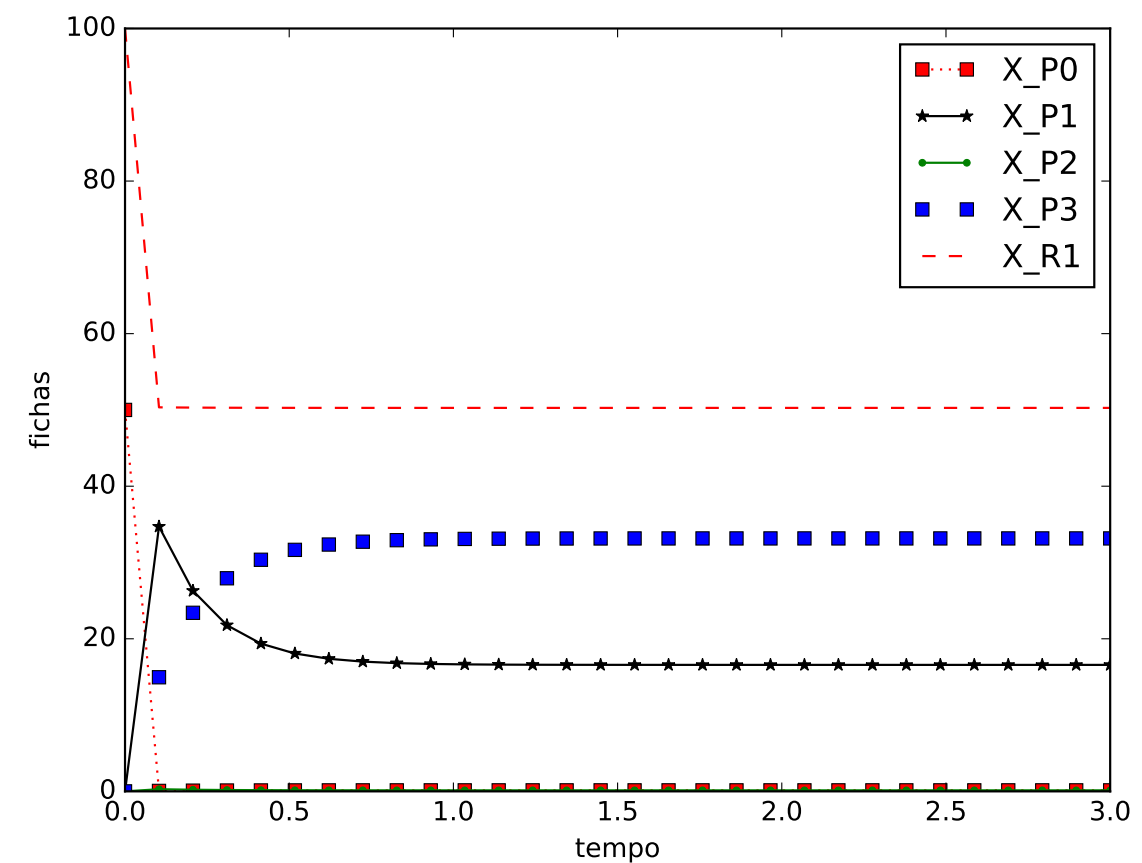

(b) Trajetórias determinísticas do SDTC mapeado para o workflow da Figura 5.1. Solução obtida em 0.163 segundos.

Figura 5.2: Comparação dos comportamentos estocástico e determinístico do workflow da Figura 5.1. Em (b), das ODEs calculamos $X_{R 1}(t)=N R_{1}-\left(X_{P 1}(t)+X_{P 3}(t)\right)$.

requisitados. Tais funções serão definidas para as variáveis do conjunto de ODEs. Em outras palavras, descreveremos a forma de obter métricas de desempenho para workflows por meio da sua aproximação determinística.

\section{1 Índices de Desempenho}

Primeiramente, para todas as atividades do workflow, sejam os seguintes estados da CMTCP subjacente ao workflow: $u$ indica que uma instância pode executar alguma atividade, $\left(s_{0}, \cdots, s_{i}\right)$ 


\begin{tabular}{|l|c|c|c|}
\hline Lugar - Variável & Estocástico & Determinístico & Erro (\%) \\
\hline$P 0-X \_P 0:$ tarefa A está habilitada & 0.13263 & 0.13263 & 0 \\
\hline$P 1-X \_P 1:$ tarefa A está sendo executada & 16.5783 & 16.5782 & 0.0006 \\
\hline$P 2-X \_P 2:$ tarefa B está habilitada & 0.13263 & 0.13263 & 0 \\
\hline$P 3-X \_P 3:$ tarefa B está sendo executada & 33.1564 & 33.15649 & 0.00027 \\
\hline$R 1-X \_R 1:$ recursos disponíveis & 50.2653 & 50.2653 & 0 \\
\hline
\end{tabular}

Tabela 5.1: Valores das populações da Figura 5.2. Onde o erro porcentual foi calculado como $\frac{\mid \text { Determinístico-Estocástico } \mid}{\text { Estocástico }} \times 100 \%$

indicam que a instância alocou recursos para executar alguma atividade de acordo com os requisitos da atividade e, por último, $v$ que indica que alguma atividade foi executada. Portanto, na aproximação determinística da CMTCP descrita por ODEs, existe uma variável para cada um dos estados definidos anteriormente, i.e., existem as variáveis $X_{u}, X_{s_{0}}, \cdots, X_{s_{i}}$ e $X_{v}$. Em seguida definimos as medidas de desempenho definidas sobre SDTCs.

\subsubsection{Número de Unidades dos Recursos em Uso}

Dado que os estados $\left(s_{0}, \cdots, s_{i}\right)$ representam a alocação de recursos, é possível saber, pela etapa de mapeamento, quais deles alocaram certo tipo de recursos. Então, seja o recurso $R_{1}$, definimos sua utilização como:

$$
\operatorname{Utilizacao}(R 1)=\sum_{s_{i} \text { aloca } R_{1}} X_{s_{i}}
$$

\subsubsection{Tamanho da Fila dos Recursos}

Seguindo o mesmo procedimento, é fácil ver que $u$ representa o estado em que uma entidade espera até poder alocar os recursos necessários para executar alguma atividade. Portanto, o tamanho da fila para o recurso $R_{1}$ será:

$$
\operatorname{Fila}\left(R_{1}\right)=\sum_{u \text { tenta alocar } R_{1}} X_{u}
$$

\subsubsection{Rendimento de Recursos}

O rendimento de um recurso é a quantidade de trabalho executada pelas unidades do recurso em uso. Então, para um recurso $R_{1}$, temos:

$$
\text { Rendimento }\left(R_{1}\right)=\sum_{s_{i} \text { aloca } R_{1}} X_{s_{i}} \cdot Q t\left(s_{i}, R_{1}\right)
$$

onde $Q t\left(s_{i}, R_{1}\right)$ é a quantidade de trabalho que $R_{1}$ faz na atividade para a qual foi mapeada $s_{i}$.

\subsubsection{Rendimento de Atividades}

O rendimento de uma determinada atividade indica o número de instâncias por unidade de tempo que a atividade executa. Seja a atividade $A$, cujos requisitos de recursos são $P_{A}=\left(P_{A 0} \vee\right.$ $\left.\cdots \vee P_{A i}\right)$, definimos o rendimento da atividade como:

$$
\operatorname{Rendimento}(A)=\sum_{j=0}^{i} X_{s_{j}} \cdot \operatorname{Exec}\left(P_{A j}\right)
$$

onde $\operatorname{Exec}\left(P_{A j}\right)$ denota a taxa de execução de $A$ com os recursos especificados em $P_{A j}$. 


\subsubsection{Rendimento do Sistema}

O rendimento do sistema, também conhecido como rendimento do processo, é o número de instâncias completadas por unidade de tempo.

Seja $X_{a}$ uma variável das ODEs, tal que $a$ é o estado incial da CMTCP. Depois, seja o conjunto de variáveis $C=\left(X_{s}\right)$, tal que existe uma transição $t=(s, a, \alpha)$ na CMTCP. Então, o rendimento do sistema será:

$$
\text { RendimentoSistema }=\sum_{X_{s} \in C} X_{s} \cdot \alpha
$$

É necessário esclarecer que essas não são as únicas medidas de desempenho que podem ser calculadas com as soluções das ODEs. Elas são as mais comuns e que foram definidas formalmente, para assim também permitir a visualização de novas funções para medir o desempenho do sistema.

\subsection{Alguns Resultados de Soluções Estocásticas e Determinísticas para Workflows}

\section{Estrutura Sequencial}

Para o workflow de estrutura sequencial (consideramos sequências de atividades), foram comparados os comportamentos estocásticos e determinísticos. Na Tabela 3.5 mostramos o crescimento do espaço de estados e o consumo de tempo e memória para gerá-lo. O número máximo de instâncias é 500, já que para esse número de instâncias não foi possível determinar o estado estacionário da CMTC subjacente, impedindo a análise de desempenho em um processo estocástico dessa escala.

Todavia, para se determinar o comportamento ao longo do tempo, os limites são ainda inferiores. O número máximo de instâncias que puderam ser modeladas foi 50, cujo comportamento é mostrado na Figura 5.2. Para um número maior de instâncias, os tempos de processamento são inviáveis, além de consumir grandes quantidades de memória.

\section{Estrutura de Escolha}

Nos experimentos da estrutura de escolha, do workflow da Figura 5.3, a análise do comportamento estocástico tem limites ainda menores que os da estrutura sequencial. Um fato interessante observado é que, neste tipo de estrutura, quanto menor a escala do modelo, em número de instâncias e quantidade de recursos, piores parecem os resultados obtidos da aproximação determinística.

Na Figura 5.5, os experimentos foram para 3 instâncias e duas unidades de um só tipo de recurso, com parâmetros: $A R_{1}=4, B R_{1}=2, r=500, p r 1=400, p r 2=100, I=3$ e $N R_{1}=2$. Se compararmos as trajetórias, elas são semelhantes, mas podemos ver que existe certa diferença mesmo para tempos pequenos. As trajetórias que mais parecem divergir são as referentes à escolha probabilística, que são os estados $P_{1}$ e $P_{3}$. Porém, quando olhamos esses estados para um sistema de maior escala, na Figura 5.6, que tem 30 instâncias e 20 recursos (a maior escala que conseguimos experimentar para este tipo de estrutura modelada como um sistema estocástico), a divergência das trajetórias estocásticas e determinísticas parece aumentar nesses estados mencionados anteriormente $\left(P_{1}\right.$ e $P_{3}$ ). No sistema determinístico os valores dos lugares depois da escolha são proporcionais às probabilidades de cada possível fluxo, já na solução estocástica não observamos o mesmo comportamento. Cabe ressaltar que essa diferença se dá quando temos mais instâncias que unidades de recursos, pois de outra forma os resultados ficam iguais. 


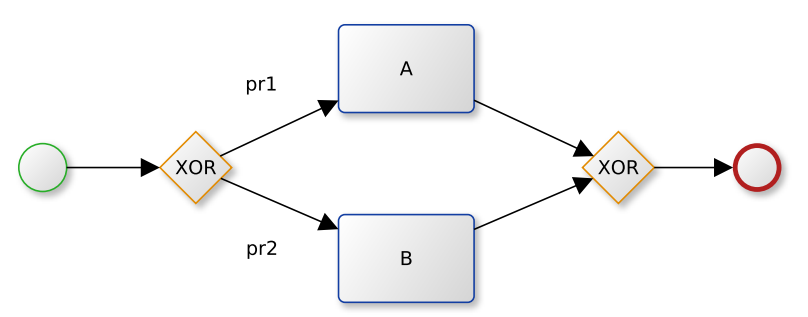

Figura 5.3: Modelo BPMN com estrutura de escolha

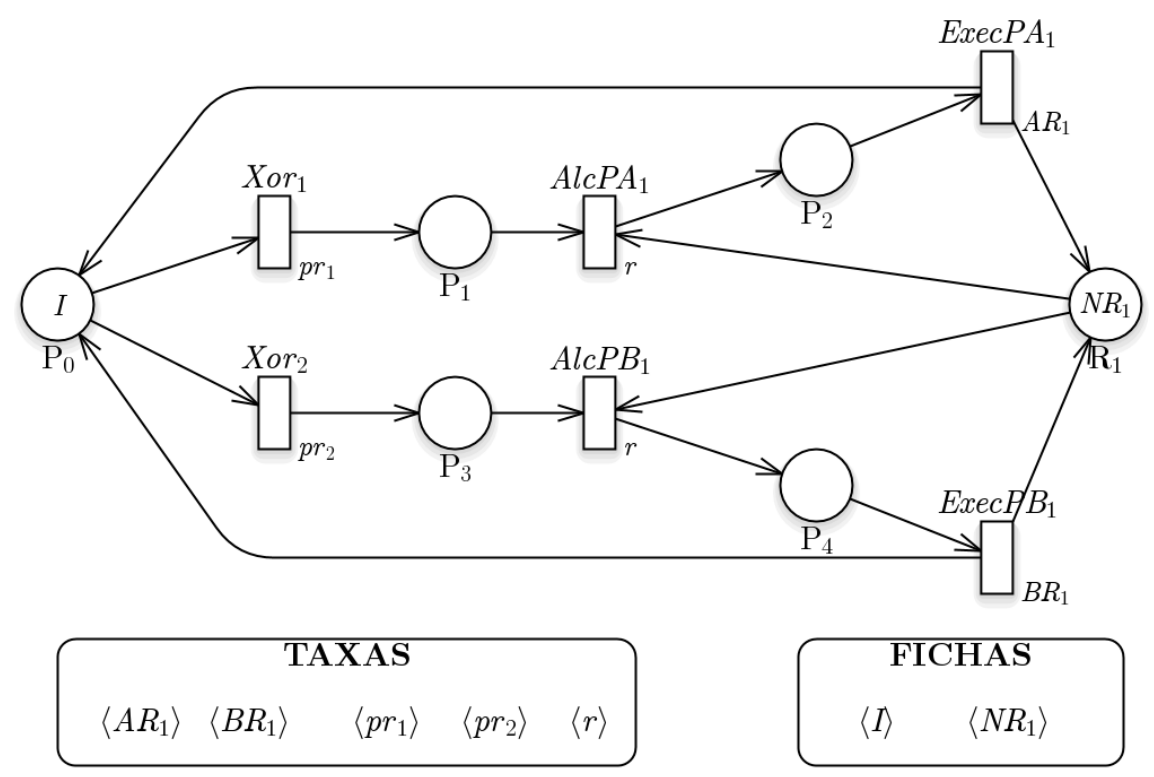

Figura 5.4: RPEG da estrutura de escolha mostrada na Figura 5.3

\begin{tabular}{|l|c|c|c|}
\hline Lugar - Variável & Estocástico & Determinístico & Erro (\%) \\
\hline$P 0-X \_P 0:$ escolha exclusiva & 0.01325 & 0.01325 & 0 \\
\hline$P 1-X \_P 1:$ tarefa A está habilitada & 0.79974 & 0.79999 & 0.03126 \\
\hline$P 2-X_{-} P 2$ : tarefa A está sendo executada & 1.32516 & 1.3247 & 0.03471 \\
\hline$P 3-X_{-} P 3:$ tarefa B está habilitada & 0.19927 & 0.2 & 0.36634 \\
\hline$P 4-X_{-} P 4:$ tarefa B está sendo executada & 0.66258 & 0.66205 & 0.07999 \\
\hline$R 1-X_{-} R 1:$ recursos disponíveis & 0.01226 & 0.01325 & 8.07504 \\
\hline
\end{tabular}

Tabela 5.2: Valores das populações das trajetórias da Figura 5.5.

\begin{tabular}{|l|c|c|c|}
\hline Lugar - Variável & Estocástico & Determinístico & Erro (\%) \\
\hline$P 0-X_{-} P 0:$ escolha exclusiva & 0.13263 & 0.13246 & 0.12818 \\
\hline$P 1-X_{-} P 1:$ tarefa A está habilitada & 9.52476 & 8 & 16.00838 \\
\hline$P 2-X_{-} P 2:$ tarefa A está sendo executada & 13.2625 & 13.24702 & 0.11672 \\
\hline$P 3-X_{-} P 3:$ tarefa B está habilitada & 0.44872 & 2 & 345.71225 \\
\hline$P 4-X_{-} P 4:$ tarefa B está sendo executada & 6.6314 & 6.62052 & 0.16407 \\
\hline$R 1-X_{-} R 1:$ recursos disponíveis & 0.1061 & 0.01325 & 899.69811 \\
\hline
\end{tabular}

Tabela 5.3: Valores das populações das trajetórias da Figura 5.6.

\section{Estrutura de Paralelismo}

Para a estrutura de paralelismo, descrito na Figura 5.7, temos um maior espaço de estados (se comparado ao das outras estruturas básicas) para seu modelo Markoviano (RPEG da Figura 5.8). Como consequência, os limites de escalamento para produzir resultados experimentais estocásticos foram mais baixos, na ordem de poucas dezenas. 


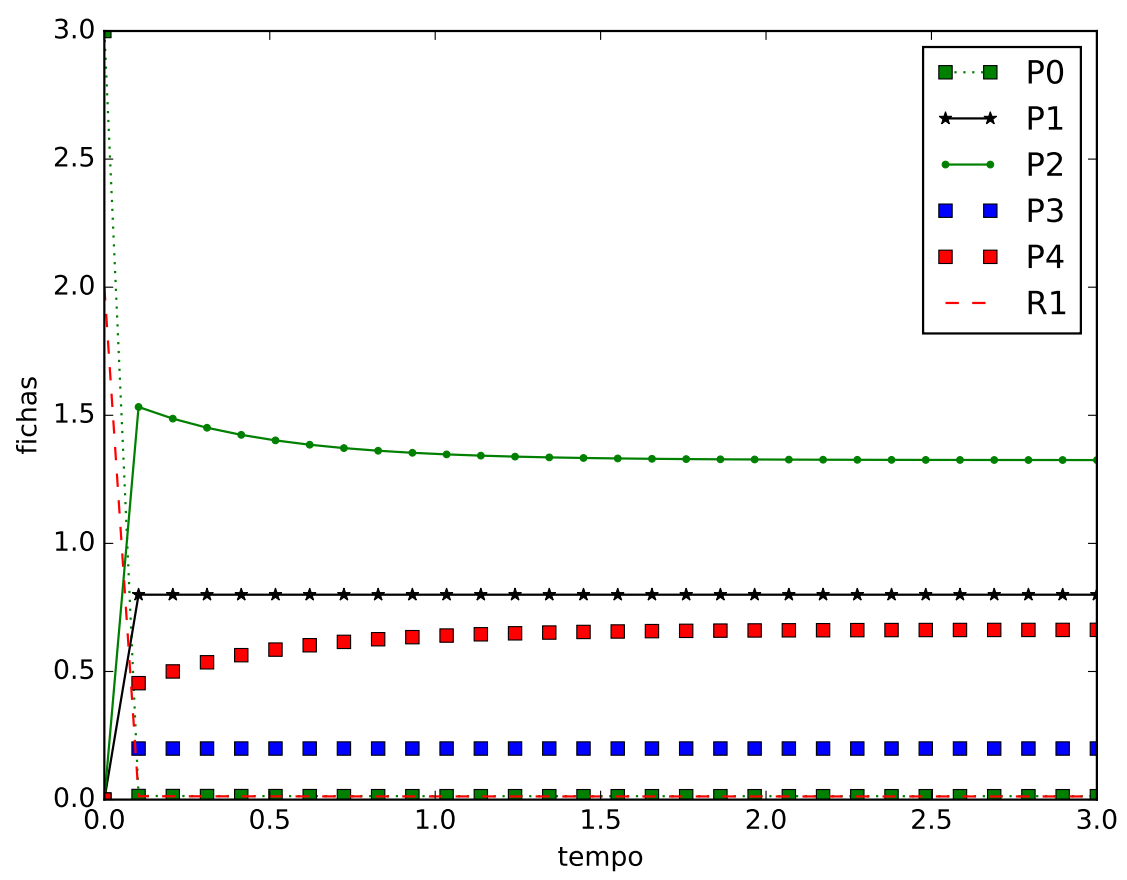

(a) Trajetórias estocásticas do valor médio de fichas nos lugares da RPEG da Figura 5.4 Solução gerada em 0.383 segundos.

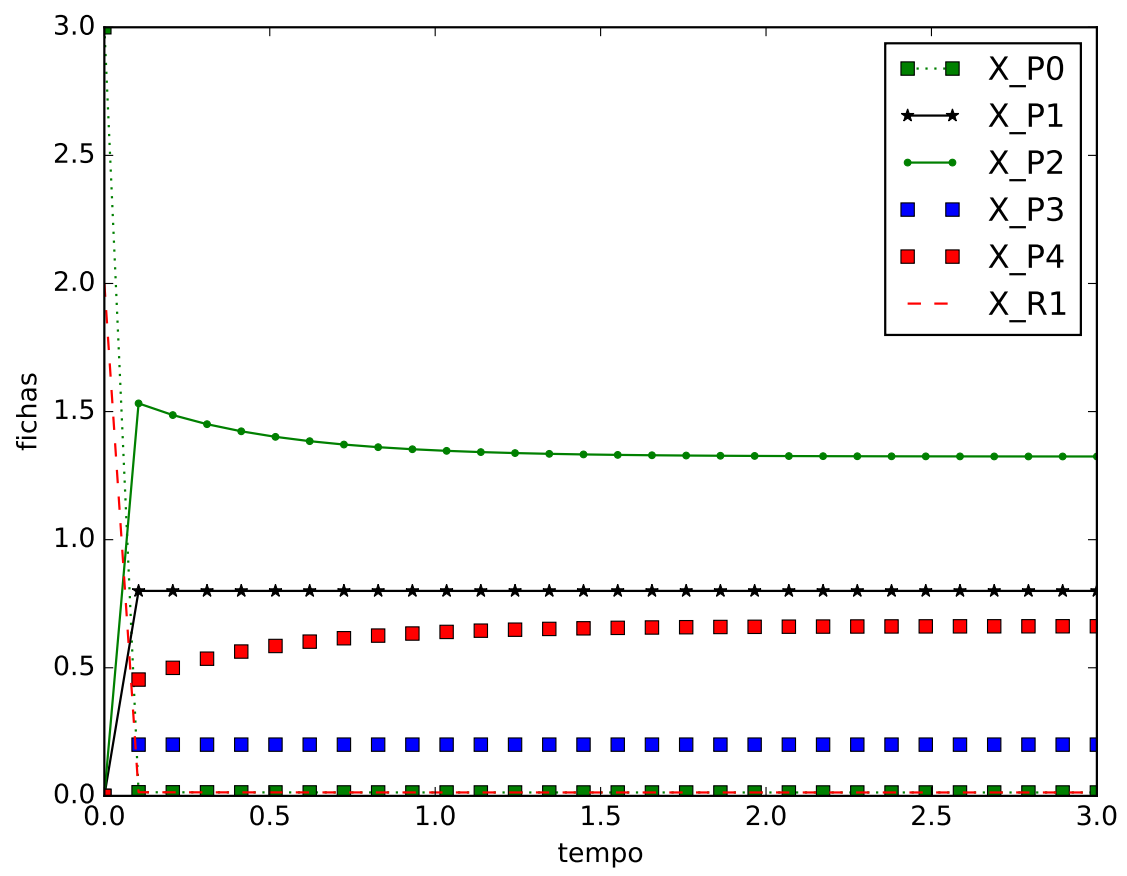

(b) Trajetórias determinísticas do SDTC mapeado para o workflow da Figura 5.3. Solução obtida em 0.155 segundos.

Figura 5.5: Comparação dos comportamentos estocástico e determinístico do workflow da Figura 5.3 para $I=3$ e $N R_{1}=2$. Em (b) temos que $X_{R 1}(t)=N R_{1}-\left(X_{P 2}(t)+X_{P 4}(t)\right)$.

Na Figura 5.9, comparamos a evolução do sistema ao longo do tempo, para 10 instâncias de workflow e 10 unidades de recurso. Os parâmetros são $A R_{1}=4, B R_{1}=2, r=500, I=10 \mathrm{e}$ $N R_{1}=10$. Os resultados da aproximação são muito semelhantes aos do método estocástico, pois as trajetórias estocásticas e determinísticas seguem uma evolução em um tempo similar.

Para um escalamento maior do mesmo sistema, na Figura 5.10, com 15 instâncias e 10 unidades de recurso, os resultados das trajetórias são similares. É possível observar que nos tempos iniciais 


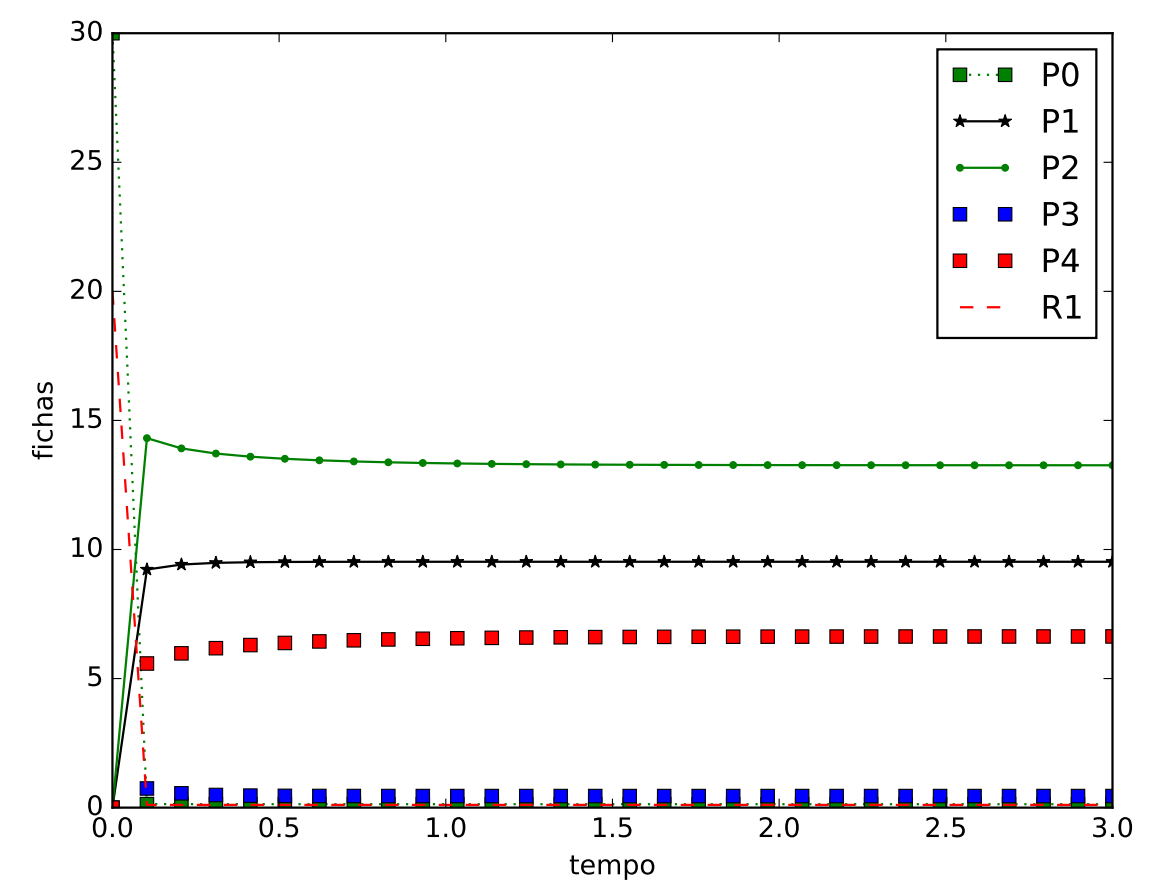

(a) Trajetórias estocásticas do valor médio de fichas nos lugares da RPEG da Figura 5.4. Solução gerada em 1193 segundos.

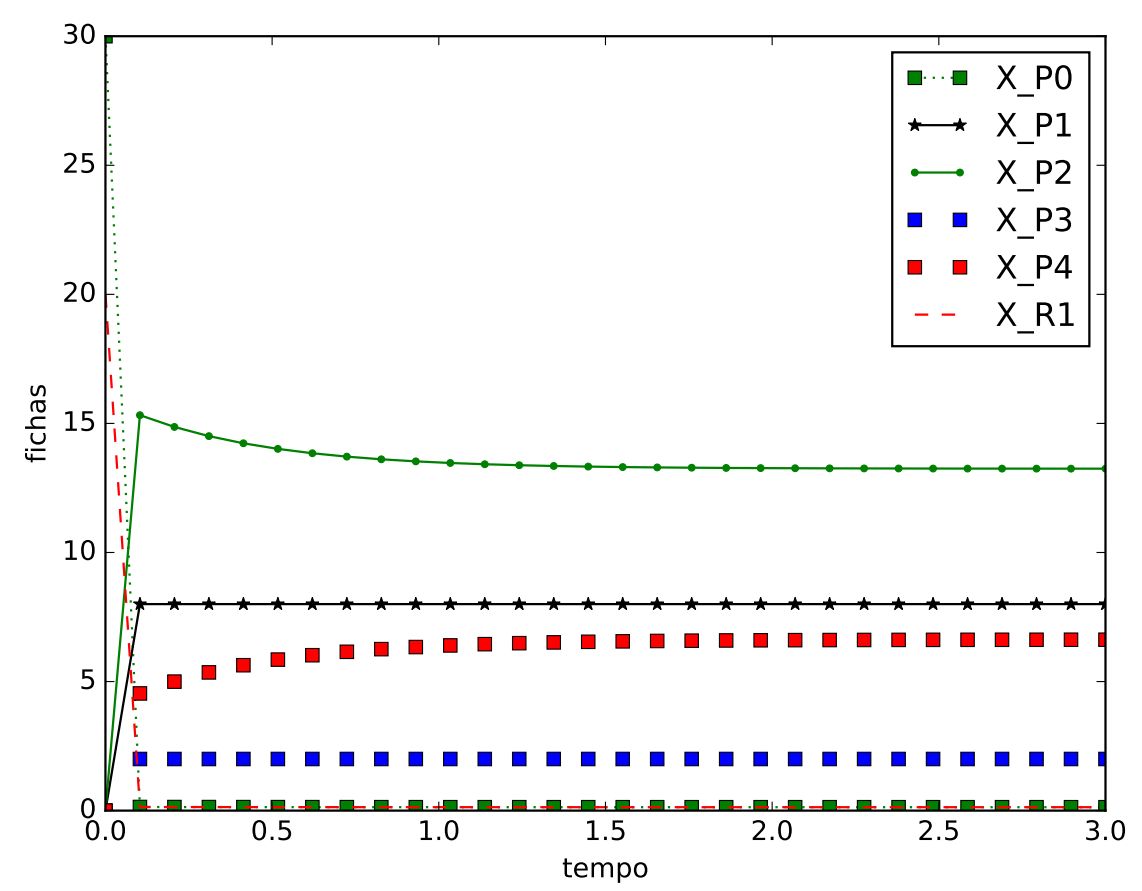

(b) Trajetórias determinísticas do SDTC mapeado para o workflow da Figura 5.3. Solução obtida em 0.162 segundos.

Figura 5.6: Comparação dos comportamentos estocástico e determinístico similar à Figura 5.5, quando I = 30 e $N R_{1}=20$.

a diferença entra as trajetórias é maior, no entanto, elas convergem a medida que evoluem no tempo. Cabe ressaltar que para escalas maiores, no número de instâncias e recursos, os tempos de cômputo das soluções estocásticas não são razoáveis, tornando a adição de mais componentes no modelo estocástico impraticável.

Para o modelo de workflow da Figura 5.11, o qual contém as três estruturas básicas antes mencionadas, podemos observar os resultados das soluções Markovianas e do respectivo conjunto 


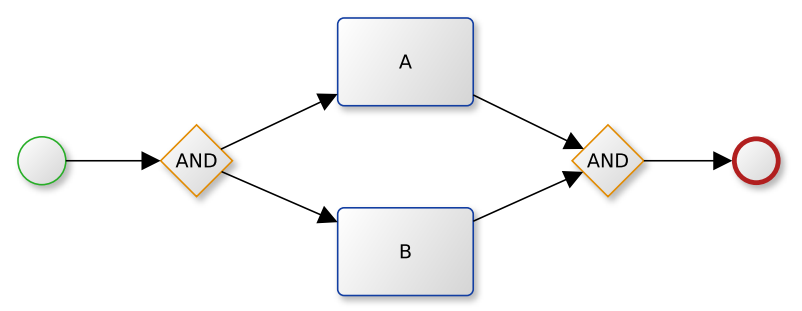

Figura 5.7: Modelo BPMN com estrutura paralela

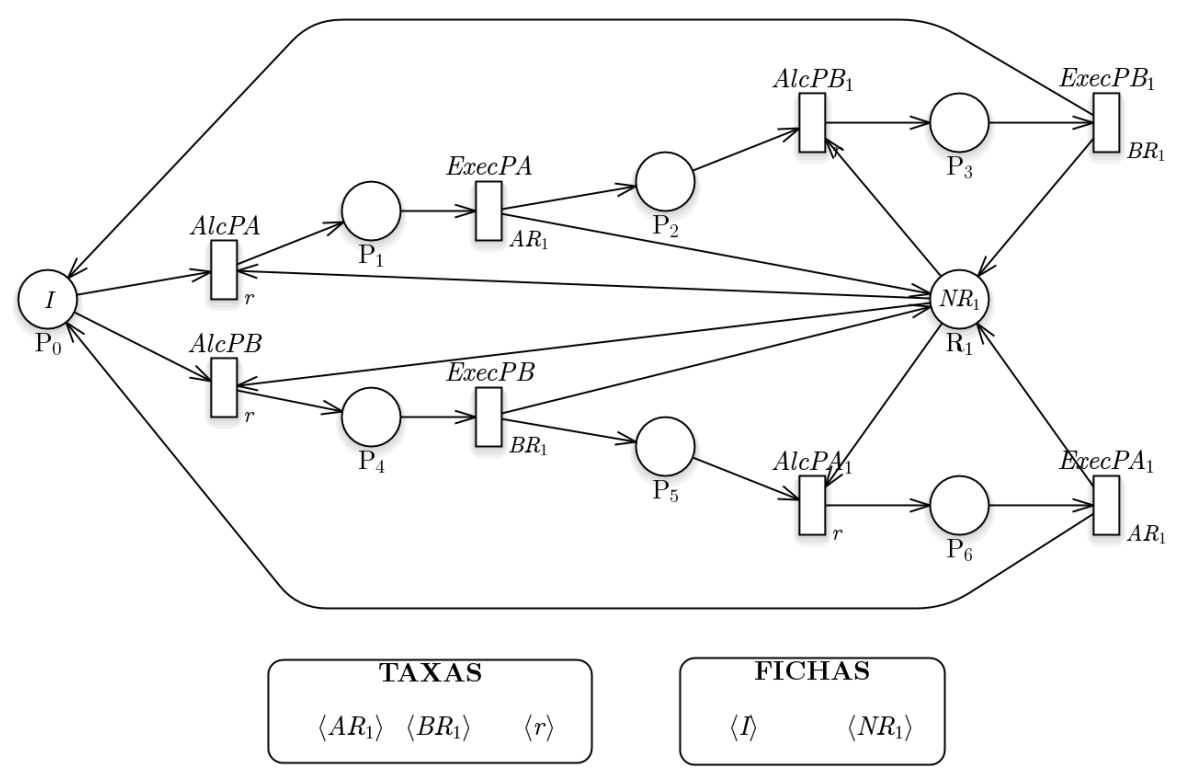

Figura 5.8: RPEG modelando a estrutura paralela mostrada na Figura 5.7

\begin{tabular}{|l|c|c|c|}
\hline Lugar - Variável & Estocástico & Determinístico & Erro (\%) \\
\hline$P 0-X \_P 0:$ início paralelismo & 0.01328 & 0.01328 & 0 \\
\hline$P 1-X \_P 1:$ tarefa A está sendo executada & 1.66003 & 1.66003 & 0 \\
\hline$P 2-X \_P 2:$ tarefa B está habilitada & 0.01328 & 0.01328 & 0 \\
\hline$P 3-X \_P 3:$ tarefa B está sendo executada & 3.32009 & 3.32005 & 0.0012 \\
\hline$P 4-X \_P 4:$ tarefa B está sendo executada & 3.32003 & 3.32005 & 0.0006 \\
\hline$P 5-X \_P 5:$ tarefa A está habilitada & 0.01328 & 0.01328 & 0 \\
\hline$P 6-X \_P 6:$ tarefa A está sendo executada & 1.66001 & 1.66003 & 0.0012 \\
\hline$R 1-X \_R 1$ : recursos disponíveis & 0.03984 & 0.03984 & 0 \\
\hline
\end{tabular}

Tabela 5.4: Valores das populações das trajetórias da Figura 5.9.

\begin{tabular}{|l|c|c|c|}
\hline Lugar - Variável & Estocástico & Determinístico & Erro (\%) \\
\hline$P 0-X \_P 0:$ início paralelismo & 1.67235 & 1.67987 & 0.44967 \\
\hline$P 1-X \_P 1:$ tarefa A está sendo executada & 1.66367 & 1.66006 & 0.21699 \\
\hline$P 2-X \_P 2:$ tarefa B está habilitada & 1.67315 & 1.67981 & 0.39805 \\
\hline$P 3-X \_P 3:$ tarefa B está sendo executada & 3.32803 & 3.31899 & 0.27163 \\
\hline$P 4-X \_P 4:$ tarefa B está sendo executada & 3.32724 & 3.32047 & 0.20347 \\
\hline$P 5-X \_P 5:$ tarefa A está habilitada & 1.67225 & 1.68017 & 0.47361 \\
\hline$P 6-X \_P 6:$ tarefa A está sendo executada & 1.66332 & 1.66063 & 0.16172 \\
\hline$R 1-X \_R 1:$ recursos disponíveis & 0.01775 & 0.03985 & 124.50704 \\
\hline
\end{tabular}

Tabela 5.5: Valores das populações das trajetórias da Figura 5.10.

de ODEs na Figura 5.13, com parâmetros: $A R_{1}=2, B R_{1}=4, C R_{1}=1, D_{R} 1=3, r=500$, $\operatorname{pr} 1=400, \operatorname{pr} 2=100, I=5$ e $N R_{1}=5$. É possível notar a similaridade das duas soluções nessa 


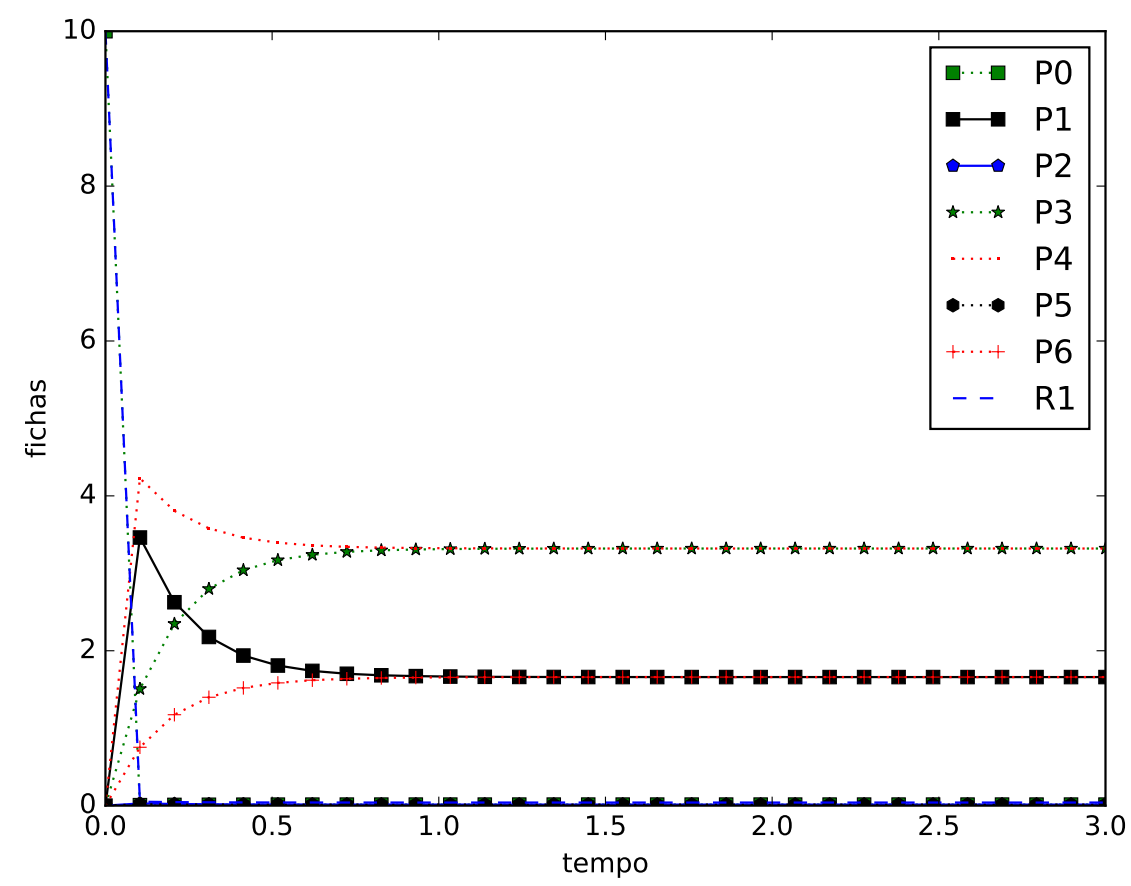

(a) Trajetórias estocásticas do valor médio de fichas nos lugares da RPEG da Figura 5.8. Solução gerada em 164.194 segundos.

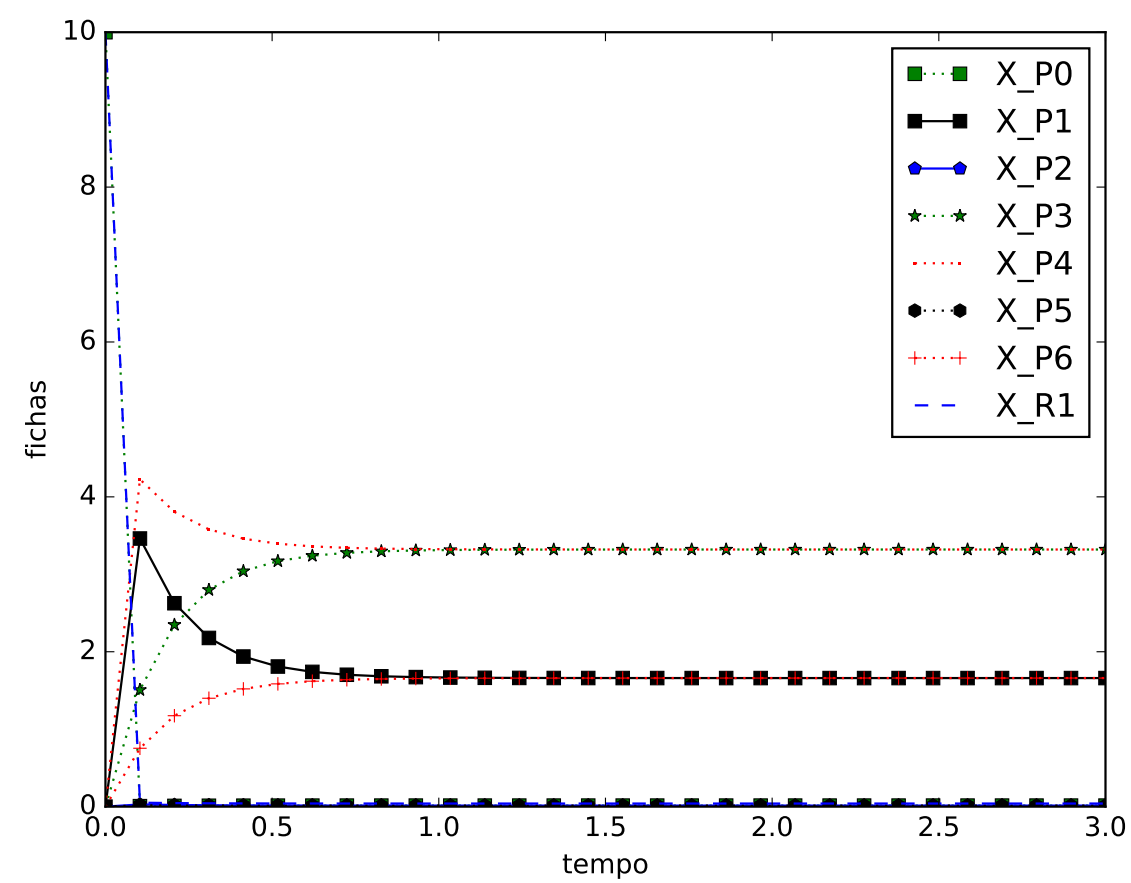

(b) Trajetórias determinísticas do SDTC mapeado para o workflow da Figura 5.7. Solução obtida em 0.166 segundos.

Figura 5.9: Comparação dos comportamentos estocástico e determinístico do workflow da Figura 5.7. Em (b) temos que $X_{R 1}(t)=N R_{1}-\left(X_{P 1}(t)+X_{P 3}(t)+X_{P 4}(t)+X_{P 6}(t)\right)$.

configuração.

Quando aumentamos o número de instâncias, ou seja, agora temos $I=10$, os resultados dos dois tipos de soluções têm maior diferença, se comparados com os da configuração anterior. Na Tabela 5.6 vemos os valores finais, no estado estacionário, de cada estado do workflow. Podemos observar que os estados com maior diferença nas aproximações são aqueles referentes ao componente de escolha ( $P 1$ e $P 8$ ), como descrito na estrutura de escolha, contudo, agora não são mais 


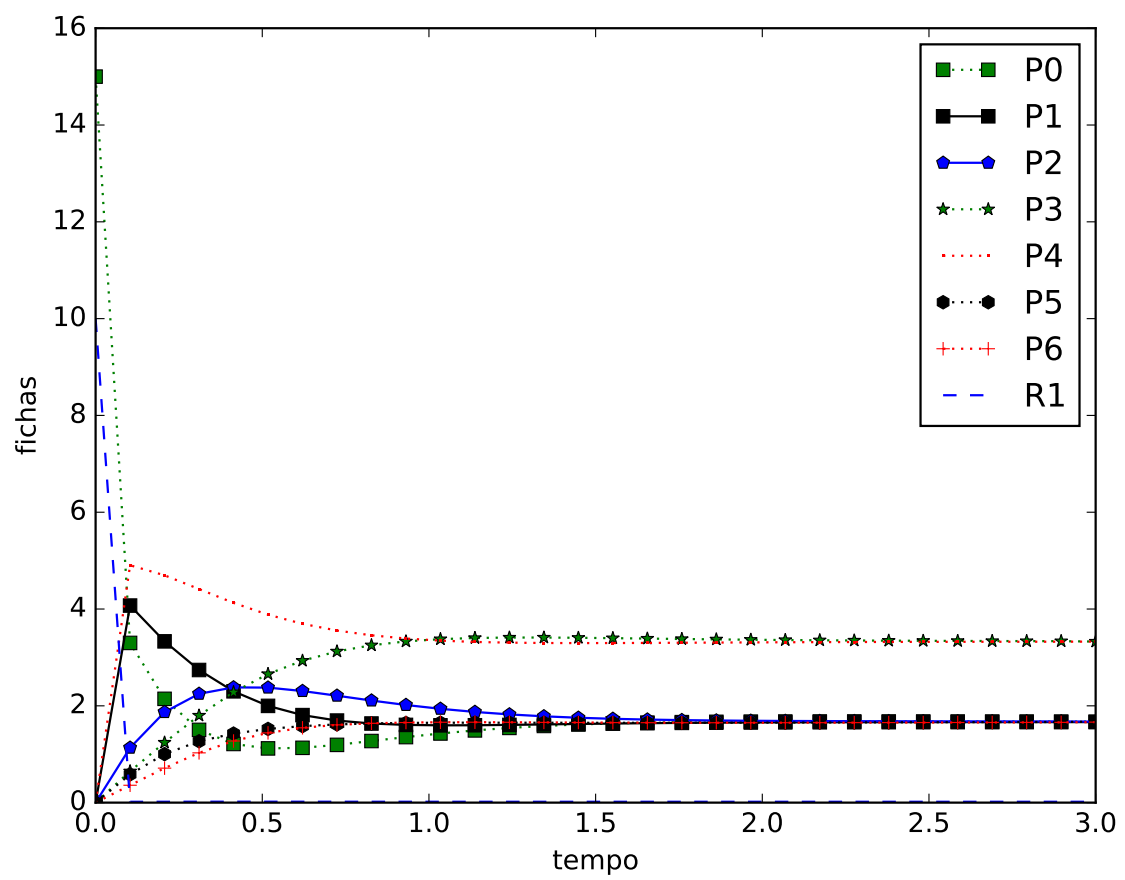

(a) Trajetórias estocásticas do valor médio de fichas nos lugares da RPEG da Figura 5.8. Solução gerada em 1136.555 segundos.

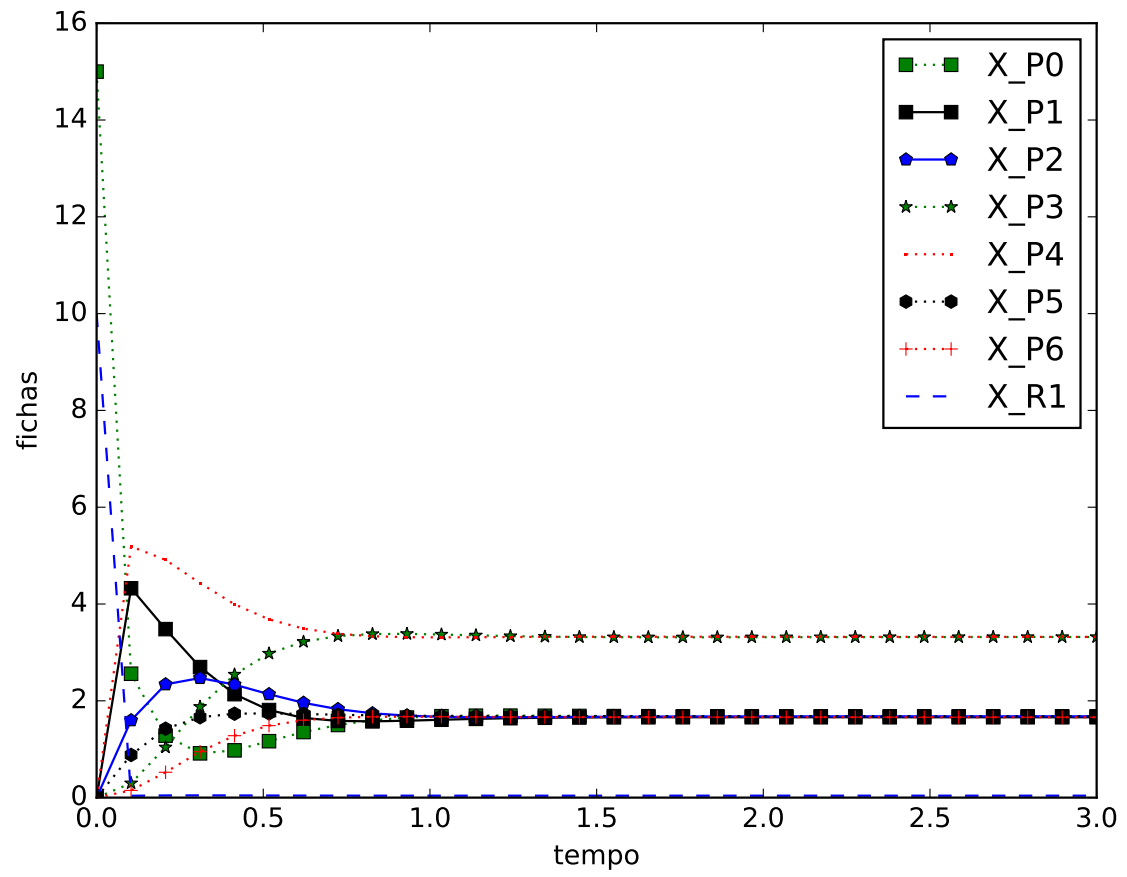

(b) Trajetórias determinísticas do SDTC mapeado para o workflow da Figura 5.7. Solução obtida em 0.158 segundos.

Figura 5.10: Comparação dos comportamentos estocástico e determinístico similar à Figura 5.9, quando I = 15 e $N R_{1}=10$.

proporcionais às probabilidades de escolha. Por outro lado, o valor de $R 1$ (recursos disponíveis) está aproximadamente em 0 , porém, apesar de serem valores muito pequenos, são diferentes nos dois tipos de soluções. 


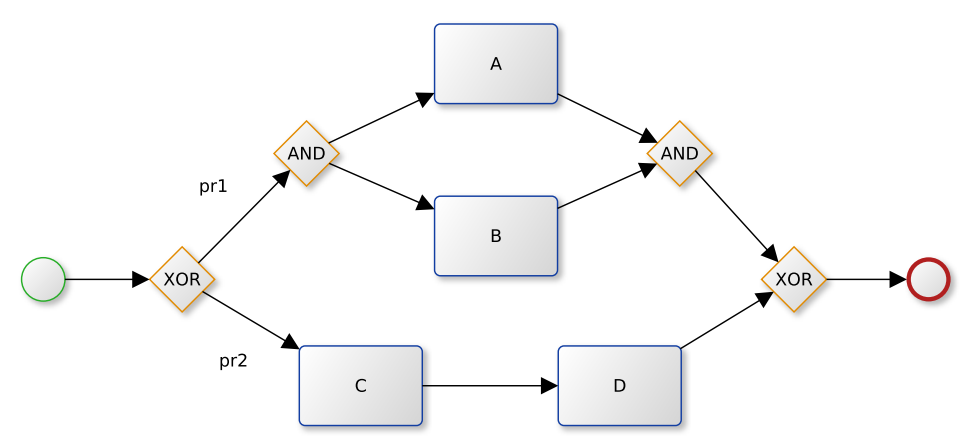

Figura 5.11: Modelo BPMN composto de estruturas básicas.

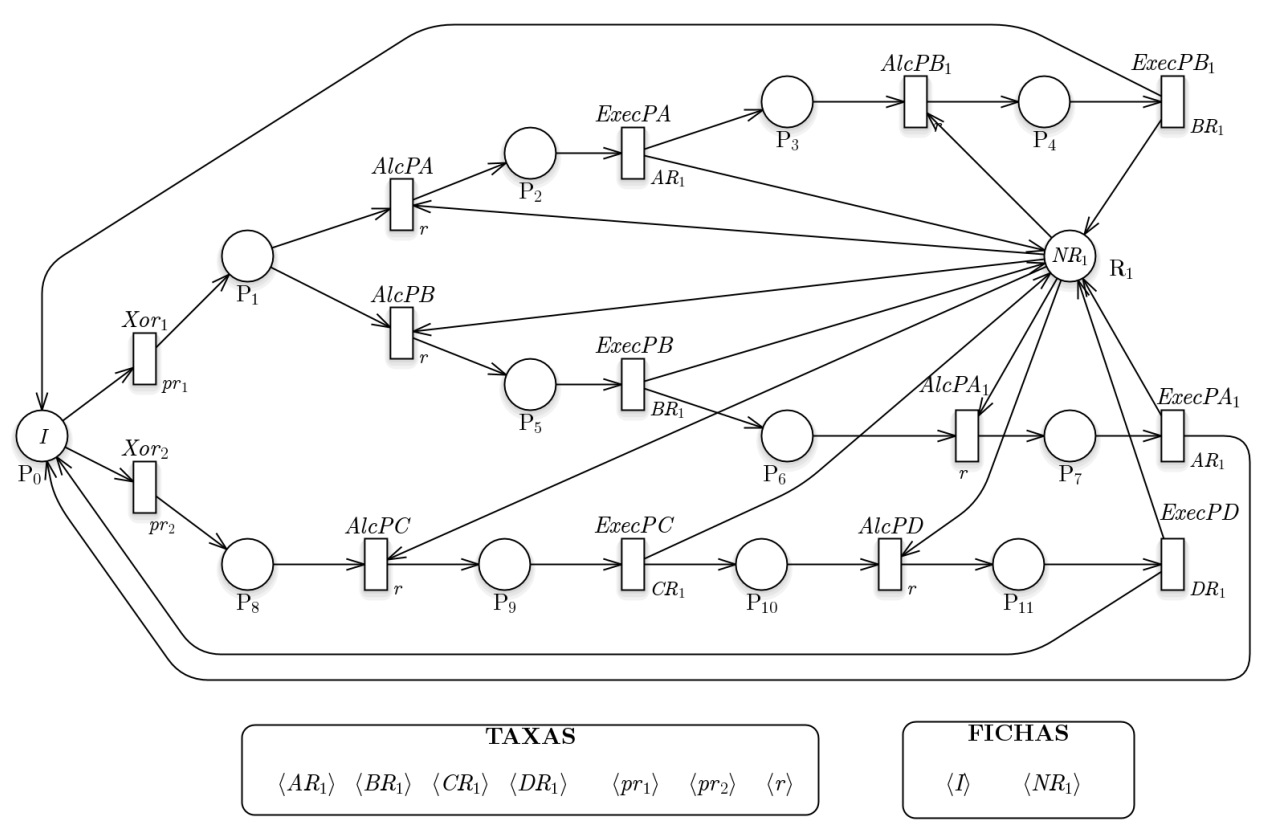

Figura 5.12: RPEG modelando o modelo de workflow mostrado na Figura 5.11.

\begin{tabular}{|c|c|c|c|}
\hline Lugar - Variável & Estocástica & Determinística & Erro (\%) \\
\hline P0 & 0.01152 & 0.01151 & 0.08681 \\
\hline P1 & 1.53902 & 1.25285 & 18.5943 \\
\hline P2 & 1.15224 & 1.15569 & 0.29942 \\
\hline P3 & 1.253 & 1.26332 & 0.82362 \\
\hline P4 & 0.57593 & 0.58289 & 1.20848 \\
\hline P5 & 0.57613 & 0.57685 & 0.12497 \\
\hline P6 & 1.25399 & 1.25775 & 0.29984 \\
\hline P7 & 1.15219 & 1.16427 & 1.04844 \\
\hline P8 & 0.50950 & 0.62639 & 22.9421 \\
\hline P9 & 1.15241 & 1.13629 & 1.3988 \\
\hline P10 & 0.439701 & 0.60659 & 37.95511 \\
\hline P11 & 0.384359 & 0.36559 & 4.88319 \\
\hline R1 & 0.00674 & 0.01842 & 173.29377 \\
\hline
\end{tabular}

Tabela 5.6: Distribuição das populações das soluções estocástica e determinística para o modelo de workflow da Figura 5.11 com 10 instâncias e 5 unidades de recurso $\left(I=10\right.$ e $\left.N R_{1}=5\right)$, cuja evolução está descrita na Figura 5.14. 


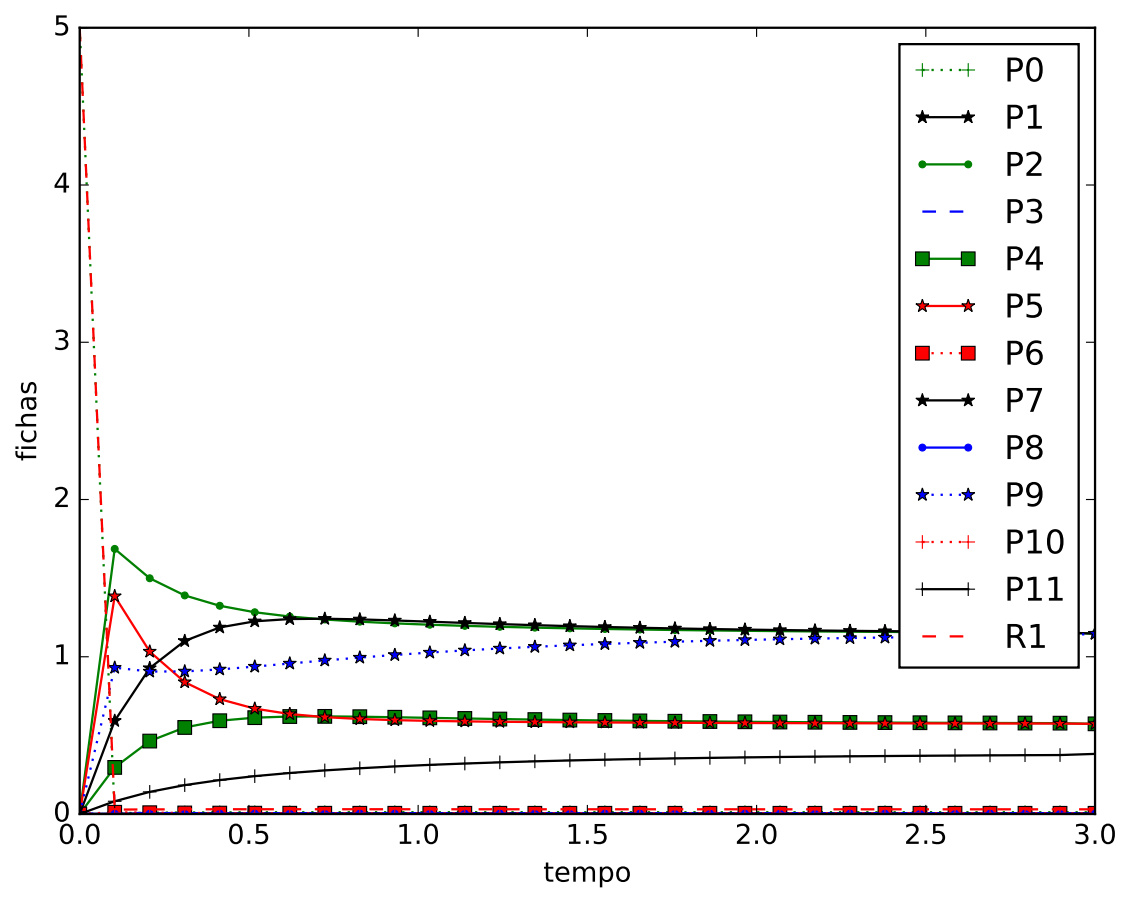

(a) Trajetórias estocásticas do valor médio de fichas nos lugares da RPEG da Figura 5.12. Solução gerada em 46.309 segundos.

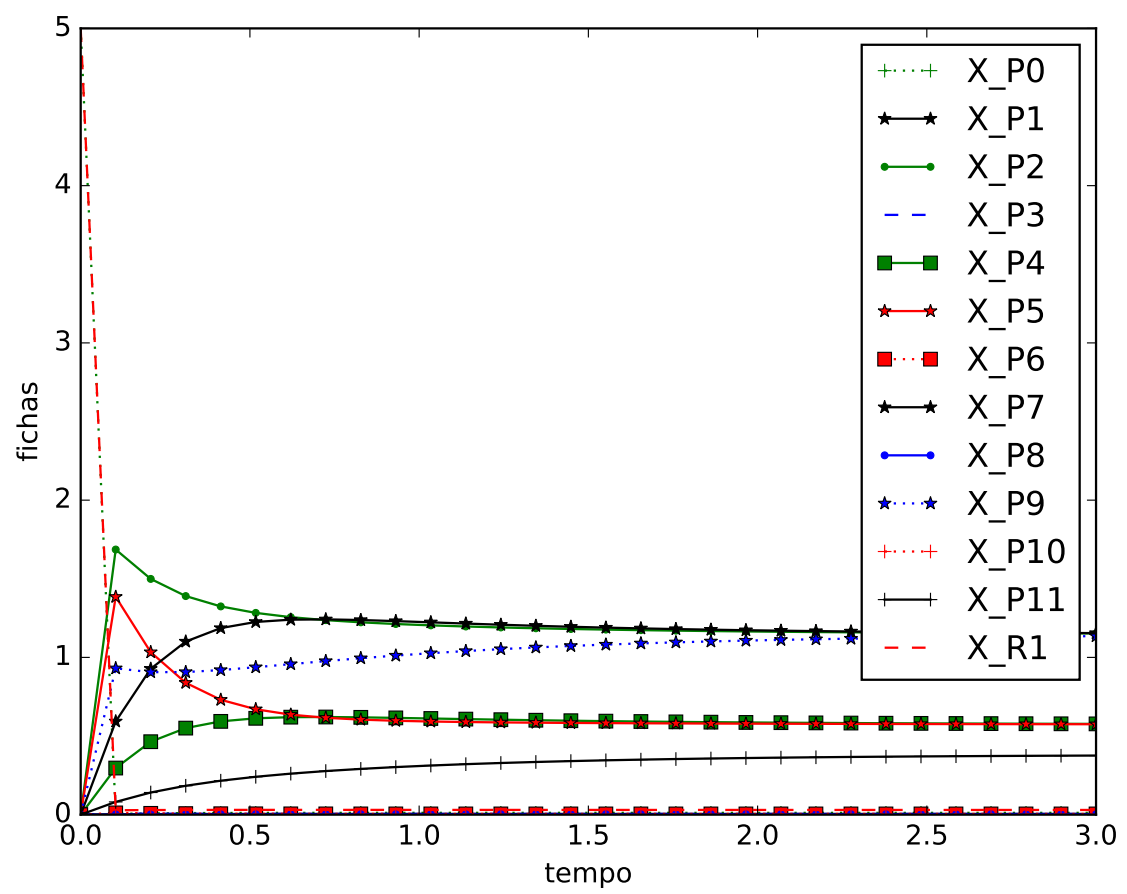

(b) Trajetórias determinísticas do SDTC mapeado para o workflow da Figura 5.11. Solução obtida em 0.163 segundos.

Figura 5.13: Comparação dos comportamentos estocástico e determinístico do workflow da Figura 5.11 para $I=5$ e $N R 1=5$. Em (b) temos que $X_{R 1}(t)=N R_{1}-\left(X_{P 2}(t)+X_{P 4}(t)+X_{P 5}(t)+X_{P 7}(t)+X_{P 9}(t)+X_{P 11}(t)\right)$. 


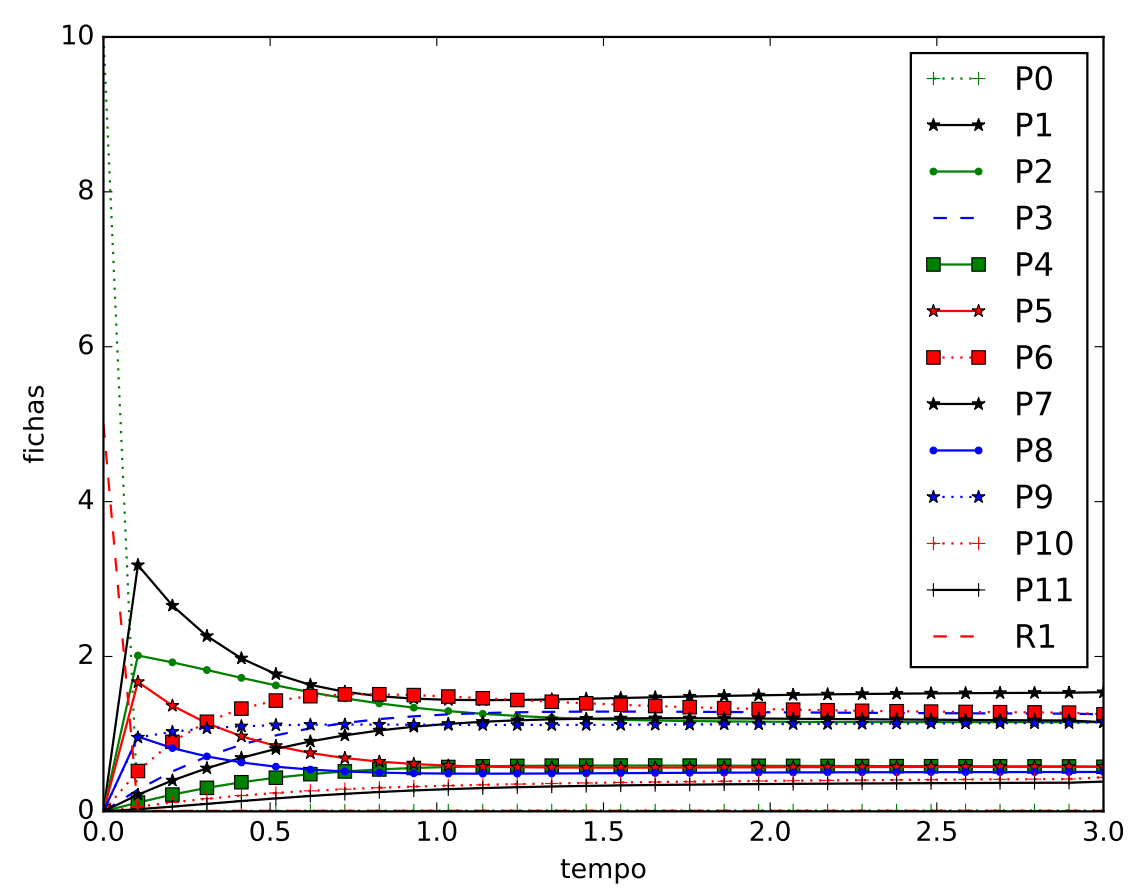

(a) Trajetórias estocásticas do valor médio de fichas nos lugares da RPEG da Figura 5.12. Solução gerada em 4345.736 segundos.

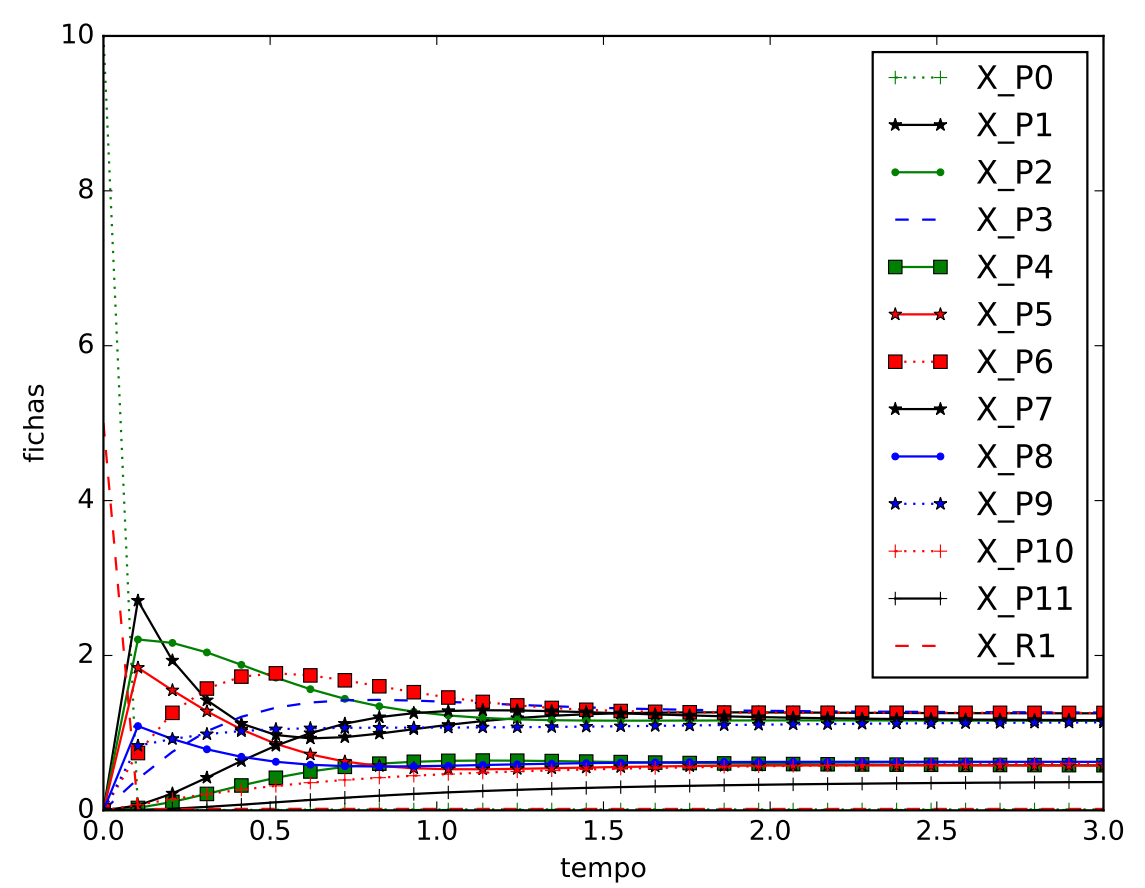

(b) Trajetórias determinísticas do SDTC mapeado para o workflow da Figura 5.11. Solução obtida em 0.164 segundos.

Figura 5.14: Comparação dos comportamentos estocástico e determinístico do workflow da Figura 5.11 para $I=10$ e NR1 $=5$. Os valores finais são descritos na Tabela 5.6. 


\subsection{Um Exemplo Mais Complexo: Workflow de Controle de Pedidos}

Nesta seção consideramos como exemplo um workflow mais complexo, mostrado na Figura 5.15 [vdA04, AD13], que descreve o controle de pedidos para uma companhia. O processamento de um pedido é dividido em duas linhas de execução paralelas principais: (i) a preparação e envio da fatura para o cliente, e (ii) o envio dos itens do pedido. Existem três tipos de trabalhadores (recursos humanos) envolvidos no processamento: os genéricos (lugar employees), os de finanças (lugar finance) e os de logística (lugar logistics). Cada um desses tipos realiza atividades específicas no processamento dos pedidos. Quando uma atividade precisa ser executada, primeiro é alocado um recurso humano do tipo correspondente à área da atividade (seta tracejada que entra na transição), o tipo de recurso é indicado pelo sufixo do lugar que segue à transição (“_E” para employees, "_F" para finance e "_L" para logistics). Depois que a atividade é executada, o recurso é desalocado (seta tracejada que sai da transição, e o recurso é indicado pelo sufixo do lugar de entrada).

O lugar start corresponde aos pedidos feitos. Um pedido pode ser registrado por qualquer tipo de recurso humano da companhia. Depois do registro, são executadas as atividades de verificação do estoque e de envio da fatura (nos lugares checking e billing respectivamente). Por um lado, os recursos em logistics estão encarregados de verificar a disponibilidade do que foi pedido (lugar checking_L e transição check_availability). Após a verificação (checked), há três continuações possíveis: (i) os itens do pedido estão em estoque (in_stock) e então o pedido é enviado (shipping, shipping_L, ship_goods e shipped); (ii) os itens do pedido estão esgotados e serão encomendados para a reposição do estoque (reorder); ou (iii) os itens do pedido estão esgotados e não serão repostos do_not_order). No caso (ii), na sequência é feita ainda a reposição (replenish, replenish_L e replenishing) e atualização dos dados do estoque (updating e update), para que se possa retomar o processamento do pedido.

Por outro lado, a preparação e cobrança da fatura, designada aos recursos em finance, consistem em enviar a fatura (billing_F, send_bill_F e billed) e receber o pagamento (receive_payment e payed). Caso não tenha sido recebido o pagamento, uma nova fatura pode ser enviada (reminder). Uma vez que o pedido foi pago e os seus itens já foram enviados, qualquer recurso pode concluir e arquivar o pedido (archiving_X, archive_X e archived, onde “_X” é um tipo de recurso).

O Código 5.1 mostra a implementação em Python das ODEs que representam a aproximação determinística para a rede de Petri da Figura 5.15. Os resultados obtidos para a solução Markoviana e a solução determinística desse modelo de workflow são mostrados na Tabela 5.7. Esses resultados foram obtidos considerando-se na análise 6 instâncias do workflow e 6 unidades de cada um dos três tipos de recursos humanos. A solução estocástica foi obtida em 78.602 segundos e a determinística em 0.168 segundos. Para alguns poucos lugares da rede (checking_L ou payed) os resultados das duas soluções são muito diferentes, mas para os demais os resultados são consistentes. Uma possível causa para as diferenças é que o modelo de workflow não cumpre todas as restrições descritas neste trabalho (sobre modelos bem-formados) para se obter uma aproximação determinística. Na rede de Petri, o paralelismo e a escolha não estão claramente definidos por estruturas de disjunção e junção, fazendo com que a modelagem não seja exatamente igual à proposta neste trabalho. Na modelo em rede de Petri, também existem atividades que não são executadas pelos recursos disponíveis. No sistema determinístico, essas atividades foram modeladas com taxas de execução independentes, que podem causar problemas na aproximação. 


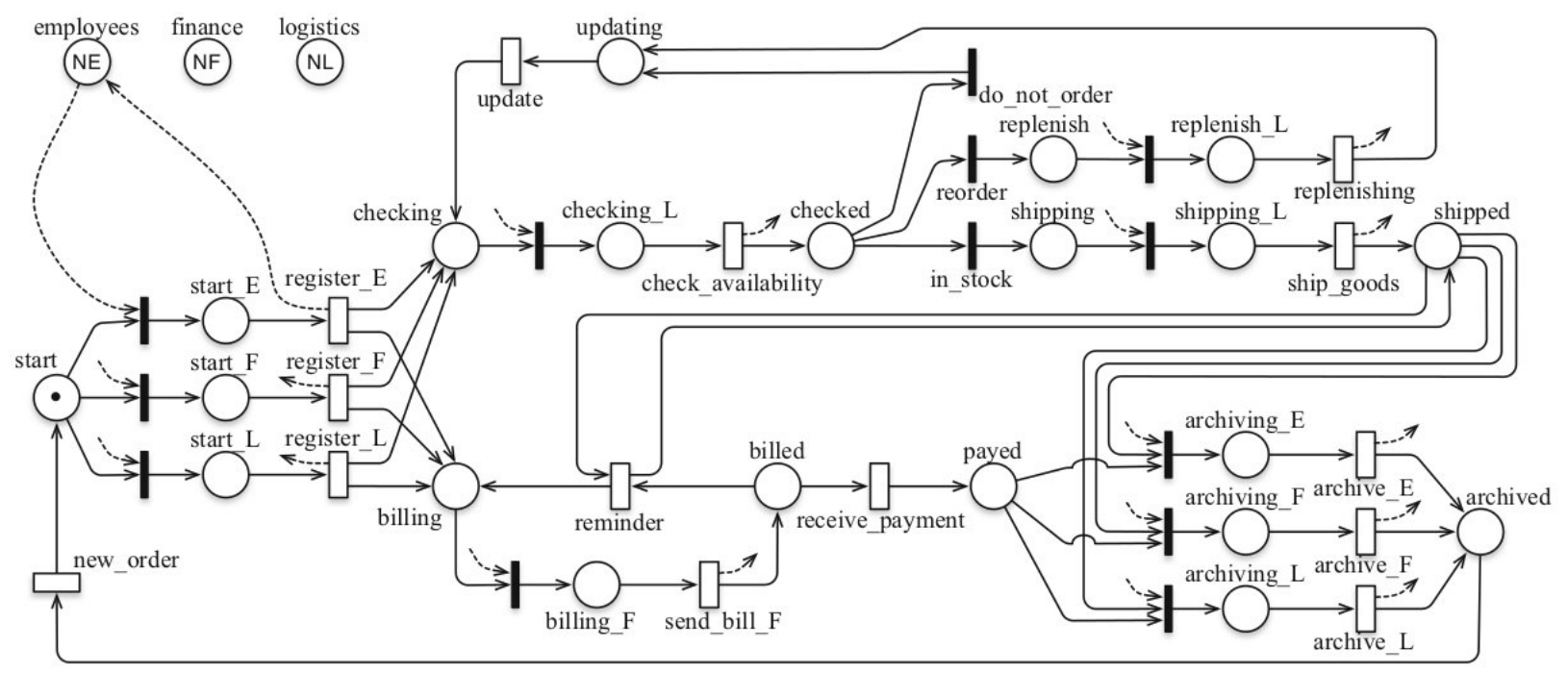

Figura 5.15: RPEG do um workflow de controle de pedidos (Extraído de [AD13]).

1 def $\operatorname{odes}(\mathrm{y}, \mathrm{t})$ :

2 \# y: vetor que representa o numero de entidades por estado (lugares da RPEG, sem os recursos) na seguinte ordem: \{start, start_E, start_F, start_L, checking, billing, updating, checking_L, checked, replenish, shipping, replenish_L, shipping_L, shipped, billing_F, billed, payed, archiving_E, archiving_F, archiving_L, archived\}

3

4

5

6

7

8

9

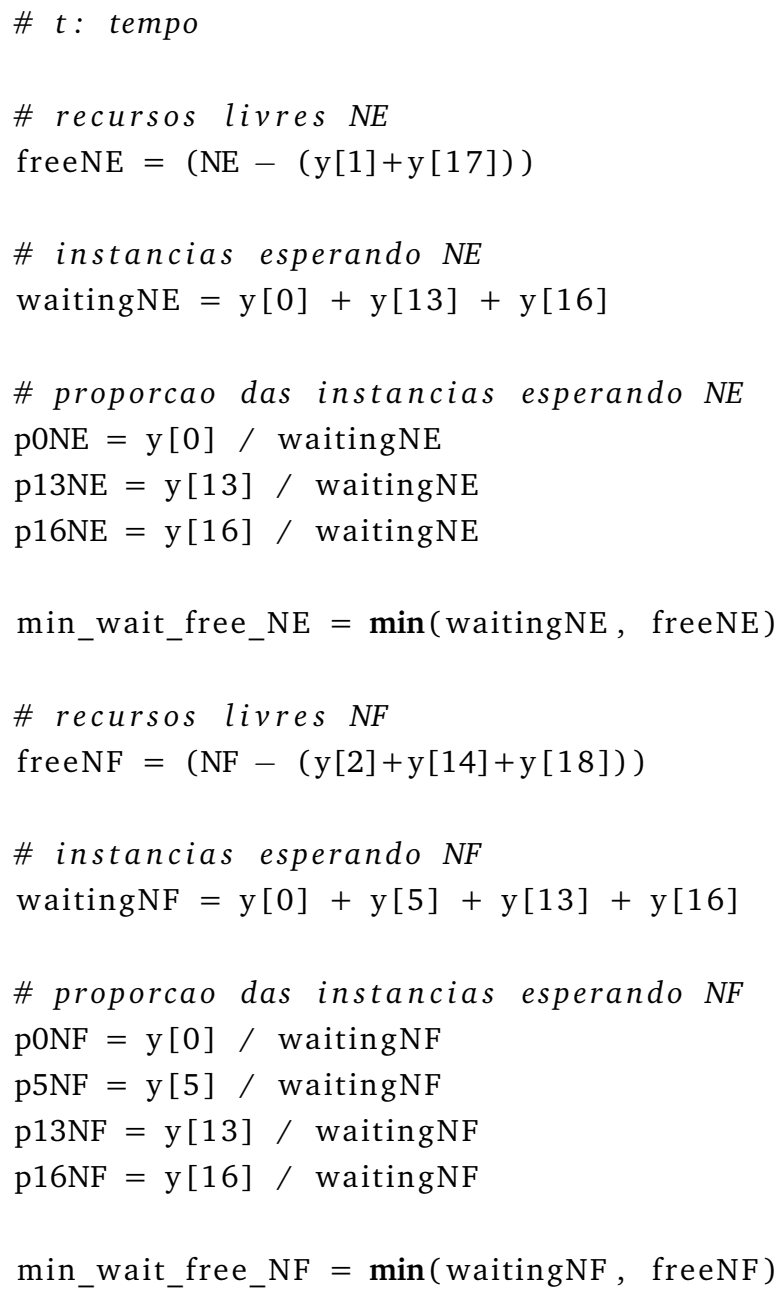


$33 \quad$ \# recursos livres $N L$

34 freeNL $=(\mathrm{NL}-(\mathrm{y}[3]+\mathrm{y}[7]+\mathrm{y}[11]+\mathrm{y}[12]+\mathrm{y}[19]))$

\# instancias esperando NL

waitingNL $=y[0]+y[4]+y[9]+y[10]+y[13]+y[16]$

\# proporcao das instancias esperando $N L$

p0NL $=\mathrm{y}[0] /$ waitingNL

$\mathrm{p} 4 \mathrm{NL}=\mathrm{y}[4] /$ waitingNL

p9NL $=y[9] /$ waitingNL

p10NL $=y[10] /$ waitingNL

p13NL $=y[13] /$ waitingNL

$\mathrm{p} 16 \mathrm{NL}=\mathrm{y}[16] /$ waitingNL

min_wait_free_NL $=\min ($ waitingNL, freeNL $)$

\# ODEs

50 fo $=-$ all $*$ pONE $*$ min_wait_free_NE - allR $*$ p0NF $*$ min_wait_free_NF - allR $*$ p0NL* min_wait_free_NL + all $*$ y[20]

$\mathrm{f} 1=-2 *$ execRegisterE $* \mathrm{y}[1]+\mathrm{allR} * \mathrm{p} 0 \mathrm{NE} * \mathrm{~min}$-wait_free_NE

$\mathrm{f} 2=-2 * \operatorname{execRegisterF} * \mathrm{y}[2]+\mathrm{allR} * \mathrm{pONF} *$ min_wait_free_NF

$\mathrm{f} 3=-2 * \operatorname{execRegisterL} * \mathrm{y}[3]+\operatorname{allR} * \mathrm{p} 0 \mathrm{NL} *$ min_wait_free_NL

$\mathrm{f} 4=-\mathrm{allR} * \mathrm{p} 4 \mathrm{NL} * \mathrm{~min} \_$wait_free_NL + execRegisterE $* \mathrm{y}[1]+$ execRegisterF $* \mathrm{y}[2]+$ execRegister $\mathrm{L} * \mathrm{y}[3]+\operatorname{allR} * \mathrm{y}[6]$

$\mathrm{f} 5=-\mathrm{allR} * \mathrm{p} 5 \mathrm{NF} * \mathrm{~min} \_\mathrm{wait}$ free_NF + execRegisterE $* \mathrm{y}[1]+$ execRegisterF $* \mathrm{y}[2]+$ execRegister $\mathrm{L} * \mathrm{y}[3]+\mathrm{a} 11 \mathrm{R} * \mathrm{y}[13]+\mathrm{allR} * \mathrm{y}[15]$

$\mathrm{f} 6=-\mathrm{allR} * \mathrm{y}[6]+\mathrm{Xor} 3 * \mathrm{y}[8]+$ execReplenishing $* \mathrm{y}[11]$

$\mathrm{f} 7=-$ execCheck_availability $* \mathrm{y}[7]+$ all $* \mathrm{p} 4 \mathrm{NL} * \mathrm{~min}$-wait_free_NL

$\mathrm{f} 8=-\mathrm{Xor} 1 * \mathrm{y}[8]-\mathrm{Xor} 2 * \mathrm{y}[8]-\mathrm{Xor} 3 * \mathrm{y}[8]+$ execCheck_availability $* \mathrm{y}[7]$

f9 $=-$ all $\mathrm{R} * \mathrm{p} 9 \mathrm{NL} * \mathrm{~min} \_$wait_free_NL + Xor $2 * \mathrm{y}[8]$

$\mathrm{f} 10=-\mathrm{allR} * \mathrm{p} 10 \mathrm{NL} * \mathrm{~min} \_$wait_free_NL + Xor $1 * \mathrm{y}[8]$

$\mathrm{f} 11=-$ execReplenishing $* \mathrm{y}[11]+$ all $\mathrm{R} * \mathrm{p} 9 \mathrm{NL} * \mathrm{~min}$ wait_free_NL

$\mathrm{f} 12=-$ execShip_goods $* \mathrm{y}[12]+$ all $\mathrm{R} * \mathrm{p} 10 \mathrm{NL} * \mathrm{~min}$-wait_free_NL

$\mathrm{f} 13=-\mathrm{allR} * \mathrm{y}[13]-\mathrm{allR} * \mathrm{p} 13 \mathrm{NE} * \mathrm{~min} \_w a i t \_f r e e \_\mathrm{NE}-$ all $\mathrm{R} * \mathrm{p} 13 \mathrm{NF} * \mathrm{~min} \_$wait_free_NF allR $* \mathrm{p} 13 \mathrm{NL} * \mathrm{~min} \_w a i t \_f r e e \_N L+$ execShip_goods $* \mathrm{y}[12]+\operatorname{allR} * \mathrm{y}[15]$

$64 \mathrm{f} 14=-\operatorname{execSend}$ bill_F $* \mathrm{y}[14]+$ all $\mathrm{R} * \mathrm{p} 5 \mathrm{NF} * \mathrm{~min}$ _wait_free_NF

$65 \mathrm{f} 15=-\mathrm{allR} * \mathrm{y}[15]-\mathrm{allR} * \mathrm{y}[15]-\mathrm{allR} * \mathrm{y}[15]+\operatorname{execSend}$ bill_F $* \mathrm{y}[14]$

$66 \mathrm{f} 16=-\mathrm{allR} * \mathrm{p} 16 \mathrm{NE} * \mathrm{~min} \_$wait_free_NE - all $\mathrm{R} * \mathrm{p} 16 \mathrm{NF} * \mathrm{~min}$ wait_free_NF - allR $* \mathrm{p} 16 \mathrm{NL} *$ min_wait_free_NL + all $\mathrm{R} * \mathrm{y}[15]$

$67 \mathrm{f} 17=-\operatorname{execArchivingE} * \mathrm{y}[17]+\mathrm{a} 11 \mathrm{R} * \mathrm{p} 13 \mathrm{NE} * \mathrm{~min} \_$wait_free_NE + all $\mathrm{R} * \mathrm{p} 16 \mathrm{NE} *$ min_wait_free_NE

$68 \mathrm{f} 18=-$ execArchiving $\mathrm{F} * \mathrm{y}[18]+\mathrm{a} 11 \mathrm{R} * \mathrm{p} 13 \mathrm{NF} * \mathrm{~min} \_$wait_free_NF + all $\mathrm{R} * \mathrm{p} 16 \mathrm{NF} *$ min_wait_free_NF

$69 \mathrm{f} 19=-\operatorname{execArchiving} \mathrm{L} * \mathrm{y}[19]+\mathrm{allR} * \mathrm{p} 13 \mathrm{NL} * \mathrm{~min} \_$wait_free_NL + all $\mathrm{R} * \mathrm{p} 16 \mathrm{NL} *$ min_wait_free_NL

$70 \mathrm{f} 20=-\operatorname{allR} * \mathrm{y}[20]+$ execArchivingE $* \mathrm{y}[17]+\operatorname{execArchiving} \mathrm{F} * \mathrm{y}[18]+$ execArchiving $\mathrm{L} * \mathrm{y}[19]$

71

72 return $[\mathrm{f} 0, \mathrm{f} 1, \mathrm{f} 2, \mathrm{f} 3, \mathrm{f} 4, \mathrm{f} 5, \mathrm{f} 6, \mathrm{f} 7, \mathrm{f} 8, \mathrm{f} 9, \mathrm{f} 10, \mathrm{f} 11, \mathrm{f} 12, \mathrm{f} 13, \mathrm{f} 14, \mathrm{f} 15$, $\mathrm{f} 16, \mathrm{f} 17, \mathrm{f} 18, \mathrm{f} 19, \mathrm{f} 20]$

Código 5.1: ODEs do modelo em 5.15 


\begin{tabular}{|c|c|c|c|}
\hline \multirow{2}{*}{$\begin{array}{c}\text { Lugar } \\
\text { (Variável) }\end{array}$} & \multicolumn{3}{|c|}{ População Segundo a Solução } \\
\cline { 2 - 4 } & Estocástica & Determinística & Erro (\%) \\
\hline employees & 5.54099 & 5.56396 & 0.41455 \\
\hline finance & 4.09023 & 3.87921 & 5.15912 \\
\hline logistics & 1.48666 & 2.59692 & 74.6815 \\
\hline start & 0.00184 & 0.00231 & 25.54348 \\
\hline start_E & 0.22951 & 0.14451 & 37.03542 \\
\hline start_F & 0.45903 & 0.28898 & 37.0455 \\
\hline start_L & 0.91786 & 0.57827 & 36.99802 \\
\hline checking & 0.00918 & 0.00579 & 36.9281 \\
\hline checking_L & 1.52997 & 0.96719 & 36.78373 \\
\hline checked & 0.00918 & 0.00580 & 36.81917 \\
\hline updating & 0.00367 & 0.00233 & 36.51226 \\
\hline shipping & 0.00551 & 0.00348 & 36.8421 \\
\hline shipping_L & 0.68849 & 0.43589 & 36.68899 \\
\hline shipped & 0.07289 & 0.00149 & 97.95582 \\
\hline replenish & 0.00184 & 0.00116 & 36.95652 \\
\hline replenish_LL & 0.45899 & 0.29150 & 36.49099 \\
\hline billing & 0.00595 & 0.00746 & 25.37815 \\
\hline billed & 0.00551 & 0.00249 & 54.80944 \\
\hline billing_F & 0.99173 & 1.24591 & 25.62996 \\
\hline payed & 1.77654 & 0.00083 & 99.95328 \\
\hline archive_E & 0.22950 & 0.29154 & 27.03268 \\
\hline archive_F & 0.45901 & 0.58589 & 27.64209 \\
\hline archive_L & 0.91801 & 1.13022 & 23.1163 \\
\hline archived & 0.00551 & 0.00694 & 25.95281 \\
\hline
\end{tabular}

Tabela 5.7: Soluções estocástica e determinística para a RPEG descrita na Figura 5.15

\subsection{Considerações sobre os resultados}

Neste capítulo foram apresentadas funções de indexação que permitem o cálculo de medidas de desempenho, as quais são elaboradas e formuladas sobre as variáveis das ODEs, que é a aproximação determinística do modelo de workflow. Foram mostrados experimentos e resultados comparando trajetórias, que descrevem a evolução das variáveis do sistema, do modelo estocástico e do modelo contínuo. Existem cenários em que alguns resultados para certos tipos de estados parecem divergir mais quanto menor for o tamanho do sistema. Todavia, no geral, as aproximações determinísticas são bem próximas aos valores obtidos por meio de técnicas Markovianas, já que temos erros porcentuais que geralmente são menores que $20 \%$, a propósito, para estruturas de sequência de atividades e paralelismo, esses valores são muito menores ainda. Porém, no último exemplo, na Tabela 5.7, os erros porcentuais incrementaram, sendo a maioria menor que 40\%. É necessário evidenciar o fato que foram comparados valores para populações muito pequenos, os quais mostram grandes erros porcentuais, algumas vezes maiores que $100 \%$. 


\section{Capítulo 6}

\section{Conclusões}

\subsection{Considerações Finais}

No presente trabalho foi desenvolvido um novo método de análise preditiva de desempenho para workflows com numerosas instâncias executadas paralelamente em uma quantidade limitada de recursos. Este método demonstrou superar o problema da explosão de estados, modelando o comportamento dos sistemas com espaços de estados contínuos, tendo como limite o número de variáveis geradas nas ODEs.

Tomamos como base um subconjunto dos modelos de workflow que podem ser descritos com a notação BPMN, os denominados workflows bem-definidos. Além disso, consideramos que esses modelos podem ser complementados com a descrição dos recursos disponíveis para a execução e as especificações de requisitos de recursos para cada uma das atividades que compõem o workflow. A partir disso, foi descrito um método de mapeamento de um modelo de workflow em um conjunto de equações diferenciais ordinárias (ODEs), que descrevem um sistema determinístico de tempo contínuo (SDTC) que aproxima o comportamento do sistema inicial. No processo de mapeamento, foi utilizado como modelo intermediário uma cadeia de Markov de tempo contínuo de população (CMTCP), que é um modelo Markoviano baseado nos estados em que uma instância de workflow pode estar durante sua execução. Com as CMTCP, já temos uma visão macroscópica do sistema, pois foca-se nos estados possíveis das entidades. Contudo, ele ainda permite um acompanhamento individual das entidades para estabelecer seus estados, utilizando estruturas de dados de indexação.

Dentre as estruturas básicas de construção de modelos de workflow (i.e., sequência, escolha e paralelismo), a que se mostrou mais complexa de ser mapeada foi o paralelismo. O seu procedimento de mapeamento é de complexidade $O(n !)$, pois são modeladas todas as possíveis permutações dos $n$ fluxos paralelos (criados por um componente $A N D$-split). Desse modo, quanto maior o número de fluxos paralelos, muitos mais estados possíveis tem uma instância do workflow.

O método de mapeamento do modelo de workflow em um SDTC segue uma estratégia bem definida, o que possibilita a automação do método. Então, é possível definir um procedimento que tenha como entrada um modelo BPMN do workflow, assim como suas especificações de recursos e os requisitos das atividades, e produza um SDTC descrito por um conjunto de ODEs.

Por meio da solução das ODEs, é possível calcular medidas de desempenho. Para isso, é necessário fazer uma indexação das variáveis que constam nas ODEs. Na etapa de mapeamento do modelo de workflow, para cada componente são criados alguns estados e transições, os quais descrevem o seu comportamento, e esses estados e transições fazem parte da solução determinística. Portanto, para obter um índice de desempenho, fazemos um cálculo sobre variáveis específicas, referentes 
aos estados de interesse para o índice. O exemplo mais notório é o caso do conjunto de estados e transições resultante do mapeamento de uma atividade do workflow. Os índices de desempenho da atividade são determinados por esse conjunto de estados e transições.

Os índices de desempenho são computados em tempos razoáveis e com baixa demanda de recursos computacionais, já que não são geradas grandes quantidades de dados. Dessa forma, o método proposto oferece maior flexibilidade na realização de experimentos com variação de valores para os parâmetros do workflow, o que é particularmente interessante para os parâmetros que afetam diretamente os índices de desempenho.

Uma das maiores dificuldades que se enfrentou neste trabalho foi a validação experimental dos resultados. Isso se deve ao fato de que, para modelos de workflow onde são executadas várias dezenas de instâncias, não conseguimos obter uma solução estocástica, impossibilitando a comparação com as soluções determinísticas. Para modelos relativamente simples, as soluções estocásticas podem ser obtidas para sistemas com algumas poucas centenas de instâncias.

Todavia, o método proposto neste trabalho foi definido formalmente com base em teoremas já bem desenvolvidos, verificando-se, ainda, as condições do modelo estocástico do workflow que garantem a existência de uma aproximação por um sistema determinístico. Então, os resultados obtidos por meio do método são promissores, ainda que sejam aproximações. E nas comparações experimentais realizadas com workflows com pequenos números de instâncias (as únicas possíveis de serem realizadas também com modelos estocásticos), as aproximações por sistema determinístico se mostraram suficientemente boas. Cabe mencionar, que a aproximação determinística é aplicável a qualquer sistema que cumpra as restrições para ter um limite determinístico.

\subsection{Sugestões para Pesquisas Futuras}

\section{Modelagem Analítica de Workflows e Recursos Mais Sofisticados}

A estrutura do modelo de workflow, assim como suas especificações de recursos e requisitos, foram restringidas de tal forma que seja garantida a existência da aproximação determinística. Entretanto, um estudo mais minucioso de outras características relacionadas à estrutura do workflow, às políticas de acesso aos recursos, às quantidades e capacidades de trabalho funcionais, à alocação de recursos sob demanda (e outros) pode resultar em novos tipos de processos, cujos comportamentos talvez possam ser aproximados deterministicamente.

\section{Medição do Erro da Aproximação Determinística}

Tendo em vista a dificuldade de comparar resultados obtidos de formas estocástica e determinística, surge também o problema de medir o erro das aproximações. Em [BHLM13] é estabelecido um limite para medir o erro da solução determinística com relação à solução estocástica. Porém, na prática, esse ainda é um problema em aberto. Assim, futuras pesquisas podem ser direcionadas à medição do erro da aproximação determinística do desempenho dos workflows.

\section{Linguagens de Consulta de Índices de Desempenho}

As técnicas Markovianas têm sido amplamente estudadas e utilizadas na análise de desempenho. Assim, foram criadas linguagens de consulta de índices de desempenho, para serem utilizadas por usuários finais. Com essas linguagens, os usuários são capazes de definir funções de indexação para calcular medidas de desempenho de interesse específico. Então, baseado na aproximação determinística gerada neste trabalho, pode ser interessante definir linguagens de consulta de índices 
de desempenho flexíveis e amigáveis para os usuários dos workflows.

\section{Simulação do Comportamento de um Conjunto Determinado de Instâncias do Workflow}

Uma visão microscópica do sistema acarreta a perda de detalhes das instâncias individuais. Em [LMM07] é apresentado um resultado de simulação, que permite o acompanhamento da evolução de um grupo específico de entidades dentro de um grande sistema de objetos que interagem entre si. Esse resultado foi obtido para sistemas determinísticos de tempo discreto. Então, essa simulação pode ser estudada para também ser aplicada em SDTCs que descrevem workflows, possibilitando assim avaliar o desempenho de um conjunto determinado de instâncias do workflow. 
CONCLUSÕES 


\section{Referências Bibliográficas}

[AD13] Elvio Gilberto Amparore e Susanna Donatelli. Improving and assessing the efficiency of the MC4CSLTA model checker. Lecture Notes in Computer Science, páginas 206-220, 2013. xii, 27, 70, 71

[Atk99] Roger Atkinson. Project management: cost, time and quality, two best guesses and a phenomenon, its time to accept other success criteria. International journal of project management, 17(6):337-342, 1999. 3

[Bal07] Gianfranco Balbo. Introduction to generalized stochastic petri nets. Em International School on Formal Methods for the Design of Computer, Communication and Software Systems, páginas 83-131. Springer, 2007. 22, 23, 25

$\left[\mathrm{BBC}^{+}\right.$09] Soheib Baarir, Marco Beccuti, Davide Cerotti, Massimiliano De Pierro, Susanna Donatelli e Giuliana Franceschinis. The greatspn tool: Recent enhancements. SIGMETRICS Perform. Eval. Rev., 36(4):4-9, Março 2009. 22, 27

[BC14] Kelly Rosa Braghetto e Daniel Cordeiro. Introdução à modelagem e execução de workflows científicos. Atualizações em Informática. 1ed. Porto Alegre: SBC, páginas 1-40, 2014. 7, 8

[BCFH11] Rena Bakhshi, Lucia Cloth, Wan Fokkink e Boudewijn R. Haverkort. Mean-field framework for performance evaluation of pushpull gossip protocols. Performance Evaluation, 68(2):157-179, 2011. 42

[BGT08] A. Bobbio, M. Gribaudo e M. Telek. Analysis of Large Scale Interacting Systems by Mean Field Method. 2008 Fifth International Conference on Quantitative Evaluation of Systems, páginas 1-10, 2008. 42

[BHHK03] Christel Baier, Boudewijn Haverkort, Holger Hermanns e J-P Katoen. Modelchecking algorithms for continuous-time markov chains. IEEE Transactions on software engineering, 29(6):524-541, 2003. 40

[BHLM13] Luca Bortolussi, Jane Hillston, Diego Latella e Mieke Massink. Continuous approximation of collective system behaviour: A tutorial. Performance Evaluation, 70(5):317-349, 05 2013. 41, 42, 44, 46, 76

[BLB08] Michel Benaim e Jean-Yves Le Boudec. A class of mean field interaction models for computer and communication systems. Performance Evaluation, 65(11):823-838, 2008. 42

[BMP07] Charles Bordenave, David McDonald e Alexandre Proutiere. A particle system in interaction with a rapidly varying environment: Mean field limits and applications. página $31,2007.42$

[Bra11] Kelly Rosa Braghetto. Técnicas de modelagem para a análise de desempenho de processos de negócio. Tese de Doutorado, Universidade de Sao Paulo, 2011. 14, 21, 22 
[BW90] Rabi N. Bhattacharya e Edward C. Waymire. Stochastic processes with applications, volume 61. Siam, 1990. 2, 18, 19

[CFGR95] Giovanni Chiola, Giuliana Franceschinis, Rossano Gaeta e Marina Ribaudo. Greatspn 1.7: graphical editor and analyzer for timed and stochastic petri nets. Performance evaluation, 24(1):47-68, 1995. 22, 27

[CMBC93] G. Chiola, M. Marsan, G. Balbo e G. Conte. Generalized stochastic petri nets: A definition at the net level and its implications. IEEE Transactions on Software Engineering, 19(2):89-107, 1993. 24

[CR83] J. Coolahan e N. Roussopoulos. Timing requirements for time-driven systems using augmented petri nets. IEEE Transactions on Software Engineering, SE-9(5):603-616, Sep 1983. 24

[Dur12] Richard Durrett. Essentials of stochastic processes. Springer Science \& Business Media, 2012. 19

[EN94] Javier Esparza e Mogens Nielsen. Decidability issues for petri nets. BRICS Report Series, 1(8), 1994. 39

[FK09] Alexander Fronk e Britta Kehden. State space analysis of petri nets with relationalgebraic methods. Journal of Symbolic Computation, 44(1):15 - 47, 2009. 39

[FM03] Paul Fortier e Howard Michel. Computer systems performance evaluation and prediction. Digital Press, 2003. 16

[GGL12] Nicolas Gast, Bruno Gaujal e Jean-Yves Le Boudec. Mean Field for Markov Decision Processes: From Discrete to Continuous Optimization. IEEE Transactions on Automatic Control, 57(9):2266-2280, 2012. 42

[Har87] David Harel. Statecharts: A visual formalism for complex systems. Science of computer programming, 8(3):231-274, 1987. 22

[Hil14] Jane Hillston. The Benefits of Sometimes Not Being Discrete. Blog.Inf.Ed.Ac.Uk, páginas 7-22, 2014. 42

[HK94] Johannes Helbig e Peter Kelb. An obdd-representation of statecharts. Em European Design and Test Conference, 1994. EDAC, The European Conference on Design Automation. ETC European Test Conference. EUROASIC, The European Event in ASIC Design, Proceedings., páginas 142-149. IEEE, 1994. 22

[Hol95] David Hollingsworth. Workflow management coalition the workflow reference model. (1.1):1-55, Janeiro 1995. Relatório Técnico TC00-1003, Workflow Management Coalition. 1, 7

[HRS13] Monika Heiner, Christian Rohr e Martin Schwarick. MARCIE - Model Checking and Reachability Analysis Done Efficiently, páginas 389-399. Springer Berlin Heidelberg, Berlin, Heidelberg, 2013. 22

[Jai91] Raj Jain. The Art of Computer Systems Performance Analysis: Techniques for Experimental Design, Measurement, Simulation, and Modeling, volume 491. John Wiley \& Sons, New York, NY, 1991. 2, 16, 17

[Kar91] Ioannis Karatzas. Brownian motion and stochastic calculus, volume 113. Springer Science \& Business Media, 1991. 41

[Kar14] Samuel Karlin. A first course in stochastic processes. Academic press, 2014. 19 
[Kur70] Thomas G. Kurtz. Solutions of ordinary differential equations as limits of pure jump markov processes. Journal of Applied Probability, 7(01):49-58, Apr 1970. 46

[LMM07] Jean Yves Le Boudec, David McDonald e Jochen Mundinger. A generic mean field convergence result for systems of interacting objects. Proceedings - 4th International Conference on the Quantitative Evaluation of Systems, QEST 2007, páginas 3-15, 2007. 42, 44, 46, 77

[LZJ16] Guanjun Liu, Kun Zhang e Changjun Jiang. Deciding the Deadlock and Livelock in a Petri Net with a Target Marking Based on Its Basic Unfolding, páginas 98-105. Springer International Publishing, Cham, 2016. 23

[Men09] Elliott Mendelson. Introduction to mathematical logic. CRC press, 2009. 14

[Mol82] M. Molloy. Performance analysis using stochastic petri nets. IEEE Trans. Comput., 31(9):913-917, Setembro 1982. 24

[NJ00] M. Nicola e M. Jarke. Performance modeling of distributed and replicated databases. IEEE Transactions on Knowledge and Data Engineering, 12(4):645-672, 2000. 16

[OMG] OMG. Object management group. http://www.omg.org/. Último acesso em 26/10/2016. 10

[OMG11] OMG. Business Process Model and Notation (BPMN) Version 2.0, 2011. 10

[OS01] Manfred Opper e David Saad. Advanced mean field methods: Theory and practice. MIT press, 2001. 3, 41

[OW00] Manfred Opper e Ole Winther. Gaussian processes for classification: Mean-field algorithms. Neural Computation, 12(11):2655-2684, 2000. 3, 42

[Pla85] Brigitte Plateau. On the stochastic structure of parallelism and synchronization models for distributed algorithms. Em ACM SIGMETRICS Performance Evaluation Review, volume 13, páginas 147-154. ACM, 1985. 22

[R $\mathrm{R}^{+}$96] Sheldom Ross et al. Stochastic processes, volume 2. John Wiley \& Sons New York, 1996. 2,18

[RHE04] Nick Russell, Arthur H M Hofstede e David Edmond. Workflow resource patterns. Business, 3520:13-17, 2004. 13

[Ros14] Sheldom Ross. Introduction to probability models. Academic press, 2014. 20, 43, 44

[RS07] W. Rouse e A. Sage. Work, Workflow and Information Systems. Tennenbaum Institute series on enterprise systems. IOS Press, 2007. 8

[RvdATHE05] Nick Russell, W. van der Aalst, Arthur HM Ter Hofstede e David Edmond. Workflow resource patterns: Identification, representation and tool support. Em Advanced Information Systems Engineering, páginas 216-232. Springer, 2005. 8

[SM09] M. Schumann e J. Michael. Statechart based formal modeling of workflow processes. 2009 IEEE International Conference on System of Systems Engineering (SoSE), 2009. 22

[Ste94] Williams J Stewart. Introduction to the numerical solutions of Markov chains. Princeton Univ. Press, Princeton, NJ, 1994. 40 
[vdA98] W. van der Aalst. Three good reasons for using a Petri-net-based workflow management system. Information and Process Integration in Enterprises: Rethinking documents, 08:161-182, 1998. 22

[vdA04] W. van der Aalst. Business process management demystified: A tutorial on models, systems and standards for workflow management. Lectures on Concurrency and Petri Nets, páginas 1-65, 2004. 27, 70

[vdABtHK00] W. van der Aalst, A.P. Barros, A.H.M. ter Hofstede e B. Kiepuszewski. Advanced workflow patterns. Em Peter Scheuermann e Opher Etzion, editors, Cooperative Information Systems, volume 1901 of Lecture Notes in Computer Science, páginas 18-29. Springer Berlin Heidelberg, 2000. 8

[vdAtHKB03] W. van der Aalst, A. ter Hofstede, B. Kiepuszewski e A. Barros. Workflow patterns. Distributed and Parallel Databases, 14(1):5-51, 2003. 7, 8

[VDAVH04] W. Van Der Aalst e Kees Max Van Hee. Workflow management: models, methods, and systems. MIT press, 2004. 7, 9, 22

[WfM99] WfMC. Workflow management coalition terminology \& glossary. (3):1-65, Fevereiro 1999. Relatório Técnico WFMC-TC-1011, Workflow Management Coalition. 1,8

[WW97] Dirk Wodtke e Gerhard Weikum. A formal foundation for distributed workflow execution based on state charts. Em Database Theory - ICDT'97, páginas 230-246. Springer, 1997. 22 